\title{
Search for new phenomena in $p p$ collisions in final states with tau leptons, $b$-jets, and missing transverse momentum with the ATLAS detector
}

\author{
G. Aad et al. \\ (ATLAS Collaboration)
}

(Received 18 August 2021; accepted 8 September 2021; published 21 December 2021)

\begin{abstract}
A search for new phenomena in final states with hadronically decaying tau leptons, $b$-jets, and missing transverse momentum is presented. The analyzed dataset comprises $p p$ collision data at a center-of-mass energy of $\sqrt{s}=13 \mathrm{TeV}$ with an integrated luminosity of $139 \mathrm{fb}^{-1}$, delivered by the Large Hadron Collider and recorded with the ATLAS detector from 2015 to 2018. The observed data are compatible with the expected Standard Model background. The results are interpreted in simplified models for two different scenarios. The first model is based on supersymmetry and considers pair production of top squarks, each of which decays into a $b$-quark, a neutrino and a tau slepton. Each tau slepton in turn decays into a tau lepton and a nearly massless gravitino. Within this model, top-squark masses up to $1.4 \mathrm{TeV}$ can be excluded at the $95 \%$ confidence level over a wide range of tau-slepton masses. The second model considers pair production of leptoquarks with decays into third-generation leptons and quarks. Depending on the branching fraction into charged leptons, leptoquarks with masses up to around $1.25 \mathrm{TeV}$ can be excluded at the 95\% confidence level for the case of scalar leptoquarks and up to $1.8 \mathrm{TeV}(1.5 \mathrm{TeV})$ for vector leptoquarks in a Yang-Mills (minimal-coupling) scenario. In addition, model-independent upper limits are set on the cross section of processes beyond the Standard Model.
\end{abstract}

DOI: $10.1103 /$ PhysRevD.104.112005

\section{INTRODUCTION}

The Standard Model (SM) of particle physics has been verified to high precision. Despite its success, several observations have been made which have exposed the theory's shortcomings in various aspects and fostered new theoretical ideas. Supersymmetry (SUSY) [1-7] is a framework for models that extend the symmetries underlying the SM by introducing superpartners of the known bosons and fermions with the same quantum numbers but a spin difference of half a unit. These models can address the gauge hierarchy problem [8-11]. When conservation of $R$-parity [12] is assumed, the lightest supersymmetric particle is stable and may provide a candidate particle for the cold dark matter component of the Universe [13,14]. The introduction of supersymmetric partner particles can also modify the renormalization group equations in such a way that the coupling constants of the SM electromagnetic, weak and strong interactions meet at one point at some high energy scale as expected in a grand unified theory [15]. Another possible way to extend the SM is to embed the SM

*Full author list given at the end of the article.

Published by the American Physical Society under the terms of the Creative Commons Attribution 4.0 International license. Further distribution of this work must maintain attribution to the author(s) and the published article's title, journal citation, and DOI. Funded by SCOAP ${ }^{3}$. symmetry group in an overarching symmetry group, such as $\mathrm{SU}(5)$ [16] in grand unification, which gives rise to a new class of bosons that carry nonzero baryon and lepton quantum numbers and are charged under all SM gauge groups. These leptoquarks (LQ), which can be either scalar or vector bosons, appear in a variety of SM extensions [17-21] and would provide an explanation for the structural similarities of the quark and lepton sectors in the SM. Processes mediated by the exchange of leptoquarks can violate lepton-flavor universality and have been proposed as an explanation [22-28] for the deviations from the SM predictions seen by many experiments in measurements of $B$-meson decays [29-37]. Contributions arising from leptoquarks with additional couplings to the muon could also bridge the gap $[38,39]$ between the theoretical prediction for the anomalous magnetic moment of the muon $(g-2)_{\mu}$ within the SM and the experimentally measured value, which is higher by $4.2 \sigma$ [40].

In this paper, a search for physics beyond that described in the Standard Model is conducted using events with final states with one or more hadronically decaying tau leptons, one or more $b$-tagged jets and large missing transverse momentum. This is a signature that is sensitive to models in which the new particles preferentially decay into thirdgeneration SM particles. Two benchmark signal models are studied. The first model considers the production of supersymmetric partner states of the third-generation SM particles, while the second model foresees scalar 
leptoquarks that decay into third-generation SM particles. An additional interpretation, for which the analysis was not explicitly optimized, is provided for vector leptoquarks that decay into third-generation SM particles. The full run-2 dataset of proton-proton $(p p)$ collisions recorded with the ATLAS detector at the Large Hadron Collider (LHC) is analyzed. This dataset corresponds to an integrated luminosity of $139 \mathrm{fb}^{-1}$, taken from 2015 through 2018, at a center-of-mass energy of $\sqrt{s}=13 \mathrm{TeV}$.

The investigated SUSY signal model is motivated by gauge-mediated SUSY breaking (GMSB) [41-43] and natural gauge mediation [44]. In this $R$-parity-conserving scenario, only three SUSY particles are assumed to be sufficiently light to be relevant: the lighter scalar partner of the top quark $\tilde{t}$ (top squark or stop), the lighter scalar partner of the tau lepton $\tilde{\tau}$ (tau slepton or stau), and the spin-3/2 partner of the graviton, the gravitino $\tilde{G}$. The top squark is assumed to be the lightest squark $[45,46]$ and to be directly pair-produced through the strong interaction. The gravitino is assumed to be almost massless, making it the lightest SUSY particle (LSP) in this scenario. The search strategy is optimized using a simplified model [47-49] with this limited SUSY particle content, the model parameters being the scalar-fermion masses $m(\tilde{t})$ and $m(\tilde{\tau})$. The decay chain is illustrated in the left diagram of Fig. 1: a three-body decay proceeding through an off shell chargino $\tilde{t} \rightarrow b \tilde{\tau} \nu_{\tau}$ followed by $\tilde{\tau} \rightarrow \tau \tilde{G}$. This model is referred to as the "stop-stau" signal model in the following. When the LSP is the gravitino, direct decays of SUSY particles into the gravitino LSP (plus a SM particle) are very unlikely due to its weak coupling, except for the next-to-lightest supersymmetric particle, which in $R$-parity-conserving scenarios has no other option than to decay into the gravitino LSP. Other SUSY models which instead assume the lightest neutralino $\tilde{\chi}_{1}^{0}$ to be the LSP are not studied here, as this would favor a high branching fraction of $\tilde{t} \rightarrow t \tilde{\chi}_{1}^{0}$; this case has been studied elsewhere by the ATLAS Collaboration [50-52] and by the CMS Collaboration [53-56].

Previous searches by the ATLAS Collaboration for signals in this model used $20 \mathrm{fb}^{-1}$ of $\sqrt{s}=8 \mathrm{TeV}$ data taken in run 1 [57] and $36.1 \mathrm{fb}^{-1}$ of $\sqrt{s}=13 \mathrm{TeV}$ data taken in run 2 of the LHC [58]. No significant excess was observed in either of these searches, and thus limits were set on the masses of the top squark and tau slepton. These limits exclude top-squark masses of up to $1.16 \mathrm{TeV}$ and tauslepton masses of up to $1.0 \mathrm{TeV}$ at the $95 \%$ confidence level. The CMS Collaboration has published a related search in a simplified model with pair production of top squarks, which are also assumed to decay via tau sleptons or tau sneutrinos, but where the LSP is the lightest neutralino $\tilde{\chi}_{1}^{0}$ instead of the gravitino [59]. This search is based on an integrated luminosity of $77.2 \mathrm{fb}^{-1}$ and sets exclusion limits at the 95\% confidence level on the top-squark mass of up to $1.1 \mathrm{TeV}$ for a nearly massless neutralino.

The previous ATLAS run-2 search in Ref. [58] made use of two event categories: events where one of the two tau leptons decays leptonically and the other hadronically were considered in addition to events where both tau leptons decay hadronically. While the branching fractions are almost the same for both categories, the leptonic decay of the tau lepton yields one neutrino more, which washes out the kinematic distributions and on average leads to a lower energy fraction carried by the lepton compared to the visible decay products from a hadronic tau-lepton decay. Taken together, the two effects significantly reduce the discriminative power of the selection requirements. As the sensitivity of the search is thus dominated by the category where both tau leptons decay hadronically, this paper considers only events with hadronically decaying tau leptons. These events are separated in two event categories (channels). One category selects events with at least two hadronically decaying tau leptons but no lighter leptons, at least one $b$-jet and large missing transverse momentum $E_{\mathrm{T}}^{\text {miss }}$ (di-tau channel). The other category selects events with exactly one hadronically decaying tau lepton, no electrons or muons, at least two $b$-jets and large $E_{\mathrm{T}}^{\text {miss }}$ (single-tau channel). The latter channel extends the sensitivity by covering the signal parameter space where the tau slepton is relatively light and one of the soft tau leptons easily escapes detection. Importantly, it also provides good sensitivity to events with pair-produced leptoquarks that decay into third-generation particles, which correspond to the second benchmark model.

The second benchmark model used in the design of the analysis considers pair production of scalar leptoquarks. It assumes that these only couple to third-generation quarklepton pairs, following the minimal Buchmüller-RücklWyler (BRW) model [60]. In addition to the coupling to the
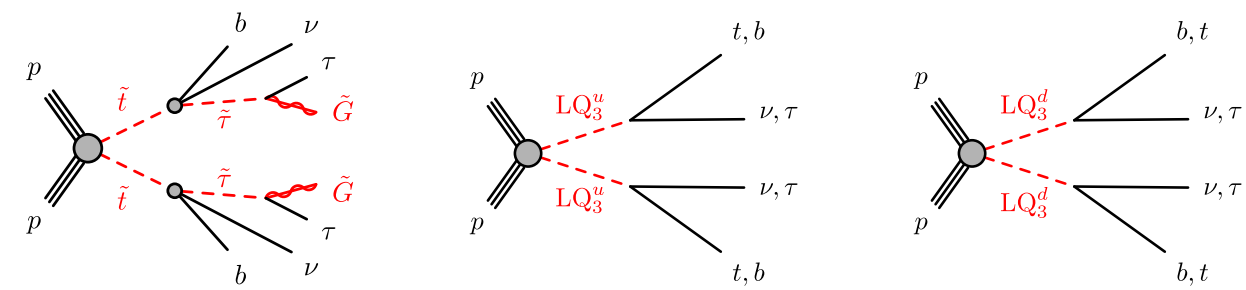

FIG. 1. Diagrams illustrating the production and decay of particles considered in the simplified models for the supersymmetric "stopstau" scenario (left) and for scalar leptoquarks of charge $+\frac{2}{3} e$ (middle) and $-\frac{1}{3} e$ (right). 
third fermion generation that is probed in this analysis, leptoquarks would need to have cross-generational couplings in order to explain the anomalies observed in $B$ meson decays. The search is carried out for both up-type scalar leptoquarks with fractional charge $Q\left(\mathrm{LQ}_{3}^{\mathrm{u}}\right)=+\frac{2}{3} e$ and decays $\mathrm{LQ}_{3}^{\mathrm{u}} \rightarrow t \nu_{\tau} / b \tau$, and down-type scalar leptoquarks with $Q\left(\mathrm{LQ}_{3}^{\mathrm{d}}\right)=-\frac{1}{3} e$ and decays $\mathrm{LQ}_{3}^{\mathrm{d}} \rightarrow b \nu_{\tau} / t \tau$. The production and decay of the leptoquarks are illustrated in Fig. 1. The model parameters are the leptoquark mass $m\left(\mathrm{LQ}_{3}^{\mathrm{u} / \mathrm{d}}\right)$ and the branching fraction $B\left(\mathrm{LQ}_{3}^{\mathrm{u} / \mathrm{d}} \rightarrow\right.$ $q \ell)$ into a quark and a charged lepton. For a branching fraction $B\left(\mathrm{LQ}_{3}^{\mathrm{u} / \mathrm{d}} \rightarrow q \ell\right) \sim 0.5$, most of the decays of the pair of third-generation leptoquarks yield a final state with one tau lepton, two $b$-jets and large $E_{\mathrm{T}}^{\text {miss }}$ from the tau neutrino. This signature matches that of the single-tau channel, which presents unique coverage of leptoquark masses at $B\left(\mathrm{LQ}_{3}^{\mathrm{u} / \mathrm{d}} \rightarrow q \ell\right) \sim 0.5$, but also has good sensitivity to a wide range of smaller or larger branching fractions.

The scalar-LQ model is the same as was used in a previous ATLAS paper [61] detailing a search for thirdgeneration leptoquarks based on $36.1 \mathrm{fb}^{-1}$ of data taken at $\sqrt{s}=13 \mathrm{TeV}$. This earlier paper comprises a dedicated reoptimization of the ATLAS search for pair-produced Higgs bosons and four reinterpretations of ATLAS SUSY searches, one of which is the previous iteration of the stopstau search [58]. Leptoquark masses below at least $0.8 \mathrm{TeV}$ are excluded at intermediate values of the branching fraction $B\left(\mathrm{LQ}_{3}^{\mathrm{u} / \mathrm{d}} \rightarrow q \ell\right)$, with the lower limit increasing at both small and large $B\left(\mathrm{LQ}_{3}^{\mathrm{u} / \mathrm{d}} \rightarrow q \ell\right)$, e.g., to 0.96 (1.02) $\mathrm{TeV}$ at $B\left(\mathrm{LQ}_{3}^{\mathrm{u} / \mathrm{d}} \rightarrow q \ell\right)=0$ (1) for down-type (up-type) leptoquarks. Two recent ATLAS searches for top or bottom squark pair production have been reinterpreted in the same up-type or down-type leptoquark model, respectively [50,62]. Another recent dedicated ATLAS search for pair-produced leptoquarks combines several event categories which all require at least one hadronically decaying tau lepton plus at least one electron or muon [63] and are complementary to the final states considered in this paper. It targets the down-type leptoquark model and excludes leptoquark masses up to $1.43 \mathrm{TeV}$ assuming $B\left(\mathrm{LQ}_{3}^{\mathrm{u} / \mathrm{d}} \rightarrow q \ell\right)=1$ and up to $1.22 \mathrm{TeV}$ assuming $B\left(\mathrm{LQ}_{3}^{\mathrm{u} / \mathrm{d}} \rightarrow q \ell\right)=0.5$. The CMS Collaboration has published a search of the full run-2 dataset for single or pair production of scalar or vector leptoquarks coupling to thirdgeneration fermions, considering final-state signatures consisting of a top quark, a tau lepton, a neutrino, and either no or at least one additional $b$-tagged jet. This search excludes scalar leptoquarks with masses up to about 1.0 TeV [64]. CMS has also reported several searches for third-generation leptoquarks based on $35.9 \mathrm{fb}^{-1}$ of run2 data [65-69], which typically set lower limits on the mass of scalar leptoquarks in the range of 0.9 to $1.1 \mathrm{TeV}$.
An additional interpretation of the search results is provided for pair production of vector leptoquarks $L_{3}^{v}$. Again, it is assumed that the vector leptoquarks can only decay into third-generation SM particles. The electric charge of the vector leptoquarks and their decay modes are the same as those of the up-type scalar leptoquarks in the middle diagram of Fig. 1. The signal selection criteria were not explicitly optimized for this model, but the kinematic distributions of the decay products are similar for scalar and vector leptoquarks, except when the branching fraction of the leptoquarks into a quark and a charged lepton is small, where tau leptons and $b$-jets predominantly arise from the leptoquarks decaying into top quarks and neutrinos rather than directly from the leptoquark decays. The signal selection developed for scalar leptoquarks can thus be expected to also perform very well for the case of vector leptoquarks, although the relevant energy scales are slightly higher in this case due to the larger production cross sections at the same mass. As in the signal model with scalar leptoquarks, the parameters for the vector-leptoquark model are the leptoquark mass $m\left(\mathrm{LQ}_{3}^{\mathrm{v}}\right)$ and the branching fraction $B\left(\mathrm{LQ}_{3}^{\mathrm{V}} \rightarrow b \tau\right)$ into a quark and a charged lepton. This is the first time this model is used in a search for leptoquarks by the ATLAS Collaboration. Models with vector leptoquarks have been considered in several analyses by the CMS Collaboration, including the one in Ref. [64], which excludes vector leptoquarks decaying into $t \bar{\nu}_{\tau} / b \tau^{+}$with masses up to $1.65 \mathrm{TeV}$ for pair production in the most favorable coupling scenario.

\section{ATLAS DETECTOR}

The ATLAS experiment [70-72] at the LHC is a multipurpose particle detector with a forward-backward symmetric cylindrical geometry and a near $4 \pi$ coverage in solid angle. ${ }^{1}$ It consists of an inner tracking detector surrounded by a thin superconducting solenoid providing a $2 \mathrm{~T}$ axial magnetic field, electromagnetic and hadronic calorimeters, and a muon spectrometer. The inner tracking detector covers the pseudorapidity range $|\eta|<2.5$. It consists of silicon pixel, silicon microstrip, and transition radiation tracking detectors. Lead/liquid-argon (LAr) sampling calorimeters provide electromagnetic (EM) energy measurements with high granularity. A steel/scintillator-tile hadronic calorimeter covers the central pseudorapidity range $(|\eta|<1.7)$. The end cap and forward regions are instrumented with LAr calorimeters for EM and hadronic energy measurements up to

\footnotetext{
${ }^{1}$ ATLAS uses a right-handed coordinate system with its origin at the nominal interaction point (IP) in the center of the detector and the $z$-axis along the beam pipe. The $x$-axis points from the IP to the center of the LHC ring, and the $y$-axis points upwards. Cylindrical coordinates $(r, \phi)$ are used in the transverse plane, $\phi$ being the azimuthal angle around the $z$-axis. The pseudorapidity is defined in terms of the polar angle $\theta$ as $\eta \equiv-\ln \tan (\theta / 2)$ and is an approximation of the rapidity $y \equiv 0.5 \ln \left[\left(E+p_{z}\right) /\left(E-p_{z}\right)\right]$ in the high-energy limit.
} 
$|\eta|=4$.9. The muon spectrometer surrounds the calorimeters and is based on three large superconducting air-core toroidal magnets with eight coils each. The muon spectrometer includes a system of precision tracking chambers and fast detectors for triggering. A two-level trigger system is used to select events [73]. The first-level trigger is implemented in hardware and uses a subset of the detector information to accept events at a rate below $100 \mathrm{kHz}$. This is followed by a software-based high-level trigger that reduces the accepted event rate to $1 \mathrm{kHz}$ on average depending on the data-taking conditions. An extensive software suite [74] is used for real and simulated data reconstruction and analysis, for operation and in the trigger and data acquisition systems of the experiment.

\section{DATA AND SIMULATED EVENT SAMPLES}

The dataset used in this analysis was collected with the ATLAS detector in proton-proton collisions provided by the LHC during its second run from 2015 to 2018. The data was taken at a center-of-mass energy of $\sqrt{s}=13 \mathrm{TeV}$ with a minimum separation of $25 \mathrm{~ns}$ between consecutive crossings of proton bunches from the two beams. Events are selected with triggers on missing transverse momentum [75], and data-quality requirements are applied to ensure that all elements of the detectors were operational during data-taking [76]. The total integrated luminosity amounts to $139 \mathrm{fb}^{-1}$ with an uncertainty of $1.7 \%$ [77], obtained using the LUCID-2 detector [78] for the primary luminosity measurements.

Monte Carlo (MC) simulation was used to generate samples of collision events, which model the expected kinematics of the investigated signal and SM background processes. Table I gives a detailed summary of the generation of the different MC samples used in the analysis. It lists the generators, the order of the cross section computation, the parton distribution function (PDF) sets, and the sets of tuned parameters (tunes) for the parton shower (PS). For background processes, the detector response was simulated [79] using the full modeling of the ATLAS detector in GEANT4 [80], while for the signal samples a faster variant of the simulation was used that relies on a parametrized response of the calorimeters [81]. Except for samples produced with SHERPA [82], which uses a dedicated parton-shower modeling and parameter tune developed by the SHERPA authors, the parton shower and hadronization simulation for all samples used the A14 tune [83], and the EVTGEN program [84] was used to model the decays of $b$ - and $c$-hadrons in signal samples and background events. The effect of multiple concurrent interactions in the same and neighboring bunch crossings (pileup) was modeled by overlaying the hard-scattering events with simulated inelastic $p p$ events generated with PYTHIA8.186 [85] using the NNPDF2.3LO set of PDFs [86] and the A3 tune [87]. All simulated events are processed with the same trigger, reconstruction and identification

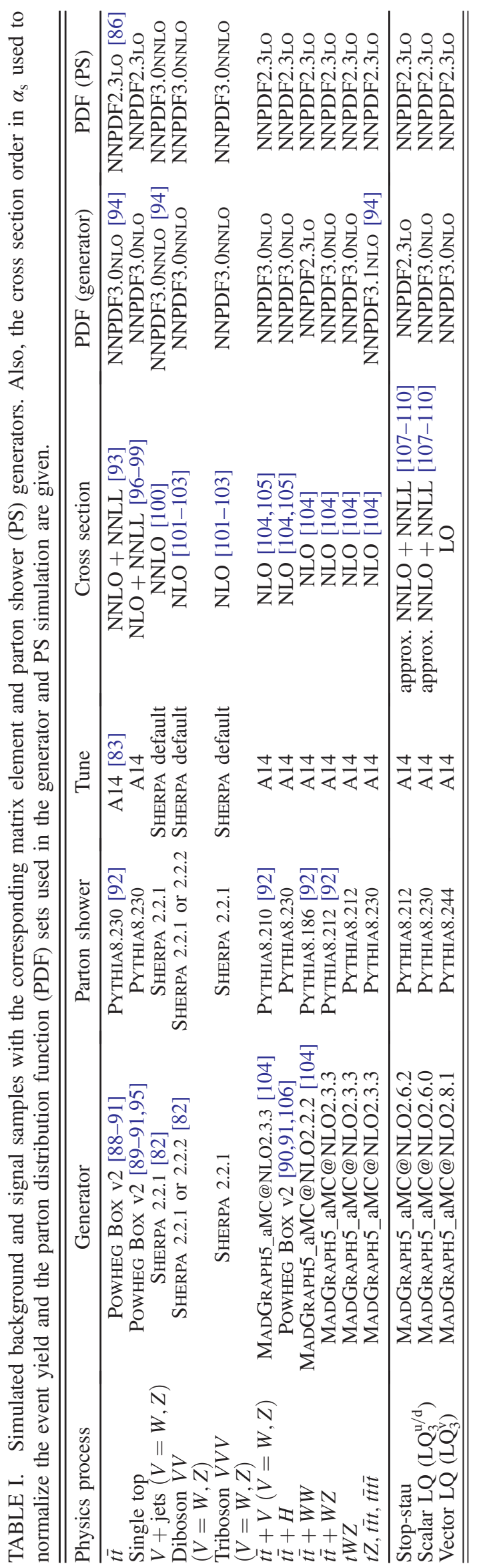


algorithms as the data, and are weighted to match the observed distribution of the pileup in data. Dedicated correction factors are applied to simulation to account for differences in efficiencies and energy calibrations between recorded data and simulations. In this analysis, data-driven methods are applied that improve the modeling of the dominant SM background processes by normalizing their contributions to data. These are described in Sec. VI.

The production of top-quark pairs, with or without an associated Higgs boson, and of single top quarks in the $s$ - or $t$ channel or associated with $W$ bosons was simulated with POWHEG Box [88-91], while associated production of topquark pairs and a vector boson $V=W$ or $Z$, as well as topquark production in other processes (later called "other top") giving smaller contributions $(t \bar{t}+W W, t \bar{t}+W Z, t W Z, t Z$, $t \bar{t} t$ and $t \bar{t} t \bar{t}$ ), was simulated with MADGRAPH5_aMC@NLO [104]. The events were interfaced to PyTHIA [92] to model the parton shower, hadronization, and underlying event, using the NNPDF2.3LO set of PDFs [86]. The production of single vector boson ( $V+$ jets), diboson $(V V)$ and triboson $(V V V)$ events was simulated with SHERPA using the NNPDF3.0NLO PDF set [94].

Stop-stau signal samples were produced for various values of $m(\tilde{t})$ and $m(\tilde{\tau})$. The pair production of top squarks was simulated at leading order with up to two additional partons in MADGrAPH5_aMC@NLO. For the decays of the SUSY particles, the top squark and the tau slepton, MADSPIN [111] was used to preserve spin correlation and finite-width effects. Both decays are assumed to be prompt; i.e., the SUSY particles have a negligible lifetime. The subsequent decays as well as the hadronization were simulated in PYTHIA. Cross sections are calculated including approximate next-to-next-to-leading-order (NNLO) supersymmetric quantum chromodynamics (QCD) corrections, with resummation of next-to-next-toleading logarithmic (NNLL) soft gluon terms [107-110]. The matching of matrix element and parton shower was done with the CKKW-L prescription [112,113], with the matching scale set to one quarter of the top-squark mass.

Simulated events with pair production of up- or downtype scalar third-generation leptoquarks $\mathrm{LQ}_{3}^{\mathrm{u} / \mathrm{d}}$ were generated at next-to-leading order (NLO) in QCD with MADGRAPH5_aMC@NLO, using the LQ model of Ref. [114] that adds parton showers to previous fixed-order NLO QCD calculations [115,116], and the NNPDF3.0NLO parton distribution function set with $\alpha_{\mathrm{s}}\left(m_{Z}\right)=0.118$. MADSPIN was used for the prompt decays of the leptoquarks into spin-entangled quark-lepton pairs of the third generation. Parton showering and hadronization were simulated in PYTHIA with the NNPDF2.3LO PDF set with $\alpha_{\mathrm{s}}\left(m_{Z}\right)=0.130$. The couplings in the Yukawa-type interaction of the leptoquarks with the quark-lepton pair are determined by two parameters: a common coupling strength $\lambda$ and an additional parameter $\beta$, with the coupling to a quark and a charged lepton given by $\sqrt{\beta} \lambda$, and the coupling to a quark and a neutrino by $\sqrt{1-\beta} \lambda$. The branching fraction $B\left(\mathrm{LQ}_{3}^{\mathrm{u} / \mathrm{d}} \rightarrow q \ell\right)$ into a quark and a charged lepton is, except for kinematic effects arising from the mass differences of the decay products, equal to $\beta$. The leptoquark signal samples were generated for various leptoquark masses $m\left(\mathrm{LQ}_{3}^{\mathrm{u} / \mathrm{d}}\right)$ and with a fixed parameter value of $\beta=0.5$, so that both decays of the leptoquarks, either into a quark and a neutrino or into a quark and a charged lepton, were possible. These events can be reweighted to arbitrary branching fractions $B\left(\mathrm{LQ}_{3}^{\mathrm{u} / \mathrm{d}} \rightarrow q \ell\right)$ to derive the interpretation of the analysis results in the plane of $m\left(\mathrm{LQ}_{3}^{\mathrm{u} / \mathrm{d}}\right)$ vs $B\left(\mathrm{LQ}_{3}^{\mathrm{u} / \mathrm{d}} \rightarrow q \ell\right)$. The coupling parameter $\lambda$ was set to 0.3 , close to the numeric value of the electromagnetic coupling $e=\sqrt{4 \pi \alpha}$, resulting in a $\mathrm{LQ}_{3}^{\mathrm{u} / \mathrm{d}}$ width equal to about $0.2 \%$ of its mass $[60,117]$. The cross sections for direct top-squark pair production are used for $\mathrm{LQ}_{3}^{\mathrm{u} / \mathrm{d}}$ pair production, as both involve massive, scalar, color-charged particles and the production modes are the same. These cross sections do not include the lepton $t$-channel contributions possible for LQ pair production, which are also neglected in Ref. [114] and may lead to corrections at the percent level [118].

Simulated events with pair production of thirdgeneration vector leptoquarks $\mathrm{LQ}_{3}^{\mathrm{v}}$ were generated at leading order in QCD with MADGRAPH5_aMC@NLO, using the LQ model of Ref. [119] and the NNPDF3.0NLO parton distribution function set with $\alpha_{\mathrm{s}}\left(m_{Z}\right)=0.118$. MADSPIN was used for the prompt decays of the leptoquarks, and parton showering and hadronization were simulated in PYTHIA with the NNPDF2.3LO PDF set with $\alpha_{\mathrm{S}}\left(m_{Z}\right)=0.130$. The $\mathrm{LQ}_{3}^{\mathrm{v}}$ in this model corresponds to the $U_{1}$ state in the BRW classification [60] and carries an electric charge of $Q\left(\mathrm{LQ}_{3}^{\mathrm{v}}\right)=+\frac{2}{3} e$. The model includes two additional vector states that are needed to obtain a realistic extension of the SM, a color singlet $Z^{\prime}$ and a color octet $G^{\prime}$. However, the $Z^{\prime}$ and $G^{\prime}$ do not appear in the Feynman diagrams considered for pair production of vector leptoquarks, as their interactions with the vector leptoquarks are not included in the model. All $\beta$ parameters are set to zero except for $\beta_{\mathrm{L}}^{33}$, such that only decays to left-chiral fermion fields are allowed, for which the coupling strength is set to $g_{U}=3.0$. The large value of $g_{U}$ is motivated by a suppression of the production cross section for additional mediators in a ultraviolet completion of the model, which might otherwise be in tension with LHC limits if these mediators are as light as needed to be consistent with the range of LQ masses considered here. As no higher-order computations of the cross sections are available for this vector-leptoquark model, the leading-order cross sections computed by the event generator are used. Two different scenarios are considered: the minimal-coupling scenario with $\kappa_{U}=\tilde{\kappa}_{U}=1$, where the LQ couples to the SM gauge bosons purely through the covariant derivative, and the Yang-Mills scenario with $\kappa_{U}=\tilde{\kappa}_{U}=0$, where the LQ is 
a massive gauge boson and has additional couplings to the SM gauge bosons [120]. The two scenarios differ mainly in the pair-production cross section, which is roughly 5 times as large in the Yang-Mills scenario at $m\left(\mathrm{LQ}_{3}^{\mathrm{v}}\right)=1.5 \mathrm{TeV}$ as in the minimal-coupling scenario, which in turn is roughly 4 times as large as the pair-production cross section for the scalar $\mathrm{LQ}_{3}^{\mathrm{u} / \mathrm{d}}$ at the same mass.

\section{EVENT RECONSTRUCTION}

All events are required to have at least one reconstructed interaction vertex with a minimum of two associated tracks with $p_{\mathrm{T}}>500 \mathrm{MeV}$. In events with multiple vertices, the one with the highest sum of squared transverse momenta of associated tracks is chosen as the primary vertex [121]. Events that contain jets that do not satisfy the set of quality criteria described in Ref. [122] are rejected in order to reduce noncollision backgrounds and backgrounds induced by calorimeter noise.

Jets are reconstructed from particle-flow objects [123] calibrated at the EM scale using the anti- $k_{t}$ algorithm with a radius parameter of $R=0.4[124,125]$. Since both signal models predict the production of particles with large masses, only jets in the central region within $|\eta|<2.8$ are used. The jets are calibrated following the procedure described in Ref. [126] and are required to have $p_{\mathrm{T}}>20 \mathrm{GeV}$. To suppress jets from pileup interactions, jet candidates with $p_{\mathrm{T}}<60 \mathrm{GeV}$ and $|\eta|<2.4$ are required to pass the tight working point of the jet vertex tagger [127]. Selected jets that are likely to originate from the hadronization of a bottom quark are flagged as $b$-jets if they lie within $|\eta|<2.5$ and are tagged by the DLIr algorithm, a multivariate discriminant based on various inputs such as track impact parameters and displaced secondary vertices $[128,129]$. The $b$-tagging algorithm uses a working point with an efficiency of $77 \%$, with an approximate misidentification probability of $20 \%$ for jets arising from charm quarks, $6.7 \%$ for hadronically decaying $\tau$-leptons, and $0.9 \%$ for light-flavor jets in simulated $t \bar{t}$ events.

Tau leptons which decay leptonically are not identified as such, but are instead reconstructed as a candidate for a prompt electron or muon. Therefore, in the context of reconstructed analysis objects, "tau lepton" will always refer to a hadronic tau lepton, i.e., a tau lepton that decays hadronically. The visible component of hadronically decaying tau leptons is reconstructed from anti- $k_{t}$ jets $(R=0.4)$ built from locally calibrated topological clusters [130], with a distance parameter $R=0.4$ and requiring $p_{\mathrm{T}}>10 \mathrm{GeV}$ and $|\eta|<2.5[131,132]$. The energy calibration applies a pileup subtraction and a correction to the detector response. Information from the tracking system improves the energy resolution at low $p_{\mathrm{T}}[132,133]$. Taulepton candidates are required to have $p_{\mathrm{T}}>20 \mathrm{GeV}$ and lie outside the transition region $1.37<|\eta|<1.52$ between the barrel and end cap calorimeters. Furthermore, they must have either one or three charged tracks ("prongs") with a charge sum of \pm 1 in units of the elementary charge. A recurrent neural network algorithm [134] distinguishes hadronically decaying tau leptons from quark- and gluoninitiated jets by using a combination of high-level discriminating variables as well as tracking and calorimeter measurements. Its medium working point is used to identify hadronic tau leptons, with efficiencies of $75 \%$ and $60 \%$ in simulated Drell-Yan events, and background-rejection factors of 35 and 240 in simulated dijet events, for one-prong and three-prong decays, respectively. Electrons misidentified as hadronic tau-lepton candidates are rejected using a dedicated boosted decision tree algorithm. Reconstructed tau leptons in simulated events are called "real" tau leptons if they can be geometrically matched to a tau lepton in the MC "truth" record; otherwise they are referred to as "fake" tau leptons.

As described in Sec. V, events with prompt electrons or muons are rejected in the analysis selections, so these only enter in the computation of missing transverse momentum and in the overlap-removal procedure, and are not considered otherwise. Electron candidates are reconstructed from energy deposits in the electromagnetic calorimeter that are matched to tracks in the inner detector (ID) $[135,136]$. They are required to have $p_{\mathrm{T}}>10 \mathrm{GeV}$ and $|\eta|<2.47$ and pass the LooseAndBLayer identification requirement. Muon candidates are reconstructed by combining information from the ID and the muon spectrometer [137]. They are required to have $p_{\mathrm{T}}>10 \mathrm{GeV}$ and $|\eta|<2.7$ and satisfy the medium identification criteria. The absolute value of the longitudinal impact parameter $z_{0}$ of each prompt electron or muon candidate is required to be less than $0.5 \mathrm{~mm}$.

An overlap-removal procedure is applied to all selected objects to resolve ambiguities in the reconstruction in several consecutive steps. First, if two electrons share the same track, the electron with lower transverse momentum is discarded. Next, tau leptons overlapping with an electron or a muon within $\Delta R_{y}<0.2$ are removed, where the angular distance is measured in units of $\Delta R_{y} \equiv$ $\sqrt{(\Delta y)^{2}+(\Delta \phi)^{2}}$ with the rapidity $y$ instead of the pseudorapidity $\eta$ to account for cases where particle masses cannot be neglected. If an electron shares an ID track with a muon, the electron is discarded unless the muon is tagged as a minimum-ionizing particle in the calorimeter, in which case the muon is discarded. Jets within $\Delta R_{y}=0.2$ of an electron are removed. In order to suppress electrons from semileptonic heavy-flavor decays, electrons within $\Delta R_{y}=0.4$ of a jet are removed. Any jet with fewer than three associated tracks is discarded if a muon is within $\Delta R_{y}=0.2$ of the jet or if a muon can be matched to a track associated with the jet. For the same reason as for electrons, muons within $\Delta R_{y}=0.4$ of a jet are removed. Lastly, jets within $\Delta R_{y}=0.4$ of a tau lepton are removed.

The missing transverse momentum $\mathbf{E}_{\mathrm{T}}^{\text {miss }}$ is defined as the negative vector sum of the transverse momenta of all calibrated objects mentioned above, photons [136], and an 
additional soft term including all tracks associated with the primary vertex but not matched to any reconstructed object $[138,139]$. The magnitude of $\mathbf{E}_{\mathrm{T}}^{\text {miss }}$ is denoted by $E_{\mathrm{T}}^{\text {miss }}$.

\section{EVENT SELECTION}

The analysis covers two different channels: the singletau channel and the di-tau channel. In both channels, object multiplicities and kinematic variables are used to define several different event selections (analysis regions). All of these event selections start from a common preselection described below. The preselections in the single-tau and ditau channels are identical except for the number of tau leptons and $b$-tagged jets. The sets of events selected in the two channels are thus mutually exclusive and can therefore be statistically combined, as is done in the interpretation of the results.

\section{A. Preselection}

The preselection requirements for the two channels are summarized in Table II. Events are selected using an $E_{\mathrm{T}}^{\text {miss }}$ trigger [75]. In combination with the requirement of $E_{\mathrm{T}}^{\text {miss }}>250 \mathrm{GeV}$, this trigger is fully efficient in the phase space that the analysis targets. As no light leptons are expected from the benchmark signal models when only hadronically decaying tau leptons are considered, events with light leptons are rejected. Events are required to have at least two jets, at least one (two) of which must be $b$-tagged in the di-tau (single-tau) channel. Additionally, events in the di-tau channel are required to have at least two reconstructed tau leptons, whereas exactly one tau lepton is required in the single-tau channel. The tight $E_{\mathrm{T}}^{\text {miss }}$ and $b$-tagging requirements efficiently suppress multijet events such that their contribution to the analysis regions is negligible. This was verified with dedicated data-driven estimates for both channels.

\section{B. Signal regions}

Dedicated signal-enriched regions are defined for each channel, having been optimized individually by maximizing the estimated discovery significance [140] for benchmark signal models close to the previous exclusion contours. The selection requirements for the signal regions are explained in the following, and a summary is included

TABLE II. Preselection of the di-tau and single-tau channels.

\begin{tabular}{|c|c|}
\hline Di-tau preselection & Single-tau preselection \\
\hline \multicolumn{2}{|c|}{$E_{\mathrm{T}}^{\mathrm{miss}}$-trigger fired and $E_{\mathrm{T}}^{\mathrm{miss}}>250 \mathrm{GeV}$} \\
\hline \multicolumn{2}{|c|}{ No light leptons $(e / \mu)$} \\
\hline \multicolumn{2}{|c|}{ At least two jets } \\
\hline $\begin{array}{l}\text { At least two hadronic } \\
\text { tau leptons }\end{array}$ & Exactly one hadronic tau lepton \\
\hline At least one $b$-tagged jet & At least two $b$-tagged jets \\
\hline
\end{tabular}

in the overview of the analysis regions in Table III for the di-tau channel and Table IV for the single-tau channel. The signal region (SR) in the di-tau channel targets stop-stau signal models with a low to modest mass difference between the top squark and the tau slepton. This SR is not used for the leptoquark models, as the final states for that model at $\beta=0.5$ have only one tau lepton on average. The case of $\beta=1.0$, which would yield two tau leptons, is not within the scope of this paper, and the requirements on $E_{\mathrm{T}}^{\mathrm{miss}}$ and that no leptons be present in the final state strongly reduce the sensitivity to this scenario. The singletau channel employs two signal regions: a one-bin SR for the model-independent fit, and a multibin SR for the modeldependent fit, as is discussed in Sec. VIII. Each of the two signal regions in this channel is optimized simultaneously for the scalar-leptoquark signal models and the stop-stau signal models that have a large mass difference between the top squark and the tau slepton.

\section{Di-tau channel}

The most discriminating variable in the di-tau channel is the "stransverse" mass variable [141,142], which by itself already provides good separation between the signal and the background. The stransverse mass $m_{\mathrm{T} 2}$ is a generalization of the transverse mass $m_{\mathrm{T}}$, which is computed as $m_{\mathrm{T}}{ }^{2}\left(\mathbf{p}_{\mathrm{T}}, \mathbf{E}_{\mathrm{T}}^{\text {miss }}\right)=2 p_{\mathrm{T}} E_{\mathrm{T}}^{\text {miss }}\left(1-\cos \Delta \phi\left(\mathbf{p}_{\mathrm{T}}, \mathbf{E}_{\mathrm{T}}^{\text {miss }}\right)\right) \quad$ from the transverse momentum of some given particle and the missing transverse momentum. It generalizes the transverse mass for symmetric event topologies where two identical particles each decay into a visible and an invisible product. In this case the individual transverse momenta of the invisible particles can no longer be directly approximated by the measured missing transverse momentum, as the information about their individual contributions to the missing transverse momentum is lost. Using subscripts to refer to the physics objects reconstructed in a collision event in order of decreasing transverse momentum, for the two leading tau leptons, i.e., the two tau leptons with the largest $\left(\tau_{1}\right)$ and second-largest $\left(\tau_{2}\right)$ transverse momentum, $m_{\mathrm{T} 2}\left(\tau_{1}, \tau_{2}\right)$ is computed as

$$
m_{\mathrm{T} 2}\left(\tau_{1}, \tau_{2}\right)=\min _{\mathbf{q}_{\mathrm{T}}^{a}+\mathbf{q}_{\mathrm{T}}^{b}=\mathbf{E}_{\mathrm{T}}^{\text {miss }}}\left(\max \left[m_{\mathrm{T}}\left(\mathbf{p}_{\mathrm{T}}^{\tau_{1}}, \mathbf{q}_{\mathrm{T}}^{a}\right), m_{\mathrm{T}}\left(\mathbf{p}_{\mathrm{T}}^{\tau_{2}}, \mathbf{q}_{\mathrm{T}}^{b}\right)\right]\right),
$$

where $a$ and $b$ refer to two invisible particles assumed to be produced with transverse momentum $\mathbf{q}_{\mathrm{T}}^{a, b}$. The minimum is taken over all possible assignments to $\mathbf{q}_{\mathrm{T}}^{a, b}$ that sum to the measured $\mathbf{E}_{\mathrm{T}}^{\text {miss }}$. The masses of the invisible particles are free parameters and are set to zero. For the dominant topquark-related backgrounds, the $m_{\mathrm{T} 2}\left(\tau_{1}, \tau_{2}\right)$ distribution features an end point near the $W$-boson mass. By placing a lower bound at $70 \mathrm{GeV}$ most of this background can be removed, while efficiently selecting stop-stau signal events, for which the $m_{\mathrm{T} 2}\left(\tau_{1}, \tau_{2}\right)$ distribution exhibits a tail towards 
TABLE III. Definitions of the $t \bar{t}$ control and validation regions and the signal region in the di-tau channel. Centered dots $(\cdots)$ signify that no requirement on the given variable is applied, while brackets indicate an allowed range for the variable. These requirements extend those of the di-tau preselection from Table II.

\begin{tabular}{|c|c|c|c|c|c|}
\hline Variable & $\mathrm{CR} t \bar{t}(2$ real $\tau)$ & $\mathrm{CR} t \bar{t}(1$ real $\tau)$ & $\mathrm{VR} t \bar{t}(2$ real $\tau)$ & $\operatorname{VR} t \bar{t}(1 \operatorname{real} \tau)$ & SR \\
\hline$E_{\mathrm{T}}^{\mathrm{miss}}$ & $\ldots$ & $\cdots$ & $\cdots$ & $\cdots$ & $>280 \mathrm{GeV}$ \\
\hline$O S\left(\tau_{1}, \tau_{2}\right)$ & 1 & $\ldots$ & 1 & $\cdots$ & 1 \\
\hline$m_{\mathrm{T} 2}\left(\tau_{1}, \tau_{2}\right)$ & $<35 \mathrm{GeV}$ & $<35 \mathrm{GeV}$ & {$[35,70] \mathrm{GeV}$} & {$[35,70] \mathrm{GeV}$} & $>70 \mathrm{GeV}$ \\
\hline$m_{\mathrm{vis}}\left(\tau_{1}, \tau_{2}\right)$ & $>50 \mathrm{GeV}$ & $>50 \mathrm{GeV}$ & $\ldots$ & $\ldots$ & $\ldots$ \\
\hline$m_{\mathrm{T}}\left(\tau_{1}\right)$ & $>50 \mathrm{GeV}$ & $<50 \mathrm{GeV}$ & $>70 \mathrm{GeV}$ & $<70 \mathrm{GeV}$ & $\cdots$ \\
\hline
\end{tabular}

much higher values. The sensitivity is further enhanced by raising the lower bound on $E_{\mathrm{T}}^{\text {miss }}$ to $280 \mathrm{GeV}$ and requiring the two leading tau leptons to carry electric charges with opposite signs, a criterion later denoted by $\operatorname{OS}\left(\tau_{1}, \tau_{2}\right)=1$.

\section{Single-tau channel}

Both signal regions in the single-tau channel have a lower bound on $E_{\mathrm{T}}^{\text {miss }}$ at $280 \mathrm{GeV}$ and on the sum of the transverse masses of the $b$-jets, $\sum m_{\mathrm{T}}\left(b_{1,2}\right)=m_{\mathrm{T}}\left(b_{1}\right)+m_{\mathrm{T}}\left(b_{2}\right)$, at $700 \mathrm{GeV}$. In this expression and the following, $m_{\mathrm{T}}(A)$ for a given particle $A$ should be read as $m_{\mathrm{T}}(A) \equiv m_{\mathrm{T}}\left(\mathbf{p}_{\mathrm{T}}^{A}, \mathbf{E}_{\mathrm{T}}^{\text {miss }}\right)$. The one-bin SR requires $m_{\mathrm{T}}(\tau)>300 \mathrm{GeV}$ and $s_{\mathrm{T}}>$ $800 \mathrm{GeV}$, where $s_{\mathrm{T}}$ is defined as the scalar sum of the transverse momenta of the tau lepton and the two leading jets, $s_{\mathrm{T}}=p_{\mathrm{T}}(\tau)+p_{\mathrm{T}}\left(\mathrm{jet}_{1}\right)+p_{\mathrm{T}}\left(\mathrm{jet}_{2}\right)$. While both the stop-stau and $\mathrm{LQ}_{3}^{\mathrm{u} / \mathrm{d}}$ signals show fairly similar behavior in most kinematic variables, their $p_{\mathrm{T}}(\tau)$ distributions differ. This is due to the large mass difference in the stop-stau target scenario, so that the tau leptons are softer than those produced in the $L Q_{3}^{\mathrm{u} / \mathrm{d}}$ decay. To account for the different shapes of the transverse momentum distributions of the tau leptons, the second SR is defined with three bins in $p_{\mathrm{T}}(\tau)$. The first two $p_{\mathrm{T}}(\tau)$ bins cover 50 to $100 \mathrm{GeV}$ and 100 to $200 \mathrm{GeV}$, and the last bin all values beyond $200 \mathrm{GeV}$. To reduce the statistical uncertainty in the three $p_{\mathrm{T}}(\tau)$ bins, two selection requirements are loosened relative to the one-bin SR: the minimum $m_{\mathrm{T}}(\tau)$ requirement is lowered to $150 \mathrm{GeV}$, and the minimum $s_{\mathrm{T}}$ requirement to $600 \mathrm{GeV}$. As the one-bin SR is a subset of the multibin SR, they cannot be combined in the statistical interpretation of the results discussed in Sec. VIII. A multibin SR based on $s_{\mathrm{T}}$ instead of $p_{\mathrm{T}}(\tau)$ was also tested but was found to have lower sensitivity.

\section{BACKGROUND ESTIMATION}

The background in the signal regions is dominated by $t \bar{t}$ and single-top production, which can yield events with a final state similar to the signal processes. Dedicated control regions are defined for these background processes. Topquark production can contribute to the background in different ways. Events with $t \bar{t}$ production, where both $W$ bosons arising from the top-quark decay into a hadronic tau lepton, have two real tau leptons. This process, denoted by $t \bar{t}$ (2 real $\tau$ ), contributes to the di-tau channel if both hadronic tau leptons are correctly identified. If instead only one of the $W$ bosons from the $t \bar{t}$ system gives a hadronic tau lepton which is correctly identified, and the second $W$ boson decays hadronically, the resulting jet from the second $W$-boson decay can be misidentified as a tau lepton, and such an event can then still satisfy the di-tau channel selection criteria. While the misidentification probability is of the order of a few percent, the larger branching fraction of hadronic $W$ decays and the less pronounced end point in the $m_{\mathrm{T} 2}\left(\tau_{1}, \tau_{2}\right)$ distribution for $t \bar{t}$ events with one real and one fake tau lepton still leads to a significant contribution in the di-tau channel. This type of event can also enter the single-tau channel selection, if the jet from the second $W$ boson is not misidentified as a tau lepton. Di-tau $t \bar{t}$ events in which only one of the two identified tau leptons is real, and single-tau $t \bar{t}$ events with one real tau lepton, are referred to as $t \bar{t}(1$ real $\tau)$ events. Lastly, fully hadronic $t \bar{t}$ decays, without any real tau leptons that pass the selections in either the single-tau or di-tau channel, are referred to as $t \bar{t}$-fake events. Due to their different kinematics, the simulated $t \bar{t}$

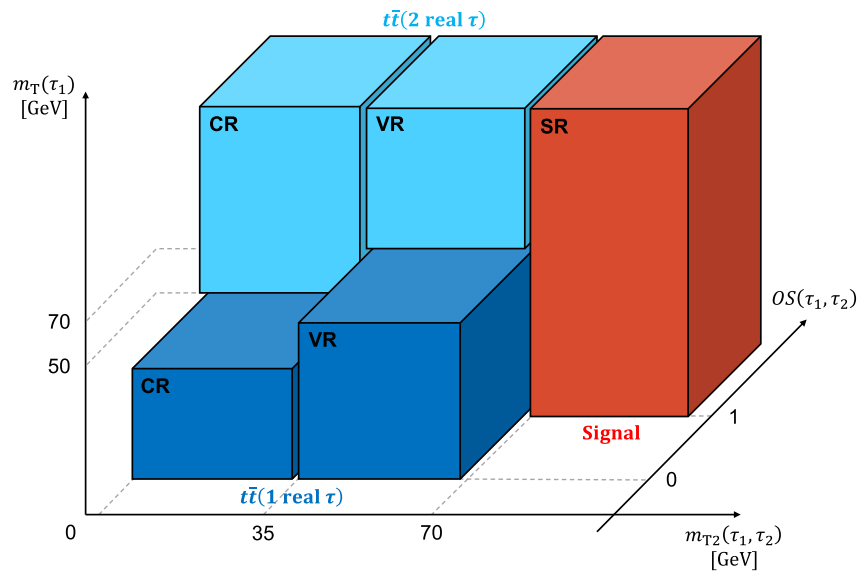

FIG. 2. Overview of the selections defining the control, validation and signal regions in the di-tau channel in the phase-space spanned by the variables $m_{\mathrm{T} 2}\left(\tau_{1}, \tau_{2}\right), m_{\mathrm{T}}\left(\tau_{1}\right)$, and $O S\left(\tau_{1}, \tau_{2}\right)$, where $O S\left(\tau_{1}, \tau_{2}\right)=1$ means that the reconstructed charges of the two leading tau leptons have opposite signs. In addition to these variables, $E_{\mathrm{T}}^{\mathrm{miss}}>280 \mathrm{GeV}$ is required for the signal region, and $m_{\text {vis }}\left(\tau_{1}, \tau_{2}\right)>50 \mathrm{GeV}$ for the control regions. The complete definitions are summarized in Table III. 
events are separated into these three event types, $t \bar{t}(2$ real $\tau), t \bar{t}(1$ real $\tau)$, and $t \bar{t}$-fake, and treated as separate background components in the following.

Subdominant contributions to the SM background arise from singly produced vector bosons $(W+$ jets and $Z+$ jets events) and production of vector bosons in association with top-quark pairs $(\bar{t}+V)$. In addition, multiboson production, $t \bar{t}$ production in association with a Higgs boson $(t \bar{t}+H)$ and other top-related processes yield small contributions. These subdominant processes are normalized according to the theory cross section predictions and the integrated luminosity measured in data.

The normalization factors for the MC predictions for $t \bar{t}$ and single-top production are extracted in a simultaneous binned maximum-likelihood fit to the observed data in the control regions (CRs). This fit, where no signal contributions are included, is referred to as the background-only fit. The CRs are designed to be enriched in a given background process and to be kinematically as similar to the SRs as possible, while maintaining sufficient purity and a high enough event yield with negligible contamination from signal. In addition to the data yields in the CRs, the expected yields and statistical and systematic uncertainties from MC simulation, described in Sec. VII, are input to the background-only fit. The yields obtained from the background-only fit can then be extrapolated to dedicated validation regions (VRs) to assess the accuracy of the background estimate. All CR, VR and SR selections are mutually exclusive so that they are statistically independent as required for the fit. The $\mathrm{CR}$ and VR selections are
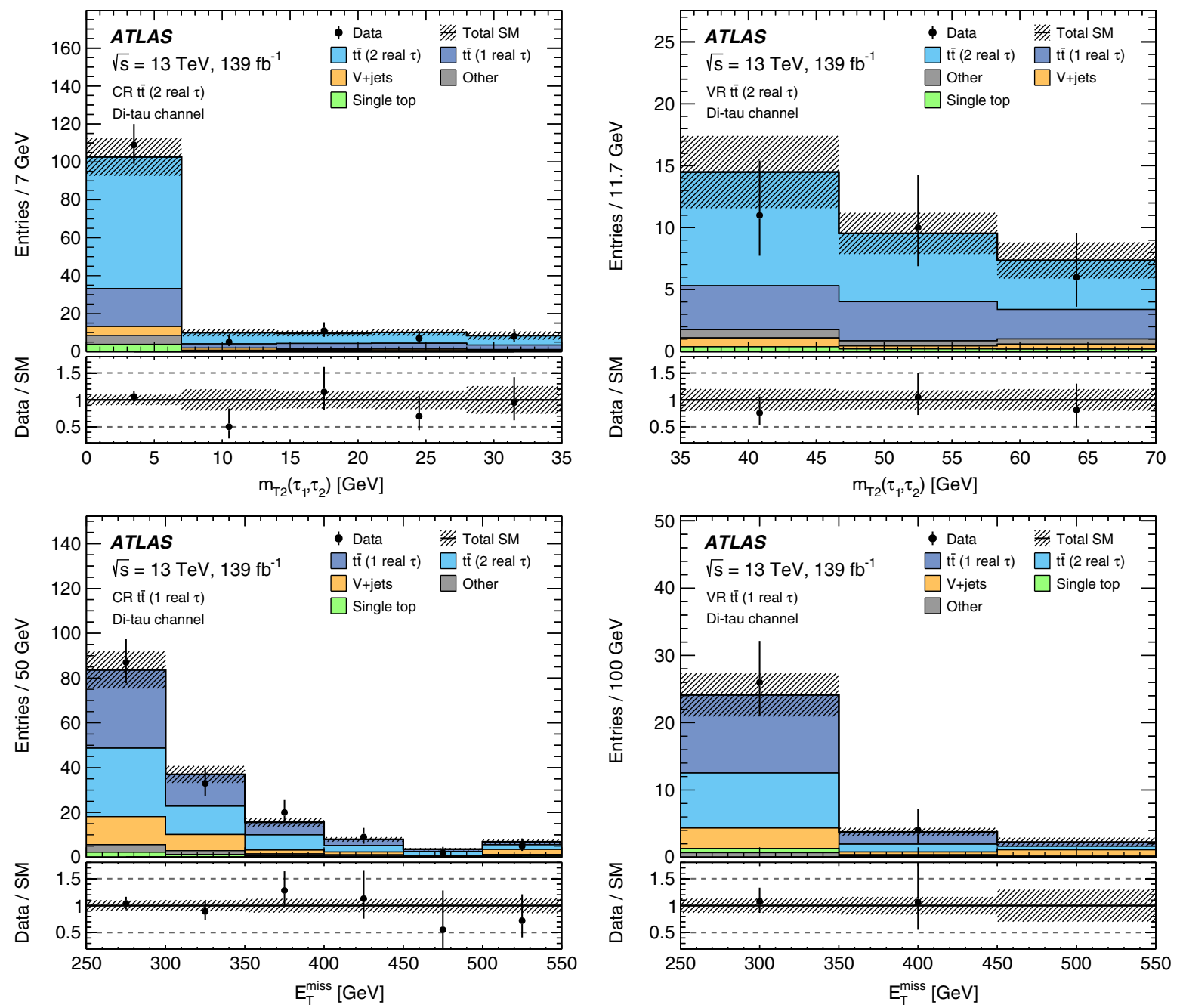

FIG. 3. Distributions of $m_{\mathrm{T} 2}\left(\tau_{1}, \tau_{2}\right)$ and $E_{\mathrm{T}}^{\text {miss }}$ in the di-tau channel. The left-hand plots show the control regions and the right-hand plots the validation regions, with $m_{\mathrm{T} 2}\left(\tau_{1}, \tau_{2}\right)$ in the $t \bar{t}(2$ real $\tau) \mathrm{CR}$ and VR in the top row and $E_{\mathrm{T}}^{\text {miss }}$ in the $t \bar{t}(1$ real $\tau) \mathrm{CR}$ and VR in the bottom row. The CRs and VRs have different requirements on the transverse mass $m_{\mathrm{T}}\left(\tau_{1}\right)$. The stacked histograms show the various SM background contributions. The hatched band indicates the total statistical and systematic uncertainty of the SM background. The $t \bar{t}$ ( 2 real $\tau$ ) and $t \bar{t}(1$ real $\tau$ ) contributions and the single-top background contributions are scaled with the normalization factors obtained from the background-only fit. Minor backgrounds are grouped together and denoted by "Other". This includes $t \bar{t}$-fake, $t \bar{t}+X$, multiboson, and other top. The rightmost bin includes the overflow. 


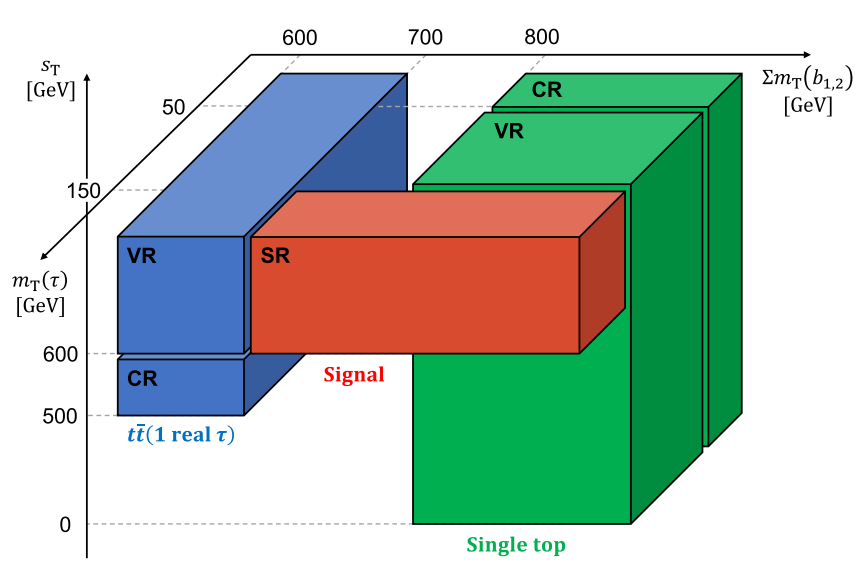

FIG. 4. Overview of the selections defining the control and validation regions and the multibin signal region in the single-tau channel in the phase-space spanned by the variables $\sum m_{\mathrm{T}}\left(b_{1,2}\right)$, $m_{\mathrm{T}}(\tau)$ and $s_{\mathrm{T}}$. In addition to these variables, $E_{\mathrm{T}}^{\text {miss }}>280 \mathrm{GeV}$ is required for the signal region, and $p_{\mathrm{T}}\left(\tau_{1}\right)>80 \mathrm{GeV}$ for the single-top control and validation regions. The complete definitions are summarized in Table IV.

introduced in Secs. VI A and VI B for the di-tau and singletau channel, respectively. In addition to the backgroundonly fit, model-dependent and model-independent fit setups [143] which include the signal regions are used for the interpretation of the results as further detailed in Sec. VIII.

\section{A. Di-tau channel}

Table III summarizes the selections that define the control, validation and signal regions in the di-tau channel. One control region and one validation region are defined in this channel for each of the $t \bar{t}(2$ real $\tau$ ) and $t \bar{t}(1$ real $\tau$ ) processes. Their location in the phase-space spanned by $m_{\mathrm{T} 2}\left(\tau_{1}, \tau_{2}\right), m_{\mathrm{T}}\left(\tau_{1}\right)$, and $O S\left(\tau_{1}, \tau_{2}\right)$ is illustrated in Fig. 2 . The CRs and VRs sit in the $m_{\mathrm{T} 2}\left(\tau_{1}, \tau_{2}\right)$ sideband below $70 \mathrm{GeV}$, above which the SR is located, and are separated at $35 \mathrm{GeV}$.

Top-quark pair-production events in which only one of the $W$ bosons decays leptonically, with one real tau lepton and one fake tau lepton, feature an end point in the $m_{\mathrm{T}}$ distribution of the real tau lepton near the $W$ mass. The reason is that the dominant source of $E_{\mathrm{T}}^{\text {miss }}$ is the tau neutrino from the $W$ decay. By contrast, for $t \bar{t}$ events with two real tau leptons, two tau neutrinos contribute to the $E_{\mathrm{T}}^{\text {miss }}$ and there is no distinct end point in $m_{\mathrm{T}}$. This difference in the shapes of the $m_{\mathrm{T}}$ distributions is exploited in the selection of $t \bar{t}(2 \mathrm{real} \tau)$ and $t \bar{t}(1 \mathrm{real} \tau)$ events. In the majority of $t \bar{t}$ ( 1 real $\tau$ ) events in the di-tau channel, the real tau lepton corresponds to the leading reconstructed tau lepton. A requirement on $m_{\mathrm{T}}\left(\tau_{1}\right)$ at 50 (70) $\mathrm{GeV}$ is thus used to separate the $t \bar{t}$ ( 2 real $\tau$ ) CR (VR) from the $t \bar{t}(1$ real $\tau)$ CR (VR). By requiring the leading and subleading tau lepton in the $t \bar{t}(2$ real $\tau$ ) CR and VR selections to carry electric charges of opposite sign, $O S\left(\tau_{1}, \tau_{2}\right)=1$, the purity is further increased. In addition, a lower bound on the invariant mass of the two tau leptons computed from the visible decay products, $m_{\mathrm{vis}}\left(\tau_{1}, \tau_{2}\right)$, at $50 \mathrm{GeV}$ is applied to reduce the contribution from $Z+$ jets events.

Distributions of the main discriminating variables $m_{\mathrm{T} 2}\left(\tau_{1}, \tau_{2}\right)$ and $E_{\mathrm{T}}^{\mathrm{miss}}$ in the control and validation regions of the di-tau channel are shown in Fig. 3. The predictions for the top-quark backgrounds are scaled with the normalization factors obtained from the background-only fit. Their values are given in Sec. VIII. From the plots it can be seen that the background model describes the data very well.

\section{B. Single-tau channel}

For the two dominant processes in the single-tau channel, $t \bar{t}$ production with one real tau lepton and single-top production, again two pairs of control and validation regions are defined. The definitions are illustrated in Fig. 4 and summarized in Table IV. In contrast to the di-tau CRs and VRs, the larger available number of events in the single-tau channel allows the lower bound on $E_{\mathrm{T}}^{\text {miss }}$ used in the CR and VR selections to be the same as for the SR. The $t \bar{t}$ ( 1 real $\tau$ ) control and validation regions in the single-tau channel are placed in the $\sum m_{\mathrm{T}}\left(b_{1,2}\right)$ sideband between 600 and $700 \mathrm{GeV}$. The control region is located in the $s_{\mathrm{T}}$ window from 500 to $600 \mathrm{GeV}$, and the validation

TABLE IV. Definitions of the $t \bar{t}$ ( 1 real $\tau)$ and single-top control and validation regions and the signal region in the single-tau channel. Centered dots $(\cdots)$ signify that no requirement on the given variable is applied, while brackets indicate an allowed range for the variable. In the last column, parentheses enclose the values and ranges used for the multibin SR. The binning in $p_{\mathrm{T}}(\tau)$ of the multibin SR, abbreviated with "binned," is [50, 100], [100, 200], and >200 GeV. These requirements extend those of the single-tau preselection from Table II.

\begin{tabular}{lccccc}
\hline \hline Variable & CR $t \bar{t}(1$ real $\tau)$ & CR single top & VR $t \bar{t}(1$ real $\tau)$ & VR single top & SR \\
\hline$E_{\mathrm{T}}^{\text {miss }}$ & $>280 \mathrm{GeV}$ & $>280 \mathrm{GeV}$ & $>280 \mathrm{GeV}$ & $>280 \mathrm{GeV}$ & $>280 \mathrm{GeV}$ \\
$s_{\mathrm{T}}$ & {$[500,600] \mathrm{GeV}$} & $\cdots$ & $>600 \mathrm{GeV}$ & $\cdots$ & $>800(600) \mathrm{GeV}$ \\
$\sum_{m_{\mathrm{T}}}\left(b_{1,2}\right)$ & {$[600,700] \mathrm{GeV}$} & $>800 \mathrm{GeV}$ & {$[600,700] \mathrm{GeV}$} & $>800 \mathrm{GeV}$ & $>700 \mathrm{GeV}$ \\
$m_{\mathrm{T}}(\tau)$ & $\cdots$ & $<50 \mathrm{GeV}$ & $\cdots$ & {$[50,150] \mathrm{GeV}$} & $>300(150) \mathrm{GeV}$ \\
\hline \hline
\end{tabular}


region covers the range above $600 \mathrm{GeV}$. The normalization of the $t \bar{t}(1$ real $\tau$ ) process is obtained from a simultaneous fit of both control regions for this process, one from each channel. The CR and VR for the single-top background sit in the $m_{\mathrm{T}}(\tau)$ window from 0 to $50 \mathrm{GeV}$ and 50 to $150 \mathrm{GeV}$, respectively. Additionally, lower bounds on $\sum m_{\mathrm{T}}\left(b_{1,2}\right)$ at $800 \mathrm{GeV}$ and on $p_{\mathrm{T}}(\tau)$ at $80 \mathrm{GeV}$ increase the purity of both the single-top CR and VR. Events from $t \bar{t}(1$ real $\tau$ ) are less likely to fulfill the $p_{\mathrm{T}}(\tau)$ requirement, which favors highenergy decay products. They also tend to have lower $\sum m_{\mathrm{T}}\left(b_{1,2}\right)$, as the transverse mass computed for the subleading $b$-jet has a quite distinct end point near the top-quark mass.

Figure 5 shows the distribution of $s_{\mathrm{T}}$ and $m_{\mathrm{T}}(\tau)$ in the $t \bar{t}$ ( 1 real $\tau$ ) CR and VR and in the single-top CR and VR of the single-tau channel. The predictions for the top-quark backgrounds are scaled with the normalization factors obtained from the background-only fit. These are consistent with one for the $t \bar{t}(2$ real $\tau$ ) and $t \bar{t}(1$ real $\tau$ ) backgrounds, but much smaller than one for the single-top background as discussed further in Section VIII. Therefore, the contribution of scaled single-top events to the single-top CR and VR in the figure is very low, whereas it is $43 \%$ before applying the normalization factors. From the plots it can be seen that the background model describes the data very well.

\section{SYSTEMATIC UNCERTAINTIES}

The expected yields for signal and background processes are subject to experimental and theoretical systematic uncertainties. These uncertainties are implemented as variations which are parametrized as functions of nuisance
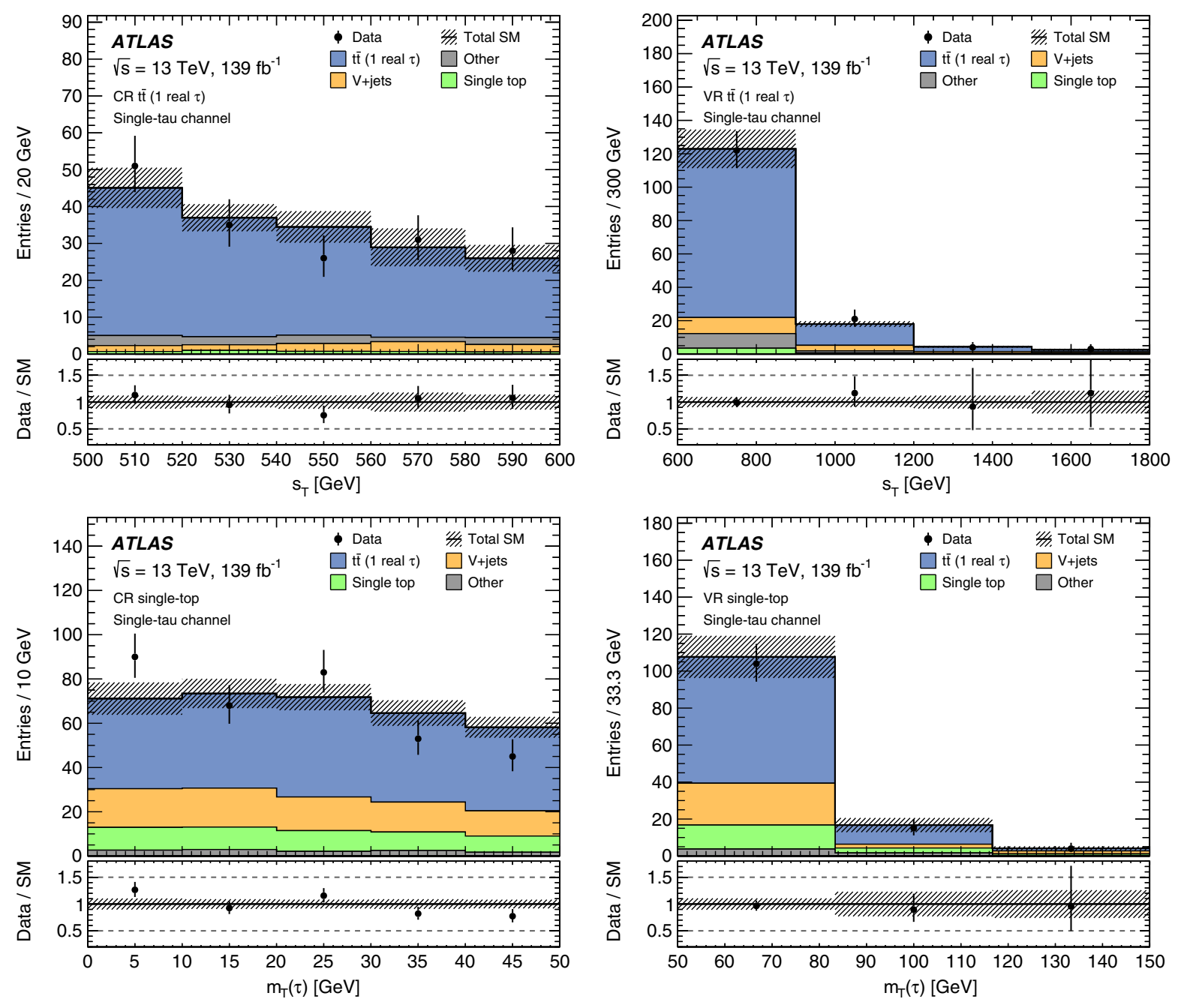

FIG. 5. Distributions of $s_{\mathrm{T}}$ and $m_{\mathrm{T}}(\tau)$ in the control regions and the validation regions of the single-tau channel. The top row shows $s_{\mathrm{T}}$ in the $t \bar{t}(1$ real $\tau) \mathrm{CR}$ (left) and $\bar{t}$ (1 real $\tau$ ) VR (right), the bottom row shows $m_{\mathrm{T}}(\tau)$ in the single-top CR (left) and single-top VR (right). The stacked histograms show the various SM background contributions. The hatched band indicates the total statistical and systematic uncertainty of the SM background. The $t \bar{t}(1$ real $\tau)$ and single-top background contributions are scaled with the normalization factors obtained from the background-only fit. Minor backgrounds are grouped together and denoted by "Other". This includes $t \bar{t}$-fake, $t \bar{t}+X$, multiboson, and other top. The rightmost bin includes the overflow in the upper right plot. 
parameters with Gaussian probability densities in the likelihood fits.

Experimental uncertainties comprise systematic uncertainties in the reconstruction, identification, calibration and corrections performed for the physics objects used in the analysis. Energy resolution and calibration uncertainties apply to all objects. For tau leptons, additional experimental systematic uncertainties arise from the reconstruction and identification efficiencies. Since events with prompt electrons and muons are rejected at preselection level, the related uncertainties in the reconstruction and identification are negligible in the analysis regions. For jets, additional uncertainties from the pileup subtraction, pseudorapidity intercalibration, flavor composition, and punch-through effects, as well as uncertainties in the flavor-tagging and jet-vertex tagging efficiencies, are considered. Systematic uncertainties affecting the energy or momentum of calibrated objects are propagated to the $E_{\mathrm{T}}^{\text {miss }}$ calculation, and an additional uncertainty due to the contribution of the soft-track term is considered. To test the robustness of the analysis against a potential mismodeling of events with two fake tau leptons, it was verified that an additional uncertainty of $100 \%$ in the $t \bar{t}$-fake background leads to a negligible decrease in the exclusion reach for the stop-stau signal model. Common sources of experimental uncertainty are assumed to be correlated across all regions and between the background processes and the signal.

Uncertainties in the renormalization and factorization scales are considered for all major background processes by separately varying the scales $\mu_{\mathrm{r}}$ and $\mu_{\mathrm{f}}$ up and down by a factor of 2 . Additionally, PDF and $\alpha_{\mathrm{s}}$ uncertainties are considered by following the PDF4LHC15 prescription [144]. The PDF uncertainty is evaluated as the root mean square of a set of 100 variations, and the effect of the $\alpha_{\mathrm{S}}$ uncertainty is derived by taking the average difference between the up and down variations. Additional initial-state and final-state radiation uncertainties are considered for the $t \bar{t}$ and single-top processes by varying generator settings, such as the simultaneous $\mu_{\mathrm{r}}$ and $\mu_{\mathrm{f}}$ variation and eigenvariations of the A14 tune [83]. Furthermore, theoretical uncertainties due to the hard-scatter and partonshower simulation are estimated by comparing the corresponding nominal yields against those predicted with alternative generators, i.e., PowHEg versus aMC@NLO and Pythia8 versus HeRWig7, respectively. The impact of the interference between the single-top $W t$ and $t \bar{t}$ production processes is estimated by comparing samples produced with the nominal diagram-removal scheme with alternative samples generated with the diagram-subtraction scheme [145]. For $V+$ jets, additional uncertainties related to the resummation and CKKW matching scales [146,147] are considered. Uncertainties in the cross section and in the integrated luminosity of the data are applied for all simulated processes except for $t \bar{t}$ with one or two real tau leptons and single top-quark production, which are
TABLE V. Relative systematic uncertainties in the estimated number of background events in the signal regions. In the lower part of the table, a breakdown of the total uncertainty into different categories is given. For the multibin SR, the breakdown refers to the integral over all three $p_{\mathrm{T}}(\tau)$ bins. Since the individual uncertainties are correlated, they do not sum in quadrature to equal the total background uncertainty.

\begin{tabular}{lccc}
\hline \hline Systematic uncertainty & \multirow{2}{*}{ Di-tau SR } & \multicolumn{2}{c}{ Single-tau } \\
\cline { 3 - 4 } & & one-bin SR & multibin SR \\
\hline Total & $25 \%$ & $17 \%$ & $17 \%$ \\
Jet-related & $19 \%$ & $4.2 \%$ & $3.9 \%$ \\
Tau-related & $4.7 \%$ & $5.5 \%$ & $4.3 \%$ \\
Other experimental & $3.7 \%$ & $1.0 \%$ & $0.8 \%$ \\
Theoretical modeling & $13 \%$ & $17 \%$ & $19 \%$ \\
MC statistics & $12 \%$ & $7.5 \%$ & $4.4 \%$ \\
Normalization factors & $8.8 \%$ & $15 \%$ & $16 \%$ \\
Luminosity & $0.8 \%$ & $0.5 \%$ & $0.4 \%$ \\
\hline \hline
\end{tabular}

normalized to data. The theoretical systematic uncertainties are assumed to be correlated across analysis regions and uncorrelated between all simulated processes.

Table V summarizes the total systematic uncertainties in the background expectation in the signal regions. In the di-tau SR the largest sources of experimental uncertainty are the uncertainties in the jet energy resolution, whereas hardscatter and parton-shower uncertainties dominate the uncertainty in the theoretical modeling. For the one-bin and multibin SRs in the single-tau channel, the theoretical uncertainties in $t \bar{t}$ event final-state radiation and in interference between the $t \bar{t}$ and $W t$ processes take the leading role.

For the theoretical uncertainties in the signal acceptance an estimate of $20 \%$ is used, which is derived from a study of the impact of varying the renormalization and factorization scales, the radiation and merging scales, the PDF, and the $\alpha_{\mathrm{s}}$ value for several stop-stau, $\mathrm{LQ}_{3}^{\mathrm{u} / \mathrm{d}}$, and $\mathrm{LQ}_{3}^{\mathrm{v}}$ signal points. Uncertainties in the signal production cross section are considered separately in the interpretation of the results discussed in Sec. VIII.

\section{RESULTS}

The predictions of the event yields from SM background processes obtained from the background-only fit to the control regions, as described in Sec. VI, and the observed data are shown in Table VI for the signal regions in the di-tau and single-tau channels. Events with pair-produced top quarks make up the largest contribution in all signal regions. The normalization factors obtained from the backgroundonly fit are $0.93_{-0.23}^{+0.32}$ for the $t \bar{t}$ ( 2 real $\tau$ ) background, $0.84_{-0.17}^{+0.21}$ for $t \bar{t}\left(1\right.$ real $\tau$ ), and $0.18_{-0.16}^{+0.19}$ for single-top production. The normalization factor for single-top production is significantly smaller than one and strongly depends on how the interference between single-top production at 
TABLE VI. Observed event yields in data ("observed") and expected event yields for SM background processes obtained from the background-only fit ("total bkg." and rows below) in the signal regions of the di-tau and single-tau channels. The quoted uncertainties include both the statistical and systematic uncertainties and are truncated at zero yield. By construction, no $t \bar{t}(2$ real $\tau$ ) events can pass the selections in the single-tau channel. Since the individual uncertainties are correlated, they do not sum in quadrature to equal the total background uncertainty.

\begin{tabular}{|c|c|c|c|c|c|}
\hline & \multirow[t]{2}{*}{ Di-tau SR } & \multirow[t]{2}{*}{ Single-tau SR (one-bin) } & \multicolumn{3}{|c|}{ Single-tau SR (binned in $p_{\mathrm{T}}(\tau)$ ) } \\
\hline & & & {$[50,100] \mathrm{GeV}$} & {$[100,200] \mathrm{GeV}$} & $>200 \mathrm{GeV}$ \\
\hline Observed & 2 & 6 & 8 & 6 & 2 \\
\hline Total bkg. & $4.1 \pm 1.0$ & $3.23 \pm 0.55$ & $10.1 \pm 1.8$ & $5.1 \pm 1.1$ & $2.05 \pm 0.64$ \\
\hline$t \bar{t}(2$ real $\tau)$ & $0.81 \pm 0.71$ & $\cdots$ & $\cdots$ & $\cdots$ & $\cdots$ \\
\hline$t \bar{t}(1$ real $\tau)$ & $0.82 \pm 0.27$ & $1.20 \pm 0.30$ & $4.8 \pm 1.2$ & $2.69 \pm 0.88$ & $0.64 \pm 0.29$ \\
\hline$t \bar{t}$-fake & $0.51 \pm 0.15$ & $0.69 \pm 0.15$ & $2.83 \pm 0.87$ & $0.66 \pm 0.17$ & $0.185 \pm 0.072$ \\
\hline Single top & $0.03_{-0.03}^{+0.10}$ & $0.39_{-0.39}^{+0.45}$ & $0.85_{-0.85}^{+0.86}$ & $0.54 \pm 0.54$ & $0.57 \pm 0.56$ \\
\hline$W+$ jets & $0.08_{-0.08}^{+0.11}$ & $0.35 \pm 0.16$ & $0.34 \pm 0.12$ & $0.64 \pm 0.24$ & $0.37 \pm 0.12$ \\
\hline$Z+$ jets & $0.35 \pm 0.14$ & $0.187 \pm 0.054$ & $0.275 \pm 0.081$ & $0.043 \pm 0.022$ & $0.123 \pm 0.048$ \\
\hline Multiboson & $0.48 \pm 0.21$ & $0.085 \pm 0.037$ & $0.163 \pm 0.037$ & $0.111 \pm 0.030$ & $0.030_{-0.030}^{+0.032}$ \\
\hline$t \bar{t}+V$ & $0.60 \pm 0.15$ & $0.242 \pm 0.064$ & $0.65 \pm 0.16$ & $0.31 \pm 0.12$ & $0.092 \pm 0.035$ \\
\hline$t \bar{t}+H$ & $0.28_{-0.28}^{+0.29}$ & $0.039_{-0.039}^{+0.040}$ & $0.10 \pm 0.10$ & $0.060_{-0.060}^{+0.061}$ & $0.028_{-0.028}^{+0.029}$ \\
\hline Other top & $0.122 \pm 0.067$ & $0.043 \pm 0.022$ & $0.096 \pm 0.074$ & $0.091 \pm 0.049$ & $0.0120 \pm 0.0084$ \\
\hline
\end{tabular}

next-to-leading order and leading-order $t \bar{t}$ production is handled $[145,148,149]$. The value 0.18 is obtained from the samples generated with the nominal diagram-removal scheme. The alternative diagram-subtraction scheme gives a normalization factor larger than 1 with very large uncertainties due to the much smaller yields and thus insufficient purity in the control region. The difference between the CR yields can be attributed to the much softer $b$-jet distribution for the diagram-subtraction scheme. However, the distribution shape of $m_{\mathrm{T}}\left(\tau_{1}\right)$, the variable used in the extrapolation from the control region to the signal region in the single-tau channel, agrees very well between the two schemes, giving confidence in the validity of the extrapolation. Furthermore, the predicted yields in the signal regions after the fit do not differ significantly between the two interference schemes, and the difference is taken into account as a systematic uncertainty.

No significant excess of data events above the SM expectation is observed in any of the signal regions. The largest excursions from the expected yields are a deficit with a significance of $1.0 \sigma$ in the signal region of the di-tau channel and an excess with a significance of $1.3 \sigma$ in the one-bin signal region of the single-tau channel, computed with the approximate formulas from Ref. [140]. The excess is not present, however, in the binned signal region of the single-tau channel. Figure 6 compares the observed data yields with the expected backgrounds for all event selections of the analysis. The entries in the rightmost column of the plot are the sum of the three bins of the multibin signal region in the single-tau channel, labeled "SR (multibin)." Figure 7 shows distributions of several kinematic variables for the expected SM background, and compares them with the distributions expected for several benchmark signal models and the observed data in the di-tau and single-tau signal regions.

In the absence of a significant excess, the analysis results are interpreted in terms of exclusion limits on the parameters of the stop-stau and leptoquark signal models. The limits are derived from a model-dependent fit, which includes the relevant signal regions in addition to the control regions, and signal contributions are taken into account in all analysis regions. The signal contamination of the control and validation regions does not exceed $10 \%$ (12\%) for model parameters that were not excluded by previous searches for the stop-stau (scalar leptoquark) signal. As there are no previous results for the vectorleptoquark model, the low $m\left(\mathrm{LQ}_{3}^{\mathrm{v}}\right)$ range is included in the interpretation, where sizable signal contributions to the control and validation regions can be present, exceeding $10 \%$ below 1100 (900) GeV for the Yang-Mills (minimalcoupling) scenario. However, as any signal contamination of the control regions is accounted for in the modeldependent fit, and none of the normalization factors are found to be larger than one, the signal contamination is not expected to weaken the interpretation for these cases either. Whether the signal-plus-background hypothesis is compatible with the observed event yields is assessed using the $\mathrm{CL}_{\mathrm{s}}$ prescription [150], for which the $p$-values are computed with asymptotic formulas obtained for a profilelikelihood ratio as the test statistic [140]. The validity of the asymptotic formulas has been checked through a comparison with the results from pseudoexperiments in the case of the model-independent limits. The likelihood is the product of Poisson terms modeling the joint probability of the event 


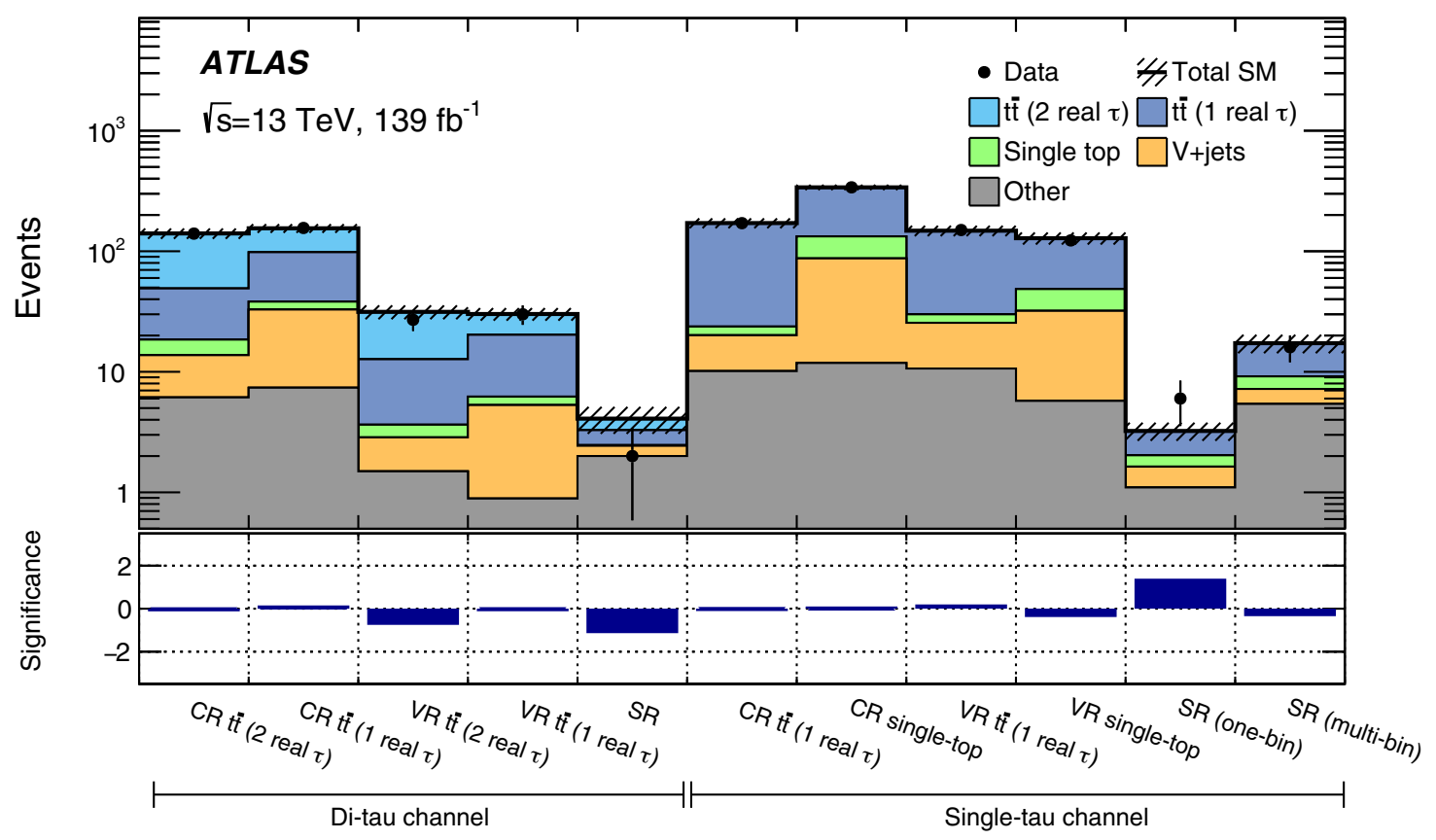

FIG. 6. Comparison of expected and observed event yields (top panel) and the significance of their difference (bottom panel) for all analysis regions of the di-tau and single-tau channels. The hatched band in the top panel indicates the combined statistical and systematic uncertainties in the expected SM background. The $t \bar{t}$ ( 2 real $\tau$ ) and $t \bar{t}$ ( 1 real $\tau$ ) contributions and the single-top background contributions are scaled with the normalization factors obtained from the background-only fit. Minor backgrounds are grouped together and denoted by "Other". This includes $t \bar{t}$-fake, $t \bar{t}+X$, multiboson, and other top. The entries in the column labeled "SR (multibin)" are the sum of the three bins of the multibin signal region. The significance is computed following Eq. (25) from Ref. [151], multiplied by -1 if the observed number of events is smaller than the expected background yield.

yields for all analysis regions considered in the fit and Gaussian probability terms that constrain the nuisance parameters related to the systematic uncertainties. Figure 8 shows the expected and observed exclusion contours at the 95\% confidence level for the stop-stau signal computed from the model-dependent fit that includes both the di-tau and single-tau multibin SR. All systematic uncertainties are included in the fit with the exception of the signal crosssection uncertainty, for which a separate band around the observed limit contour is drawn instead. The expected exclusion reach of the analysis extends to top-squark masses around 1.35 TeVover a wide range of tau-slepton masses, and to tau-slepton masses around $1.15 \mathrm{TeV}$. With decreasing tauslepton mass, most noticeably below $400 \mathrm{GeV}$, the exclusion reach in top-squark mass becomes lower, because the fraction of $E_{\mathrm{T}}^{\text {miss }}$ that is due to the neutrinos from the topsquark decay increases, and thus the discrimination power of $m_{\mathrm{T} 2}$ is reduced. The observed exclusion reach slightly exceeds the expected exclusion reach, as the sensitivity to the stop-stau signal model for tau-slepton masses larger than $200 \mathrm{GeV}$ is dominated by the di-tau SR with an observed deficit. Top-squark masses of up to $1.4 \mathrm{TeV}$ and tau-slepton masses of up to $1.2 \mathrm{TeV}$ are excluded at the $95 \%$ confidence level in this specific model. These are the strongest mass limits for these two supersymmetric particles in a simplified model from run 2 of the LHC to date. They extend significantly beyond the limits from the previous ATLAS analysis, which are shown in the plot for comparison. The gain in sensitivity is partly due to the larger dataset used in the analysis, but also due to improved reconstruction and identification algorithms for tau leptons and $b$-jets, and an improved signal-region strategy with reoptimized selection requirements and the added single-tau signal region targeting low tau-slepton masses.

Exclusion limits for the scalar-leptoquark signal are shown in the two plots in Fig. 9, where the upper plot considers pair production of up-type leptoquarks $\mathrm{LQ}_{3}^{\mathrm{u}}$ and the bottom plot pair production of down-type leptoquarks $\mathrm{LQ}_{3}^{\mathrm{d}}$. To derive these exclusion limits, the model-dependent fit includes, besides the four CRs, only the single-tau multibin SR. The di-tau SR has not been optimized for the leptoquark models, as final states with two tau leptons are covered by a previous search [63]. It has been checked that as a consequence this SR does not significantly contribute to the exclusion sensitivity, and it is thus not included in the interpretation for the leptoquark models. For both types of scalar leptoquarks, the expected and observed exclusion contours extend to masses around $1.25 \mathrm{TeV}$ at the $95 \%$ confidence level for intermediate values of the branching fraction $B\left(\mathrm{LQ}_{3}^{\mathrm{u} / \mathrm{d}} \rightarrow q \ell\right)$. When $B\left(\mathrm{LQ}_{3}^{\mathrm{u} / \mathrm{d}} \rightarrow q \ell\right)$ approaches zero or one, the fraction of events with exactly one tau lepton decreases accordingly, leading to a reduction of the 

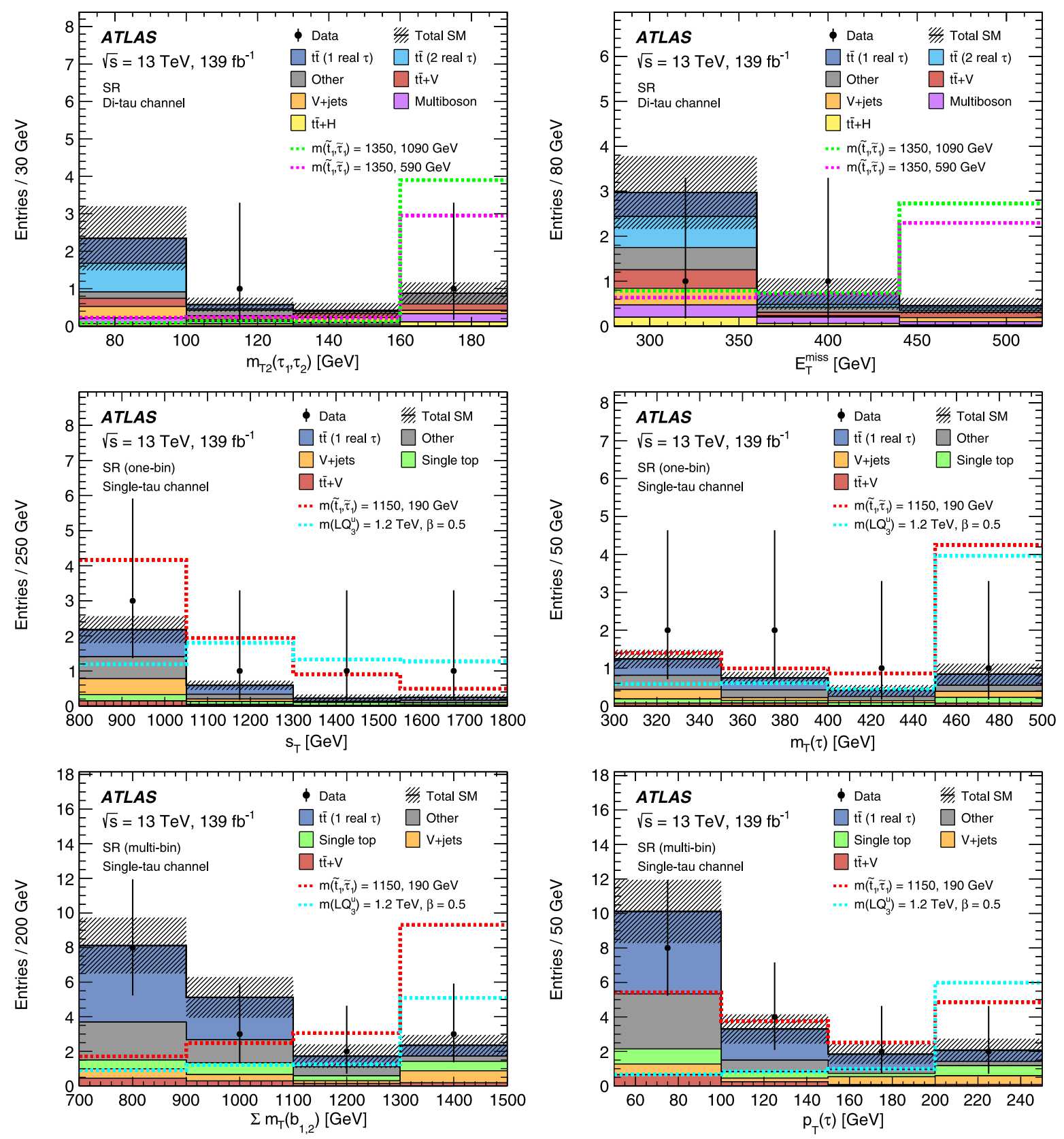

FIG. 7. Distributions of $m_{\mathrm{T} 2}\left(\tau_{1}, \tau_{2}\right)$ and $E_{\mathrm{T}}^{\mathrm{miss}}$ in the di-tau SR (top), of $s_{\mathrm{T}}$ and $m_{\mathrm{T}}(\tau)$ in the single-tau one-bin SR (middle) and of $\sum m_{\mathrm{T}}\left(b_{1,2}\right)$ and $p_{\mathrm{T}}(\tau)$ in the single-tau $p_{\mathrm{T}}(\tau)$-binned SR (bottom). The stacked histograms show the various SM background contributions. The hatched band indicates the total statistical and systematic uncertainty of the SM background. The $t \bar{t}(2$ real $\tau$ ) and $t \bar{t}(1$ real $\tau$ ) contributions and the single-top background contributions are scaled with the normalization factors obtained from the background-only fit. Minor backgrounds are grouped together and denoted by "Other". This includes $t \bar{t}$-fake, single top, and other top (di-tau channel) or $t \bar{t}$-fake, $t \bar{t}+H$, multiboson, and other top (single-tau channel). The overlaid dotted lines show the additional contributions for signal scenarios close to the expected exclusion contour with the particle type and the mass and $\beta$ parameters for the simplified models indicated in the legend. For the leptoquark signal model, the shapes of the distributions for $\mathrm{LQ}_{3}^{\mathrm{d}}$ and $\mathrm{LQ}_{3}^{\mathrm{v}}$ (not shown) are similar to that of $\mathrm{LQ}_{3}^{\mathrm{u}}$. The rightmost bin includes the overflow.

signal acceptance and thus a lower mass reach. At $B\left(\mathrm{LQ}_{3}^{\mathrm{u} / \mathrm{d}} \rightarrow q \ell\right)=0$ the leptoquark decays do not directly produce any tau leptons. The signal events only pass the signal-region selection if a top quark from an up-type leptoquark decay produces a tau lepton or if a fake tau lepton is present in the event. This leads to a large decrease in mass reach. The reduction of the probability of signal events to pass the SR selection when approaching $B\left(\mathrm{LQ}_{3}^{\mathrm{u} / \mathrm{d}} \rightarrow q \ell\right)=0$ is compensated by the cross section. For $m(\mathrm{LQ})=750 \mathrm{GeV}$, corresponding to the mass reach at $B\left(\mathrm{LQ}_{3}^{\mathrm{u} / \mathrm{d}} \rightarrow q \ell\right)=0$, the 


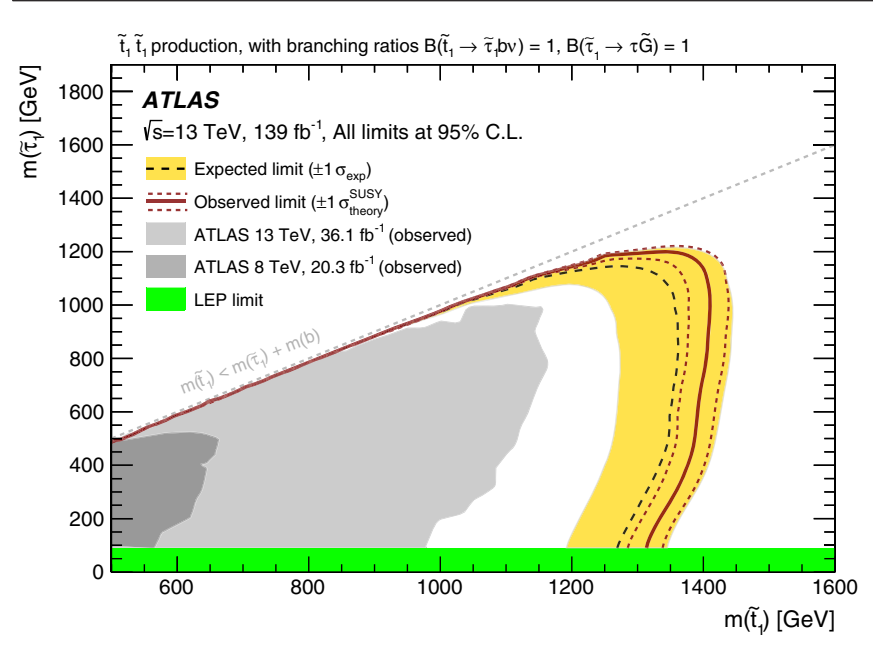

FIG. 8. Exclusion contours at the 95\% confidence level (C.L.) for the stop-stau signal model as a function of the masses of the top squark $m(\tilde{t})$ and the tau slepton $m(\tilde{\tau})$. Expected and observed limits are shown for the present search in comparison with observed limits from previous ATLAS analyses based on data from run 1 of the LHC at $\sqrt{s}=8 \mathrm{TeV}$ [57] and on a partial dataset from run 2 at $\sqrt{s}=13 \mathrm{TeV}$ [58]. The green band indicates the lower limit on the mass of the tau slepton (for a massless LSP) from the LEP experiments [152].

cross section is larger by a factor of about 40 compared to the cross section at $m(\mathrm{LQ})=1250 \mathrm{GeV}$, corresponding to the excluded LQ mass at $B\left(\mathrm{LQ}_{3}^{\mathrm{u} / \mathrm{d}} \rightarrow q \ell\right)=0.5$.

The exclusion contours from the interpretation of the analysis results for the vector-leptoquark models are shown in the two plots in Fig. 10. As in the scalar-leptoquark case, the model-dependent fit includes, besides the four CRs, only the single-tau multibin SR. For intermediate values of the branching fraction $B\left(\mathrm{LQ}_{3}^{\mathrm{v}} \rightarrow b \tau\right)$, the expected and observed exclusion contours at the $95 \%$ confidence level extend to masses around $1.5 \mathrm{TeV}$ in the minimal-coupling scenario and to masses around $1.8 \mathrm{TeV}$ in the Yang-Mills scenario. As expected, the shape of the contours as a function of $B\left(\mathrm{LQ}_{3}^{\mathrm{v}} \rightarrow b \tau\right)$ is very similar to that for the scalar-leptoquark models, and the larger cross sections for the pair production of vector leptoquarks lead to a larger mass reach.

In addition to the model-dependent interpretations for the signal models shown above, model-independent statements about the presence of physics that is not included in the background expectation for SM processes can also be derived from the analysis results. The model-independent fit is performed for each of the one-bin SRs of the two analysis channels separately. As no specific model is assumed, the contamination of the CRs by a potential signal is neglected, and a generic signal of variable strength is included in the SR. Table VII states the observed and expected upper limits, $S_{\mathrm{obs}}^{95}$ and $S_{\mathrm{exp}}^{95}$, on the number of signal events at the $95 \%$ confidence level based on the $\mathrm{CL}_{\mathrm{s}}$
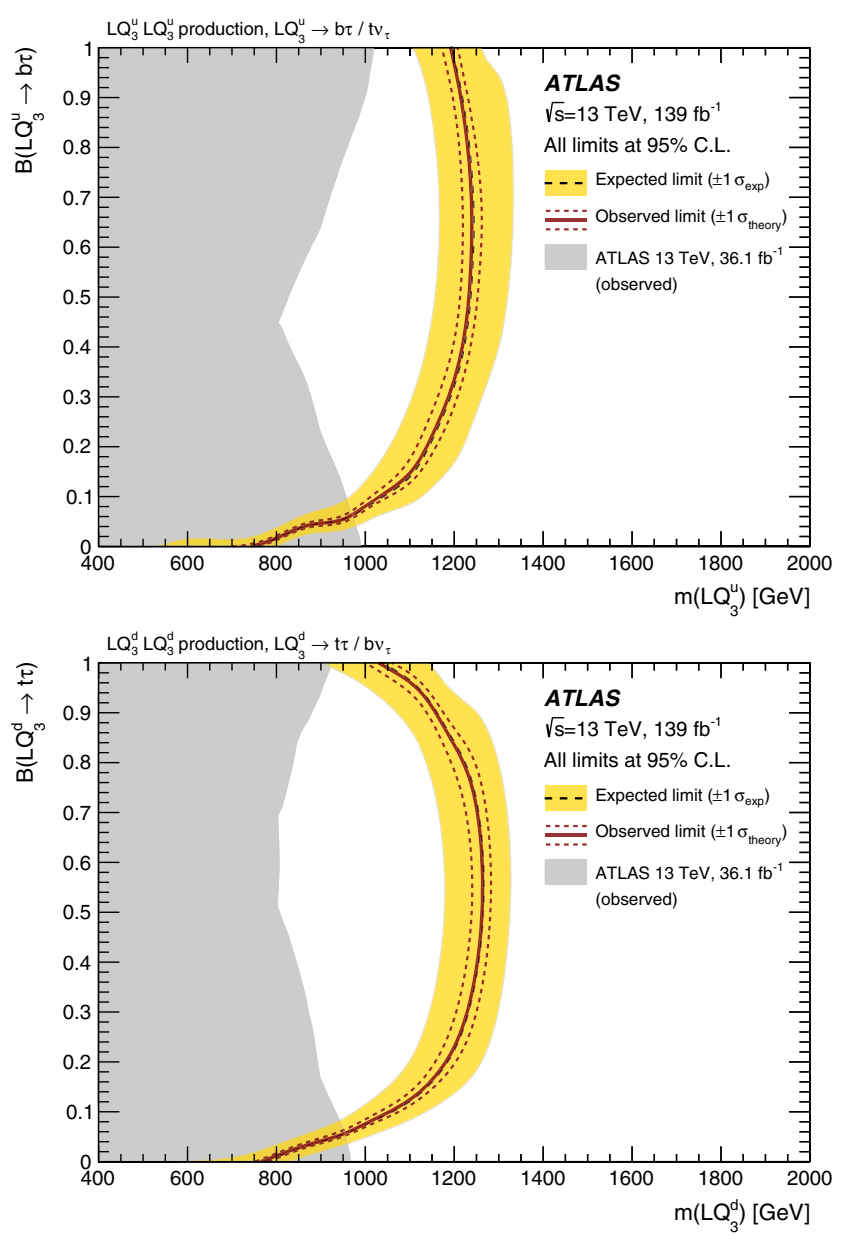

FIG. 9. Expected and observed exclusion contours at the 95\% confidence level (C.L.) for the third-generation scalarleptoquark signal model, as a function of the mass $m\left(\mathrm{LQ}_{3}^{\mathrm{u} / \mathrm{d}}\right)$ and the branching fraction $B\left(\mathrm{LQ}_{3}^{\mathrm{u} / \mathrm{d}} \rightarrow q \ell\right)$ into a quark and a charged lepton. The top plot shows the exclusion contour for uptype leptoquarks $\mathrm{LQ}_{3}^{\mathrm{u}}$ with charge $+\frac{2}{3} e$, the bottom plot the exclusion contour for down-type leptoquarks $\mathrm{LQ}_{3}^{\mathrm{d}}$ with charge $-\frac{1}{3} e$. The limits are derived from the binned single-tau signal region. Shown in gray for comparison are the observed exclusionlimit contours from the previous ATLAS publication that targets the same leptoquark models but is based on a subset of the run-2 data [61]. In that publication, five different analyses were considered that target not only the final state studied here but also the final states that correspond to a branching fraction $B\left(\mathrm{LQ}_{3}^{\mathrm{u} / \mathrm{d}} \rightarrow q \ell\right)$ of 0 or 1 , leading to the concave shapes of the gray exclusion contours.

prescription, where the test statistic is evaluated using pseudoexperiments. These upper limits are also expressed as upper limits on the visible signal cross section $\langle A \epsilon \sigma\rangle_{\text {obs }}^{95}$, which is defined as the product of acceptance $A$, reconstruction efficiency $\epsilon$ and signal cross section $\sigma$. The table also reports the $\mathrm{CL}_{\mathrm{b}}$ value, i.e., the confidence level observed for the background-only hypothesis, the discovery $p$-value, defined as the probability to find 

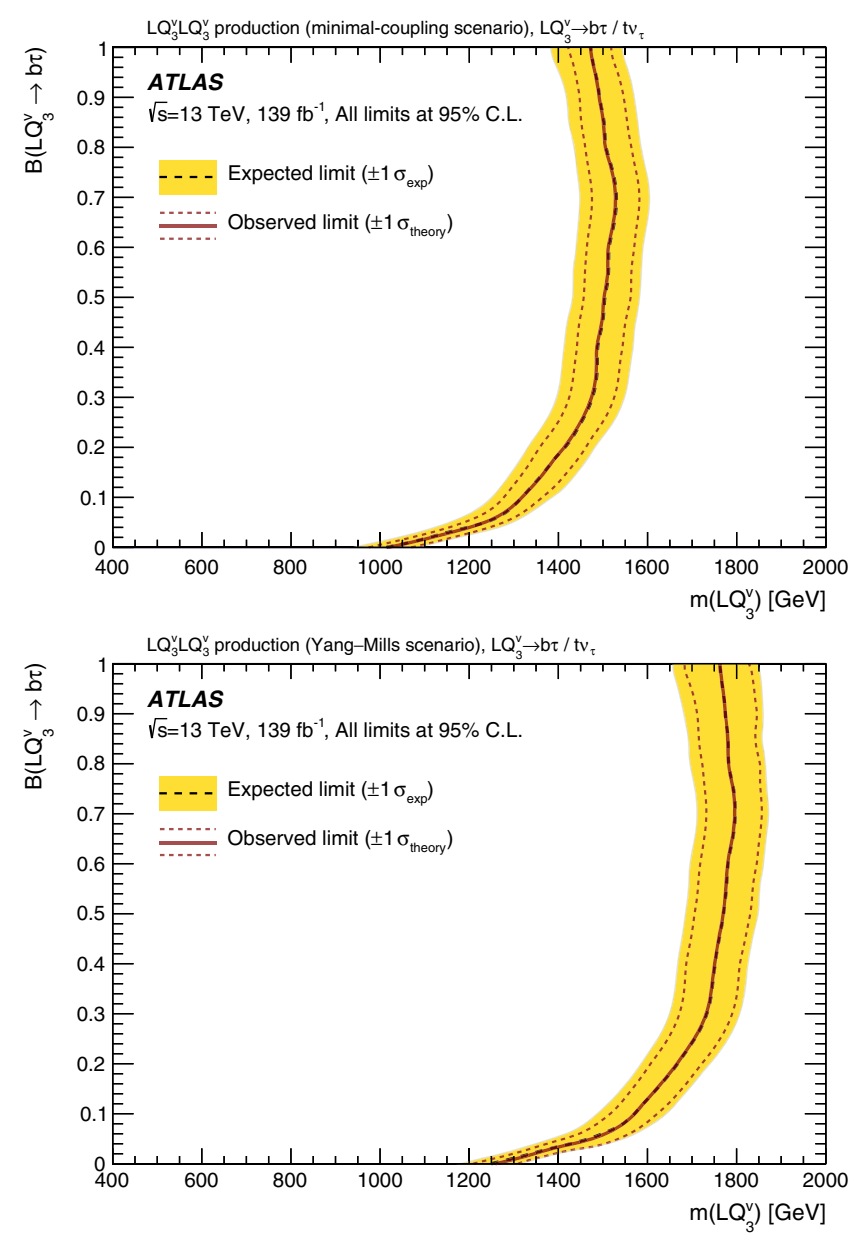

FIG. 10. Expected and observed exclusion contours at the 95\% confidence level (C.L.) for the third-generation vectorleptoquark signal model, as a function of the mass $m\left(\mathrm{LQ}_{3}^{\mathrm{v}}\right)$ and the branching fraction $B\left(\mathrm{LQ}_{3}^{\mathrm{v}} \rightarrow b \tau\right)$ into a quark and a charged lepton. The top plot shows the exclusion contour for the minimalcoupling scenario, the bottom plot the exclusion contour for vector leptoquarks in the Yang-Mills scenario. The limits are derived from the binned single-tau signal region.

the observed number of events or more under the background-only hypothesis, and the equivalent significance for each of the two channels.

\section{CONCLUSION}

In this paper, a search for new phenomena in final states with hadronically decaying tau leptons, $b$-jets and large missing transverse momentum is presented. This signature provides sensitivity to models in which the new particles preferentially decay into third-generation Standard Model particles. The analysis exploits the full dataset recorded with the ATLAS detector in run 2 of the LHC, corresponding to $139 \mathrm{fb}^{-1}$ of proton-proton collisions at $\sqrt{s}=13 \mathrm{TeV}$. No significant excess of events is observed over the Standard Model expectation. The results are thus interpreted in terms of exclusion limits at $95 \%$ confidence level for two simplified models with pair production of supersymmetric top squarks or leptoquarks which are assumed to only decay into thirdgeneration fermions. In the case of the supersymmetric model, masses up to $1.4 \mathrm{TeV}$ are excluded for top squarks decaying via tau sleptons into nearly massless gravitinos across a wide range of tau-slepton masses. For both up-type and down-type scalar leptoquarks, masses up to about $1.25 \mathrm{TeV}$ can be excluded. For vector leptoquarks with minimal couplings, masses up to about $1.5 \mathrm{TeV}$ can be excluded, and up to about $1.8 \mathrm{TeV}$ for vector leptoquarks with additional couplings to gauge bosons. The larger dataset, updated reconstruction and identification algorithms for tau leptons and $b$-jets, and the optimized analysis strategy yield significantly better sensitivity than in earlier LHC studies. Based on the considered benchmark models, the search yields the strongest mass limits to date on pairproduced top squarks and on pair-produced third-generation scalar and vector leptoquarks at intermediate values of the branching fraction into a quark and a charged lepton.

\section{ACKNOWLEDGMENTS}

We thank CERN for the very successful operation of the LHC, as well as the support staff from our institutions without whom ATLAS could not be operated efficiently. We acknowledge the support of ANPCyT, Argentina; YerPhI, Armenia; ARC, Australia; BMWFW and FWF, Austria; ANAS, Azerbaijan; SSTC, Belarus; CNPq and FAPESP, Brazil; NSERC, NRC and CFI, Canada; CERN; ANID, Chile; CAS, MOST and NSFC, China; Minciencias, Colombia; MSMT CR, MPO CR and VSC CR, Czech

TABLE VII. From left to right: upper limits at the $95 \%$ confidence level (C.L.) on the visible cross section $\left(\langle A \epsilon \sigma\rangle_{\mathrm{obs}}^{95}\right)$ and on the number of signal events $\left(S_{\mathrm{obs}}^{95}\right)$. The third column $\left(S_{\mathrm{exp}}^{95}\right)$ shows the upper limit at the $95 \%$ C.L. on the number of signal events, given the expected number (and $\pm 1 \sigma$ excursions of the expectation) of background events. The last two columns indicate the confidence level observed for the background-only hypothesis $\left(\mathrm{CL}_{\mathrm{b}}\right)$, the discovery $p$-value $(p(s=0))$ and the significance $(Z)$. In the di-tau $\mathrm{SR}$, where fewer events are observed than predicted by the fitted background estimate, the $p$-value is capped at 0.5 .

\begin{tabular}{lcccccc}
\hline \hline Analysis region & $\langle A \epsilon \sigma\rangle_{\mathrm{obs}}^{95}[\mathrm{fb}]$ & $S_{\mathrm{obs}}^{95}$ & $S_{\mathrm{exp}}^{95}$ & $\mathrm{CL}_{\mathrm{b}}$ & $p(s=0)$ & $Z$ \\
\hline Di-tau SR & 0.03 & 4.1 & $5.3_{-1.5}^{+2.2}$ & 0.18 & 0.50 & 0.0 \\
Single-tau one-bin SR & 0.06 & 8.2 & $5.1_{-1.3}^{+2.1}$ & 0.91 & 0.08 & 1.37 \\
\hline \hline
\end{tabular}


Republic; DNRF and DNSRC, Denmark; IN2P3-CNRS and CEA-DRF/IRFU, France; SRNSFG, Georgia; BMBF, HGF and MPG, Germany; GSRI, Greece; RGC and Hong Kong SAR, China; ISF and Benoziyo Center, Israel; INFN, Italy; MEXT and JSPS, Japan; CNRST, Morocco; NWO, Netherlands; RCN, Norway; MNiSW and NCN, Poland; FCT, Portugal; MNE/IFA, Romania; JINR; MES of Russia and NRC KI, Russian Federation; MESTD, Serbia; MSSR, Slovakia; ARRS and MIZŠ, Slovenia; DSI/NRF, South Africa; MICINN, Spain; SRC and Wallenberg Foundation, Sweden; SERI, SNSF and Cantons of Bern and Geneva, Switzerland; MOST, Taiwan; TAEK, Turkey; STFC, United Kingdom; DOE and NSF, United States of America. In addition, individual groups and members have received support from BCKDF, CANARIE, Compute Canada and CRC, Canada; COST, ERC, ERDF, Horizon 2020 and Marie Skłodowska-Curie Actions, European Union; Investissements d'Avenir Labex, Investissements d'Avenir Idex and ANR, France; DFG and AvH
Foundation, Germany; Herakleitos, Thales and Aristeia programmes co-financed by EU-ESF and the Greek NSRF, Greece; BSF-NSF and GIF, Israel; Norwegian Financial Mechanism 2014-2021, Norway; La Caixa Banking Foundation, CERCA Programme Generalitat de Catalunya and PROMETEO and GenT Programmes Generalitat Valenciana, Spain; Göran Gustafssons Stiftelse, Sweden; The Royal Society and Leverhulme Trust, United Kingdom. The crucial computing support from all WLCG partners is acknowledged gratefully, in particular from CERN, the ATLAS Tier-1 facilities at TRIUMF (Canada), NDGF (Denmark, Norway, Sweden), CC-IN2P3 (France), KIT/GridKA (Germany), INFN-CNAF (Italy), NL-T1 (Netherlands), PIC (Spain), ASGC (Taiwan), RAL (UK) and BNL (USA), the Tier-2 facilities worldwide and large non-WLCG resource providers. Major contributors of computing resources are listed in Ref. [153].
[1] Y. Golfand and E. Likhtman, Extension of the algebra of poincare group generators and violation of $\mathrm{P}$ invariance, Pis'ma Zh. Eksp. Teor. Fiz. 13, 452 (1971) [JETP Lett. 13, 323 (1971)], http://jetpletters.ru/ps/1584/article_24309 .pdf.

[2] D. Volkov and V. Akulov, Is the neutrino a goldstone particle?, Phys. Lett. 46B, 109 (1973).

[3] J. Wess and B. Zumino, Supergauge transformations in four dimensions, Nucl. Phys. B70, 39 (1974).

[4] J. Wess and B. Zumino, Supergauge invariant extension of quantum electrodynamics, Nucl. Phys. B78, 1 (1974).

[5] S. Ferrara and B. Zumino, Supergauge invariant YangMills theories, Nucl. Phys. B79, 413 (1974).

[6] A. Salam and J. Strathdee, Super-symmetry and nonAbelian gauges, Phys. Lett. 51B, 353 (1974).

[7] S. P. Martin, A supersymmetry primer, Adv. Ser. Dir. High Energy Phys. 18, 1 (1998).

[8] N. Sakai, Naturalness in supersymmetric GUTS, Z. Phys. C 11, 153 (1981).

[9] S. Dimopoulos, S. Raby, and F. Wilczek, Supersymmetry and the scale of unification, Phys. Rev. D 24, 1681 (1981).

[10] L. E. Ibáñez and G. G. Ross, Low-energy predictions in supersymmetric grand unified theories, Phys. Lett. 105B, 439 (1981).

[11] S. Dimopoulos and H. Georgi, Softly broken supersymmetry and SU(5), Nucl. Phys. B193, 150 (1981).

[12] G. R. Farrar and P. Fayet, Phenomenology of the production, decay, and detection of new hadronic states associated with supersymmetry, Phys. Lett. 76B, 575 (1978).

[13] H. Goldberg, Constraint on the Photino Mass from Cosmology, Phys. Rev. Lett. 50, 1419 (1983); 103, 099905(E) (2009).
[14] J. Ellis, J. Hagelin, D. V. Nanopoulos, K. A. Olive, and M. Srednicki, Supersymmetric relics from the big bang, Nucl. Phys. B238, 453 (1984).

[15] W. De Boer, Grand unified theories and supersymmetry in particle physics and cosmology, Prog. Part. Nucl. Phys. 33, 201 (1994).

[16] H. Georgi and S. Glashow, Unity of All ElementaryParticle Forces, Phys. Rev. Lett. 32, 438 (1974).

[17] E. Farhi and L. Susskind, Technicolour, Phys. Rep. 74, 277 (1981).

[18] B. Schrempp and F. Schrempp, Light leptoquarks, Phys. Lett. 153B, 101 (1985).

[19] V. D. Angelopoulos, J. Ellis, H. Kowalski, D. V. Nanopoulos, N. D. Tracas, and F. Zwirner, Search for new quarks suggested by the superstring, Nucl. Phys. B292, 59 (1987).

[20] W. Buchmüller and D. Wyler, Constraints on SU(5)-type leptoquarks, Phys. Lett. B 177, 377 (1986).

[21] R. Barbier et al., R-parity-violating supersymmetry, Phys. Rep. 420, 1 (2005).

[22] G. Hiller and M. Schmaltz, $R_{K}$ and future $b \rightarrow s \ell \ell$ physics beyond the standard model opportunities, Phys. Rev. D 90 , 054014 (2014).

[23] B. Gripaios, M. Nardecchia, and S. A. Renner, Composite leptoquarks and anomalies in $B$-meson decays, J. High Energy Phys. 05 (2015) 006.

[24] M. Freytsis, Z. Ligeti, and J. T. Ruderman, Flavor models for $\bar{B} \rightarrow D^{(*)} \tau \bar{\nu}$, Phys. Rev. D 92, 054018 (2015).

[25] M. Bauer and M. Neubert, Minimal Leptoquark Explanation for the $R_{D^{(*)}}, R_{K}$, and $(g-2)_{\mu}$ Anomalies, Phys. Rev. Lett. 116, 141802 (2016). 
[26] L. Di Luzio and M. Nardecchia, What is the scale of new physics behind the $B$-flavour anomalies?, Eur. Phys. J. C 77, 536 (2017).

[27] D. Buttazzo, A. Greljo, G. Isidori, and D. Marzocca, Bphysics anomalies: A guide to combined explanations, J. High Energy Phys. 11 (2017) 044.

[28] J. M. Cline, B decay anomalies and dark matter from vectorlike confinement, Phys. Rev. D 97, 015013 (2018).

[29] $B A B A R$ Collaboration, Measurement of an excess of $\bar{B} \rightarrow$ $D^{(*)} \tau^{-} \bar{\nu}_{\tau}$ decays and implications for charged Higgs bosons, Phys. Rev. D 88, 072012 (2013).

[30] Belle Collaboration, Measurement of the branching ratio of $\bar{B} \rightarrow D^{(*)} \tau^{-} \bar{\nu}_{\tau}$ relative to $\bar{B} \rightarrow D^{(*)} \ell^{-} \bar{\nu}_{\ell}$ decays with hadronic tagging at Belle, Phys. Rev. D 92, 072014 (2015).

[31] LHCb Collaboration, Measurement of the Ratio of Branching Fractions $\mathcal{B}\left(\bar{B}^{0} \rightarrow D^{*+} \tau^{-} \bar{\nu}_{\tau}\right) / \mathcal{B}\left(\bar{B}^{0} \rightarrow D^{*+} \mu^{-} \bar{\nu}_{\mu}\right)$, Phys. Rev. Lett. 115, 111803 (2015); 115, 159901(E) (2015).

[32] Belle Collaboration, Measurement of the $\tau$ Lepton Polarization and $R\left(D^{*}\right)$ in the Decay $\bar{B} \rightarrow D^{*} \tau^{-} \bar{\nu}_{\tau}$, Phys. Rev. Lett. 118, 211801 (2017).

[33] LHCb Collaboration, Measurement of the Ratio of Branching Fractions $\mathcal{B}\left(B_{c}^{+} \rightarrow J / \psi \tau^{+} \nu_{\tau}\right) / \mathcal{B}\left(B_{c}^{+} \rightarrow J / \psi \mu^{+} \nu_{\mu}\right)$, Phys. Rev. Lett. 120, 121801 (2018).

[34] LHCb Collaboration, Angular analysis of the $B^{0} \rightarrow$ $K^{* 0} \mu^{+} \mu^{-}$decay using $3 \mathrm{fb}^{-1}$ of integrated luminosity, J. High Energy Phys. 02 (2016) 104.

[35] Belle Collaboration, Lepton-Flavor-Dependent Angular Analysis of $B \rightarrow K^{*} \ell^{+} \ell^{-}$, Phys. Rev. Lett. 118, 111801 (2017).

[36] LHCb Collaboration, Test of lepton universality with $B^{0} \rightarrow$ $K^{* 0} \ell^{+} \ell^{-}$decays, J. High Energy Phys. 08 (2017) 055.

[37] LHCb Collaboration, Test of lepton universality in beautyquark decays, arXiv:2103.11769.

[38] E. C. Leskow, G. D’Ambrosio, A. Crivellin, and D. Müller, $(g-2)_{\mu}$, lepton flavor violation, and $Z$ decays with leptoquarks: Correlations and future prospects, Phys. Rev. D 95, 055018 (2017).

[39] D. Chakraverty, D. Choudhury, and A. Datta, A nonsupersymmetric resolution of the anomalous muon magnetic moment, Phys. Lett. B 506, 103 (2001).

[40] Muon g-2 Collaboration, Measurement of the Positive Muon Anomalous Magnetic Moment to 0.46 ppm, Phys. Rev. Lett. 126, 141801 (2021).

[41] M. Dine and W. Fischler, A phenomenological model of particle physics based on supersymmetry, Phys. Lett. 110B, 227 (1982).

[42] L. Alvarez-Gaumé, M. Claudson, and M. B. Wise, Lowenergy supersymmetry, Nucl. Phys. B207, 96 (1982).

[43] C. R. Nappi and B. A. Ovrut, Supersymmetric extension of the $\operatorname{SU}(3) \times \mathrm{SU}(2) \times \mathrm{U}(1)$ model, Phys. Lett. 113B, 175 (1982).

[44] M. Asano, H. D. Kim, R. Kitano, and Y. Shimizu, Natural supersymmetry at the LHC, J. High Energy Phys. 12 (2010) 019.

[45] K. Inoue, A. Kakuto, H. Komatsu, and S. Takeshita, Aspects of grand unified models with softly broken supersymmetry, Prog. Theor. Phys. 68, 927 (1982); 70, 330(E) (1983).

[46] J. R. Ellis and S. Rudaz, Search for supersymmetry in toponium decays, Phys. Lett. B 128, 248 (1983).
[47] J. Alwall, M.-P. Le, M. Lisanti, and J. G. Wacker, Searching for directly decaying gluinos at the Tevatron, Phys. Lett. B 666, 34 (2008).

[48] J. Alwall, P. Schuster, and N. Toro, Simplified models for a first characterization of new physics at the LHC, Phys. Rev. D 79, 075020 (2009).

[49] D. Alves et al., Simplified models for LHC new physics searches, J. Phys. G 39, 105005 (2012).

[50] ATLAS Collaboration, Search for a scalar partner of the top quark in the all-hadronic $t \bar{t}$ plus missing transverse momentum final state at $\sqrt{s}=13 \mathrm{TeV}$ with the ATLAS detector, Eur. Phys. J. C 80, 737 (2020).

[51] ATLAS Collaboration, Search for new phenomena with top quark pairs in final states with one lepton, jets, and missing transverse momentum in $p p$ collisions at $\sqrt{s}=$ $13 \mathrm{TeV}$ with the ATLAS detector, J. High Energy Phys. 04 (2021) 174.

[52] ATLAS Collaboration, Search for new phenomena in events with two opposite-charge leptons, jets and missing transverse momentum in $p p$ collisions at $\sqrt{s}=13 \mathrm{TeV}$ with the ATLAS detector, J. High Energy Phys. 04 (2021) 165.

[53] CMS Collaboration, Search for top squark production in fully-hadronic final states in proton-proton collisions at $\sqrt{s}=13 \mathrm{TeV}$, Phys. Rev. D 104, 052001 (2021).

[54] CMS Collaboration, Search for direct top squark pair production in events with one lepton, jets, and missing transverse momentum at $13 \mathrm{TeV}$ with the CMS experiment, J. High Energy Phys. 05 (2020) 032.

[55] CMS Collaboration, Search for top squark pair production using dilepton final states in $p p$ collision data collected at $\sqrt{s}=13 \mathrm{TeV}$, Eur. Phys. J. C 81, 3 (2021).

[56] CMS Collaboration, Combined searches for the production of supersymmetric top quark partners in proton-proton collisions at $\sqrt{s}=13 \mathrm{TeV}$, Eur. Phys. J. C 81, 970 (2021).

[57] ATLAS Collaboration, Search for direct top squark pair production in final states with two tau leptons in $p p$ collisions at $\sqrt{s}=8 \mathrm{TeV}$ with the ATLAS detector, Eur. Phys. J. C 76, 81 (2016).

[58] ATLAS Collaboration, Search for top squarks decaying to tau sleptons in $p p$ collisions at $\sqrt{s}=13 \mathrm{TeV}$ with the ATLAS detector, Phys. Rev. D 98, 032008 (2018).

[59] CMS Collaboration, Search for top squark pair production in a final state with two tau leptons in proton-proton collisions at $\sqrt{s}=13 \mathrm{TeV}$, J. High Energy Phys. 02 (2020) 015.

[60] W. Buchmüller, R. Rückl, and D. Wyler, Leptoquarks in lepton—quark collisions, Phys. Lett. B 191, 442 (1987); 448, 320(E) (1999).

[61] ATLAS Collaboration, Searches for third-generation scalar leptoquarks in $\sqrt{s}=13 \mathrm{TeV} p p$ collisions with the ATLAS detector, J. High Energy Phys. 06 (2019) 144.

[62] ATLAS Collaboration, Search for new phenomena in final states with $b$-jets and missing transverse momentum in $\sqrt{s}=13 \mathrm{TeV} p p$ collisions with the ATLAS detector, J. High Energy Phys. 05 (2021) 093.

[63] ATLAS Collaboration, Search for pair production of thirdgeneration scalar leptoquarks decaying into a top quark and a $\tau$-lepton in $p p$ collisions at $\sqrt{s}=13 \mathrm{TeV}$ with the ATLAS detector, J. High Energy Phys. 06 (2021) 179. 
[64] CMS Collaboration, Search for singly and pair-produced leptoquarks coupling to third-generation fermions in proton-proton collisions at $\sqrt{s}=13 \mathrm{TeV}$, Phys. Lett. B 819 , 136446 (2021).

[65] CMS Collaboration, Constraints on models of scalar and vector leptoquarks decaying to a quark and a neutrino at $\sqrt{s}=13$ TeV, Phys. Rev. D 98, 032005 (2018).

[66] CMS Collaboration, Search for third-generation scalar leptoquarks decaying to a top quark and a $\tau$ lepton at $\sqrt{s}=13$ TeV, Eur. Phys. J. C 78, 707 (2018).

[67] CMS Collaboration, Search for Leptoquarks Coupled to Third-Generation Quarks in Proton-Proton Collisions at $\sqrt{s}=13$ TeV, Phys. Rev. Lett. 121, 241802 (2018).

[68] CMS Collaboration, Search for heavy neutrinos and thirdgeneration leptoquarks in hadronic states of two $\tau$ leptons and two jets in proton-proton collisions at $\sqrt{s}=13 \mathrm{TeV}$, J. High Energy Phys. 03 (2019) 170.

[69] CMS Collaboration, Search for a singly produced thirdgeneration scalar leptoquark decaying to a $\tau$ lepton and a bottom quark in proton-proton collisions at $\sqrt{s}=13 \mathrm{TeV}$, J. High Energy Phys. 07 (2018) 115.

[70] ATLAS Collaboration, The ATLAS experiment at the CERN large hadron collider, J. Instrum. 3, S08003 (2008).

[71] ATLAS Collaboration, ATLAS insertable B-layer: Technical design report, Report No. ATLAS-TDR-19; Report No. CERN-LHCC-2010-013, 2010, https://cds.cern.ch/ record/1291633; Addendum, Report No. ATLAS-TDR19-ADD-1; Report No. CERN-LHCC-2012-009, 2012, https://cds.cern.ch/record/1451888.

[72] B. Abbott et al., Production and integration of the ATLAS Insertable B-Layer, J. Instrum. 13, T05008 (2018).

[73] ATLAS Collaboration, Operation of the ATLAS trigger system in Run 2, J. Instrum. 15, P10004 (2020).

[74] ATLAS Collaboration, The ATLAS Collaboration software and firmware, Report No. ATL-SOFT-PUB-2021001, 2021, https://cds.cern.ch/record/2767187.

[75] ATLAS Collaboration, Performance of the missing transverse momentum triggers for the ATLAS detector during Run-2 data taking, J. High Energy Phys. 08 (2020) 080.

[76] ATLAS Collaboration, ATLAS data quality operations and performance for 2015-2018 data-taking, J. Instrum. 15, P04003 (2020).

[77] ATLAS Collaboration, Luminosity determination in $p p$ collisions at $\sqrt{s}=13 \mathrm{TeV}$ using the ATLAS detector at the LHC, Report No. ATLAS-CONF-2019-021, 2019, https://cds.cern.ch/record/2677054.

[78] G. Avoni et al., The new LUCID-2 detector for luminosity measurement and monitoring in ATLAS, J. Instrum. 13, P07017 (2018).

[79] ATLAS Collaboration, The ATLAS simulation infrastructure, Eur. Phys. J. C 70, 823 (2010).

[80] S. Agostinelli et al. (GEANT4 Collaboration), Geant4-a simulation toolkit, Nucl. Instrum. Methods Phys. Res., Sect. A 506, 250 (2003).

[81] ATLAS Collaboration, The simulation principle and performance of the ATLAS fast calorimeter simulation FastCaloSim, Report No. ATL-PHYS-PUB-2010-013, 2010, https://cds.cern.ch/record/1300517.

[82] E. Bothmann et al., Event generation with SHERPA 2.2, SciPost Phys. 7, 034 (2019).
[83] ATLAS Collaboration, ATLAS Pythia8 tunes to $7 \mathrm{TeV}$ data, Report No. ATL-PHYS-PUB-2014-021, 2014, https://cds.cern.ch/record/1966419.

[84] D. J. Lange, The EvtGen particle decay simulation package, Nucl. Instrum. Methods Phys. Res., Sect. A 462, 152 (2001).

[85] T. Sjöstrand, S. Mrenna, and P. Skands, A brief introduction to Pythia8.1, Comput. Phys. Commun. 178, 852 (2008).

[86] R. D. Ball et al., Parton distributions with LHC data, Nucl. Phys. B867, 244 (2013).

[87] ATLAS Collaboration, The PYTHIA8 A3 tune description of ATLAS minimum bias and inelastic measurements incorporating the Donnachie-Landshoff diffractive model, Report NO. ATL-PHYS-PUB-2016-017, 2016, https://cds .cern.ch/record/2206965.

[88] S. Frixione, P. Nason, and G. Ridolfi, A positive-weight next-to-leading-order Monte Carlo for heavy flavour hadroproduction, J. High Energy Phys. 09 (2007) 126.

[89] P. Nason, A new method for combining NLO QCD with shower Monte Carlo algorithms, J. High Energy Phys. 11 (2004) 040.

[90] S. Frixione, P. Nason, and C. Oleari, Matching NLO QCD computations with parton shower simulations: The PowHEG method, J. High Energy Phys. 11 (2007) 070.

[91] S. Alioli, P. Nason, C. Oleari, and E. Re, A general framework for implementing NLO calculations in shower Monte Carlo programs: The PowHEG Box , J. High Energy Phys. 06 (2010) 043.

[92] T. Sjöstrand, S. Ask, J. R. Christiansen, R. Corke, N. Desai, P. Ilten, S. Mrenna, S. Prestel, C. O. Rasmussen, and P. Z. Skands, An introduction to PYTHIA8.2, Comput. Phys. Commun. 191, 159 (2015).

[93] M. Czakon and A. Mitov, Top++: A program for the calculation of the top-pair cross-section at hadron colliders, Comput. Phys. Commun. 185, 2930 (2014).

[94] R. D. Ball et al., Parton distributions for the LHC run II, J. High Energy Phys. 04 (2015) 040.

[95] E. Re, Single-top Wt-channel production matched with parton showers using the PowHEG method, Eur. Phys. J. C 71, 1547 (2011).

[96] M. Aliev, H. Lacker, U. Langenfeld, S. Moch, P. Uwer, and M. Wiedermann, HATHOR-Hadronic Top and Heavy quarks cross section calculator, Comput. Phys. Commun. 182, 1034 (2011).

[97] P. Kant, O. M. Kind, T. Kintscher, T. Lohse, T. Martini, S. Mölbitz, P. Rieck, and P. Uwer, HatHor for single topquark production: Updated predictions and uncertainty estimates for single top-quark production in hadronic collisions, Comput. Phys. Commun. 191, 74 (2015).

[98] N. Kidonakis, Two-loop soft anomalous dimensions for single top quark associated production with a $W^{-}$or $H^{-}$, Phys. Rev. D 82, 054018 (2010).

[99] N. Kidonakis, Top quark production, Proceedings, Helmholtz International Summer School on Physics of Heavy Quarks and Hadrons (HQ 2013), (JINR, Dubna, Russia, 2013), p. 139.

[100] C. Anastasiou, L. J. Dixon, K. Melnikov, and F. Petriello, High precision QCD at hadron colliders: Electroweak gauge boson rapidity distributions at next-to-next-to leading order, Phys. Rev. D 69, 094008 (2004). 
[101] T. Gleisberg and S. Höche, Comix, a new matrix element generator, J. High Energy Phys. 12 (2008) 039.

[102] S. Schumann and F. Krauss, A parton shower algorithm based on Catani-Seymour dipole factorisation, J. High Energy Phys. 03 (2008) 038.

[103] ATLAS Collaboration, Multi-boson simulation for $13 \mathrm{TeV}$ ATLAS analyses, Report No. ATL-PHYS-PUB-2017-005, 2017, https://cds.cern.ch/record/2261933.

[104] J. Alwall, R. Frederix, S. Frixione, V. Hirschi, F. Maltoni, O. Mattelaer, H.-S. Shao, T. Stelzer, P. Torrielli, and M. Zaro, The automated computation of tree-level and nextto-leading order differential cross sections, and their matching to parton shower simulations, J. High Energy Phys. 07 (2014) 079.

[105] ATLAS Collaboration, Modelling of the $t \bar{t} H$ and $t \bar{t} V(V=$ $W, Z$ ) processes for $\sqrt{s}=13 \mathrm{TeV}$ ATLAS analyses, Report No. ATL-PHYS-PUB-2016-005, 2016, https://cds .cern.ch/record/2120826.

[106] H. B. Hartanto, B. Jäger, L. Reina, and D. Wackeroth, Higgs boson production in association with top quarks in the Powheg Box, Phys. Rev. D 91, 094003 (2015).

[107] W. Beenakker, C. Borschensky, M. Krämer, A. Kulesza, and E. Laenen, NNLL-fast: Predictions for coloured supersymmetric particle production at the LHC with threshold and Coulomb resummation, J. High Energy Phys. 12 (2016) 133.

[108] W. Beenakker, M. Krämer, T. Plehn, M. Spira, and P. Zerwas, Stop production at hadron colliders, Nucl. Phys. B515, 3 (1998).

[109] W. Beenakker, S. Brensing, M. Krämer, A. Kulesza, E. Laenen, and I. Niessen, Supersymmetric top and bottom squark production at hadron colliders, J. High Energy Phys. 08 (2010) 098.

[110] W. Beenakker, C. Borschensky, R. Heger, M. Krämer, A. Kulesza, and E. Laenen, NNLL resummation for stop pairproduction at the LHC, J. High Energy Phys. 05 (2016) 153.

[111] P. Artoisenet, R. Frederix, O. Mattelaer, and R. Rietkerk, Automatic spin-entangled decays of heavy resonances in Monte Carlo simulations, J. High Energy Phys. 03 (2013) 015.

[112] L. Lönnblad, Correcting the colour-dipole cascade model with fixed order matrix elements, J. High Energy Phys. 05 (2002) 046.

[113] L. Lönnblad and S. Prestel, Matching tree-level matrix elements with interleaved showers, J. High Energy Phys. 03 (2012) 019.

[114] T. Mandal, S. Mitra, and S. Seth, Pair production of scalar leptoquarks at the LHC to NLO parton shower accuracy, Phys. Rev. D 93, 035018 (2016).

[115] M. Krämer, T. Plehn, M. Spira, and P. Zerwas, Pair production of scalar leptoquarks at the CERN LHC, Phys. Rev. D 71, 057503 (2005).

[116] M. Krämer, T. Plehn, M. Spira, and P. Zerwas, Pair Production of Scalar Leptoquarks at the Fermilab Tevatron, Phys. Rev. Lett. 79, 341 (1997).

[117] A. Belyaev, C. Leroy, R. Mehdiyev, and A. Pukhov, Leptoquark single and pair production at LHC with CalcHEP/CompHEP in the complete model, J. High Energy Phys. 09 (2005) 005.
[118] C. Borschensky, B. Fuks, A. Kulesza, and D. Schwartländer, Scalar leptoquark pair production at hadron colliders, Phys. Rev. D 101, 115017 (2020).

[119] M. J. Baker, J. Fuentes-Martín, G. Isidori, and M. König, High- $p_{T}$ signatures in vector-leptoquark models, Eur. Phys. J. C 79, 334 (2019).

[120] J. Blümlein, E. Boos, and A. Kryukov, Leptoquark pair production in hadronic interactions, Z. Phys. C 76, 137 (1997).

[121] ATLAS Collaboration, Vertex Reconstruction Performance of the ATLAS Detector at $\sqrt{s}=13 \mathrm{TeV}$, Report No. ATL-PHYS-PUB-2015-026, 2015, https://cds.cern.ch/ record/2037717.

[122] ATLAS Collaboration, Selection of jets produced in $13 \mathrm{TeV}$ proton-proton collisions with the ATLAS detector, Report No. ATLAS-CONF-2015-029, 2015, https://cds .cern.ch/record/2037702.

[123] ATLAS Collaboration, Jet reconstruction and performance using particle flow with the ATLAS detector, Eur. Phys. J. C 77, 466 (2017).

[124] M. Cacciari, G. P. Salam, and G. Soyez, The anti- $k_{t}$ jet clustering algorithm, J. High Energy Phys. 04 (2008) 063.

[125] M. Cacciari, G. P. Salam, and G. Soyez, FastJet user manual, Eur. Phys. J. C 72, 1896 (2012).

[126] ATLAS Collaboration, Jet energy scale and resolution measured in proton-proton collisions at $\sqrt{s}=13 \mathrm{TeV}$ with the ATLAS detector, Eur. Phys. J. C 81, 689 (2021).

[127] ATLAS Collaboration, Tagging and suppression of pileup jets with the ATLAS detector, Report No. ATLASCONF2014-018, 2014, https://cds.cern.ch/record/1700870.

[128] ATLAS Collaboration, ATLAS $b$-jet identification performance and efficiency measurement with $t \bar{t}$ events in $p p$ collisions at $\sqrt{s}=13 \mathrm{TeV}$, Eur. Phys. J. C 79, 970 (2019).

[129] ATLAS Collaboration, Optimisation and performance studies of the ATLAS $b$-tagging algorithms for the 2017-18 LHC run, Report No. ATL-PHYS-PUB-2017013, 2017, https://cds.cern.ch/record/2273281.

[130] ATLAS Collaboration, Topological cell clustering in the ATLAS calorimeters and its performance in LHC Run 1, Eur. Phys. J. C 77, 490 (2017).

[131] ATLAS Collaboration, Identification and energy calibration of hadronically decaying tau leptons with the ATLAS experiment in $p p$ collisions at $\sqrt{s}=8 \mathrm{TeV}$, Eur. Phys. J. C 75, 303 (2015).

[132] ATLAS Collaboration, Measurement of the tau lepton reconstruction and identification performance in the ATLAS experiment using $p p$ collisions at $\sqrt{s}=13 \mathrm{TeV}$, Report No. ATLAS-CONF-2017-029, 2017, https://cds .cern.ch/record/2261772.

[133] ATLAS Collaboration, Reconstruction of hadronic decay products of tau leptons with the ATLAS experiment, Eur. Phys. J. C 76, 295 (2016).

[134] ATLAS Collaboration, Identification of hadronic tau lepton decays using neural networks in the ATLAS experiment, Report No. ATL-PHYS-PUB-2019-033, 2019, https://cds.cern.ch/record/2688062.

[135] ATLAS Collaboration, Electron reconstruction and identification in the ATLAS experiment using the 2015 and 2016 LHC proton-proton collision data at $\sqrt{s}=13 \mathrm{TeV}$, Eur. Phys. J. C 79, 639 (2019). 
[136] ATLAS Collaboration, Electron and photon performance measurements with the ATLAS detector using the 20152017 LHC proton-proton collision data, J. Instrum. 14, P12006 (2019).

[137] ATLAS Collaboration, Muon reconstruction and identification efficiency in ATLAS using the full Run $2 p p$ collision data set at $\sqrt{s}=13 \mathrm{TeV}$, Eur. Phys. J. C 81, 578 (2021).

[138] ATLAS Collaboration, Performance of missing transverse momentum reconstruction with the ATLAS detector using proton-proton collisions at $\sqrt{s}=13 \mathrm{TeV}$, Eur. Phys. J. C 78, 903 (2018).

[139] ATLAS Collaboration, $E_{\mathrm{T}}^{\text {miss }}$ performance in the ATLAS detector using 2015-2016 LHC $p p$ collisions, Report No. ATLAS-CONF-2018-023, 2018, https://cds.cern.ch/ record/2625233.

[140] G. Cowan, K. Cranmer, E. Gross, and O. Vitells, Asymptotic formulae for likelihood-based tests of new physics, Eur. Phys. J. C 71, 1554 (2011); 73, 2501(E) (2013).

[141] C. G. Lester and D. J. Summers, Measuring masses of semi-invisibly decaying particles pair produced at hadron colliders, Phys. Lett. B 463, 99 (1999).

[142] C. G. Lester and B. Nachman, Bisection-based asymmetric MT2 computation: A higher precision calculator than existing symmetric methods, J. High Energy Phys. 03 (2015) 100.

[143] M. Baak, G. J. Besjes, D. Côté, A. Koutsman, J. Lorenz, and D. Short, HistFitter software framework for statistical data analysis, Eur. Phys. J. C 75, 153 (2015).

[144] J. Butterworth et al., PDF4LHC recommendations for LHC Run II, J. Phys. G 43, 023001 (2016).
[145] S. Frixione, E. Laenen, P. Motylinski, C. White, and B. R. Webber, Single-top hadroproduction in association with a W boson, J. High Energy Phys. 07 (2008) 029.

[146] S. Catani, F. Krauss, R. Kuhn, and B. R. Webber, QCD matrix elements + parton showers, J. High Energy Phys. 11 (2001) 063.

[147] S. Höche, F. Krauss, S. Schumann, and F. Siegert, QCD matrix elements and truncated showers, J. High Energy Phys. 05 (2009) 053.

[148] ATLAS Collaboration, Probing the Quantum Interference between Singly and Doubly Resonant Top-Quark Production in $p p$ Collisions at $\sqrt{s}=13 \mathrm{TeV}$ with the ATLAS Detector, Phys. Rev. Lett. 121, 152002 (2018).

[149] ATLAS Collaboration, Studies on top-quark Monte Carlo modelling for Top2016, Report No. ATL-PHYSPUB2016-020, 2016, https://cds.cern.ch/record/2216168.

[150] A. L. Read, Presentation of search results: The $C L_{S}$ technique, J. Phys. G 28, 2693 (2002).

[151] R. D. Cousins, J. T. Linnemann, and J. Tucker, Evaluation of three methods for calculating statistical significance when incorporating a systematic uncertainty into a test of the background-only hypothesis for a Poisson process, Nucl. Instrum. Methods Phys. Res., Sect. A 595, 480 (2008).

[152] The LEP2 SUSY Working Group and ALEPH, DELPHI, L3, OPAL experiments, Combined LEP Selectron/Smuon/ Stau results, 183-208 GeV, http://lepsusy.web.cern.ch/ lepsusy/www/sleptons_summer04/slep_final.html, 2004.

[153] ATLAS Collaboration, ATLAS computing acknowledgements, Report No. ATL-SOFT-PUB-2021-003, https://cds .cern.ch/record/2776662.

G. Aad,${ }^{99}$ B. Abbott, ${ }^{125}$ D. C. Abbott, ${ }^{100}$ A. Abed Abud, ${ }^{34}$ K. Abeling, ${ }^{51}$ D. K. Abhayasinghe, ${ }^{91}$ S. H. Abidi, ${ }^{27}$ O. S. AbouZeid ${ }^{38}$ H. Abramowicz, ${ }^{158}$ H. Abreu, ${ }^{157}$ Y. Abulaiti, ${ }^{5}$ A. C. Abusleme Hoffman, ${ }^{143 a}$ B. S. Acharya, ${ }^{64 a, 64 b, b}$ B. Achkar, ${ }^{51}$ L. Adam, ${ }^{97}$ C. Adam Bourdarios, ${ }^{4}$ L. Adamczyk,${ }^{81 a}$ L. Adamek, ${ }^{163}$ J. Adelman, ${ }^{118}$ A. Adiguzel, ${ }^{11 c, c}$ S. Adorni,${ }^{52}$ T. Adye ${ }^{140}$ A. A. Affolder,${ }^{142}$ Y. Afik, ${ }^{157}$ C. Agapopoulou, ${ }^{62}$ M. N. Agaras, ${ }^{12}$ J. Agarwala, ${ }^{68,68 b}$ A. Aggarwal, ${ }^{116}$ C. Agheorghiesei, ${ }^{25 c}$ J. A. Aguilar-Saavedra, ${ }^{136 f, 136 a, d}$ A. Ahmad,${ }^{34}$ F. Ahmadov, ${ }^{77}$ W. S. Ahmed,${ }^{101}$ X. Ai ${ }^{44}$ G. Aielli, ${ }^{71 a, 71 b}$ S. Akatsuka, ${ }^{83}$ M. Akbiyik, ${ }^{97}$ T. P. A. Åkesson, ${ }^{94}$ A. V. Akimov, ${ }^{108}$ K. Al Khoury, ${ }^{37}$ G. L. Alberghi, ${ }^{21 b, 21 a}$ J. Albert, ${ }^{172}$ M. J. Alconada Verzini,${ }^{86}$ S. Alderweireldt, ${ }^{48}$ M. Aleksa,${ }^{34}$ I. N. Aleksandrov, ${ }^{77}$ C. Alexa, ${ }^{25 b}$ T. Alexopoulos, ${ }^{9}$ A. Alfonsi, ${ }^{117}$ F. Alfonsi, ${ }^{21 b, 21 a}$ M. Alhroob, ${ }^{125}$ B. Ali, ${ }^{138}$ S. Ali, ${ }^{155}$ M. Aliev, ${ }^{162}$ G. Alimonti,${ }^{66}$ C. Allaire, ${ }^{34}$ B. M. M. Allbrooke, ${ }^{153}$ P. P. Allport, ${ }^{19}$ A. Aloisio, ${ }^{67 a, 67 b}$ F. Alonso, ${ }^{86}$ C. Alpigiani, ${ }^{145}$ E. Alunno Camelia, ${ }^{71 a, 71 b}$ M. Alvarez Estevez, ${ }^{96}$ M. G. Alviggi, ${ }^{67 a, 67 b}$ Y. Amaral Coutinho, ${ }^{78 b}$ A. Ambler, ${ }^{101}$ L. Ambroz, ${ }^{131}$ C. Amelung, ${ }^{34}$ D. Amidei, ${ }^{103}$ S. P. Amor Dos Santos, ${ }^{136 a}$ S. Amoroso, ${ }^{44}$ C. S. Amrouche,${ }^{52}$ C. Anastopoulos, ${ }^{146}$ N. Andari, ${ }^{141}$ T. Andeen, ${ }^{10}$ J. K. Anders ${ }^{18}$ S. Y. Andrean, ${ }^{43 a, 43 b}$ A. Andreazza, ${ }^{66 a, 66 b}$ V. Andrei, ${ }^{59 a}$ S. Angelidakis, ${ }^{8}$ A. Angerami, ${ }^{37}$ A. V. Anisenkov, ${ }^{119 b, 119 a}$ A. Annovi, ${ }^{69 a}$ C. Antel, ${ }^{52}$ M. T. Anthony, ${ }^{146}$ E. Antipov, ${ }^{126}$ M. Antonelli, ${ }^{49}$ D. J. A. Antrim, ${ }^{16}$ F. Anulli, ${ }^{70 a}$ M. Aoki,${ }^{79}$ J. A. Aparisi Pozo, ${ }^{170}$ M. A. Aparo, ${ }^{153}$ L. Aperio Bella ${ }^{44}$ N. Aranzabal, ${ }^{34}$ V. Araujo Ferraz, ${ }^{78 a}$ C. Arcangeletti, ${ }^{49}$ A. T. H. Arce, ${ }^{47}$ E. Arena,${ }^{88}$ J-F. Arguin, ${ }^{107}$ S. Argyropoulos,${ }^{50}$ J.-H. Arling, ${ }^{44}$ A. J. Armbruster, ${ }^{34}$ A. Armstrong, ${ }^{167}$ O. Arnaez, ${ }^{163}$ H. Arnold,${ }^{34}$ Z. P. Arrubarrena Tame, ${ }^{111}$ G. Artoni, ${ }^{131}$ H. Asada, ${ }^{114}$ K. Asai, ${ }^{123}$ S. Asai, ${ }^{160}$ N. A. Asbah, ${ }^{57}$ E. M. Asimakopoulou, ${ }^{168}$ L. Asquith, ${ }^{153}$ J. Assahsah, ${ }^{33 \mathrm{~d}}$ K. Assamagan, ${ }^{27}$ R. Astalos, ${ }^{26 a}$ R. J. Atkin, ${ }^{31 a}$ M. Atkinson, ${ }^{169}$ N. B. Atlay, ${ }^{17}$ H. Atmani, ${ }^{58 b}$ P. A. Atmasiddha, ${ }^{103}$ K. Augsten, ${ }^{138}$ S. Auricchio, ${ }^{67 a, 67 b}$ V. A. Austrup, ${ }^{178}$ G. Avolio, ${ }^{34}$ M. K. Ayoub, ${ }^{13 \mathrm{c}}$ G. Azuelos, ${ }^{107, \mathrm{e}}$ D. Babal, ${ }^{26 a}$ H. Bachacou, ${ }^{141}$ K. Bachas, ${ }^{159}$ F. Backman, ${ }^{43 a, 43 b}$ A. Badea ${ }^{57}$ P. Bagnaia, ${ }^{70 a, 70 b}$ H. Bahrasemani, ${ }^{149}$ A. J. Bailey, ${ }^{170}$ V. R. Bailey, ${ }^{169}$ J. T. Baines,${ }^{140}$ C. Bakalis, ${ }^{9}$ O. K. Baker, ${ }^{179}$ 
P. J. Bakker, ${ }^{117}$ E. Bakos, ${ }^{14}$ D. Bakshi Gupta, ${ }^{7}$ S. Balaji ${ }^{154}$ R. Balasubramanian, ${ }^{117}$ E. M. Baldin, ${ }^{19 b, 119 a}$ P. Balek, ${ }^{139}$ E. Ballabene ${ }^{66 a, 66 b}$ F. Balli, ${ }^{141}$ W. K. Balunas, ${ }^{131}$ J. Balz,${ }^{97}$ E. Banas ${ }^{82}$ M. Bandieramonte, ${ }^{135}$ A. Bandyopadhyay, ${ }^{17}$

L. Barak, ${ }^{158}$ E. L. Barberio, ${ }^{102}$ D. Barberis, ${ }^{53 b, 53 a}$ M. Barbero, ${ }^{99}$ G. Barbour, ${ }^{92}$ K. N. Barends, ${ }^{31 a}$ T. Barillari, ${ }^{112}$ M-S. Barisits, ${ }^{34}$ J. Barkeloo, ${ }^{128}$ T. Barklow, ${ }^{150}$ B. M. Barnett, ${ }^{140}$ R. M. Barnett, ${ }^{16}$ A. Baroncelli, ${ }^{58 a}$ G. Barone,${ }^{27}$ A. J. Barr, ${ }^{131}$ L. Barranco Navarro, ${ }^{43 a, 43 b}$ F. Barreiro, ${ }^{96}$ J. Barreiro Guimarães da Costa, ${ }^{13 a}$ U. Barron, ${ }^{158}$ S. Barsov, ${ }^{134}$ F. Bartels,${ }^{59 a}$ R. Bartoldus, ${ }^{150}$ G. Bartolini, ${ }^{99}$ A. E. Barton, ${ }^{87}$ P. Bartos ${ }^{26 a}$ A. Basalaev, ${ }^{44}$ A. Basan, ${ }^{97}$ I. Bashta, ${ }^{72 a, 72 b}$ A. Bassalat, ${ }^{62}$ M. J. Basso, ${ }^{163}$ C. R. Basson, ${ }^{98}$ R. L. Bates, ${ }^{55}$ S. Batlamous, ${ }^{33 \mathrm{e}}$ J. R. Batley, ${ }^{30}$ B. Batool,${ }^{148}$ M. Battaglia, ${ }^{142}$ M. Bauce, ${ }^{70 a}, 70 \mathrm{~b}$ F. Bauer, ${ }^{141, a}$ P. Bauer, ${ }^{22}$ H. S. Bawa, ${ }^{29}$ A. Bayirli, ${ }^{11 \mathrm{c}}$ J. B. Beacham, ${ }^{47}$ T. Beau, ${ }^{132}$ P. H. Beauchemin, ${ }^{166}$ F. Becherer, ${ }^{50}$ P. Bechtle, ${ }^{22}$ H. P. Beck, ${ }^{18, f}$ K. Becker, ${ }^{174}$ C. Becot,${ }^{44}$ A. J. Beddall, ${ }^{11 a}$ V. A. Bednyakov, ${ }^{77}$ C. P. Bee, ${ }^{152}$ T. A. Beermann, ${ }^{178}$ M. Begalli, ${ }^{78 b}$ M. Begel,${ }^{27}$ A. Behera, ${ }^{152}$ J. K. Behr, ${ }^{44}$ C. Beirao Da Cruz E Silva, ${ }^{34}$ J. F. Beirer, ${ }^{51,34}$ F. Beisiegel, ${ }^{22}$ M. Belfkir, ${ }^{4}$ G. Bella, ${ }^{158}$ L. Bellagamba, ${ }^{21 \mathrm{~b}}$ A. Bellerive, ${ }^{32}$ P. Bellos, ${ }^{19}$ K. Beloborodov, ${ }^{119 b, 119 \mathrm{a}}$ K. Belotskiy, ${ }^{109}$ N. L. Belyaev, ${ }^{109}$ D. Benchekroun, ${ }^{33 a}$ Y. Benhammou, ${ }^{158}$ D. P. Benjamin, ${ }^{5}$ M. Benoit, ${ }^{27}$ J. R. Bensinger, ${ }^{24}$ S. Bentvelsen, ${ }^{117}$ L. Beresford, ${ }^{131}$ M. Beretta, ${ }^{49}$ D. Berge,${ }^{17}$ E. Bergeaas Kuutmann, ${ }^{168}$ N. Berger, ${ }^{4}$ B. Bergmann, ${ }^{138}$ L. J. Bergsten, ${ }^{24}$ J. Beringer, ${ }^{16}$ S. Berlendis, ${ }^{6}$ G. Bernardi ${ }^{132}$ C. Bernius, ${ }^{150}$ F. U. Bernlochner, ${ }^{22}$ T. Berry, ${ }^{91}$ P. Berta, ${ }^{44}$ A. Berthold, ${ }^{46}$ I. A. Bertram, ${ }^{87}$ O. Bessidskaia Bylund, ${ }^{178}$ S. Bethke, ${ }^{112}$ A. Betti, ${ }^{40}$ A. J. Bevan, ${ }^{90}$ S. Bhatta, ${ }^{152}$ D. S. Bhattacharya, ${ }^{173}$ P. Bhattarai, ${ }^{24}$ V. S. Bhopatkar, ${ }^{5}$ R. Bi,${ }^{135}$ R. M. Bianchi, ${ }^{135}$ O. Biebel,${ }^{111}$ R. Bielski, ${ }^{34}$ N. V. Biesuz, ${ }^{69 a, 69 b}$ M. Biglietti, ${ }^{72 a}$

T. R. V. Billoud, ${ }^{138}$ M. Bindi, ${ }^{51}$ A. Bingul, ${ }^{11 \mathrm{~d}}$ C. Bini, ${ }^{70 a, 70 b}$ S. Biondi, ${ }^{21 b, 21 \mathrm{a}}$ C. J. Birch-sykes, ${ }^{98}$ G. A. Bird, ${ }^{19,141}$ M. Birman, ${ }^{176}$ T. Bisanz, ${ }^{34}$ J. P. Biswal, ${ }^{2}$ D. Biswas, ${ }^{177, g}$ A. Bitadze, ${ }^{98}$ C. Bittrich, ${ }^{46}$ K. Bjørke, ${ }^{130}$ I. Bloch, ${ }^{44}$ C. Blocker,${ }^{24}$ A. Blue ${ }^{55}$ U. Blumenschein, ${ }^{90}$ J. Blumenthal, ${ }^{97}$ G. J. Bobbink, ${ }^{117}$ V. S. Bobrovnikov, ${ }^{119 b, 119 a}$ D. Bogavac, ${ }^{12}$ A. G. Bogdanchikov, ${ }^{119 b, 119 a}$ C. Bohm, ${ }^{43 a}$ V. Boisvert, ${ }^{91}$ P. Bokan, ${ }^{44}$ T. Bold,${ }^{81 a}$ M. Bomben, ${ }^{132}$ M. Bona, ${ }^{90}$ M. Boonekamp, ${ }^{141}$ C. D. Booth, ${ }^{91}$ A. G. Borbély, ${ }^{55}$ H. M. Borecka-Bielska, ${ }^{107}$ L. S. Borgna, ${ }^{92}$ G. Borissov, ${ }^{87}$ D. Bortoletto, ${ }^{131}$ D. Boscherini, ${ }^{21 b}$ M. Bosman, ${ }^{12}$ J. D. Bossio Sola, ${ }^{101}$ K. Bouaouda, ${ }^{33 a}$ J. Boudreau, ${ }^{135}$ E. V. Bouhova-Thacker, ${ }^{87}$ D. Boumediene, ${ }^{36}$ R. Bouquet, ${ }^{132}$ A. Boveia, ${ }^{124}$ J. Boyd, ${ }^{34}$ D. Boye, ${ }^{27}$ I. R. Boyko, ${ }^{77}$ A. J. Bozson, ${ }^{91}$ J. Bracinik, ${ }^{19}$ N. Brahimi, ${ }^{58 d, 58 c}$ G. Brandt, ${ }^{178}$ O. Brandt, ${ }^{30}$ F. Braren, ${ }^{44}$ B. Brau ${ }^{100}$ J. E. Brau, ${ }^{128}$ W. D. Breaden Madden, ${ }^{55}$ K. Brendlinger, ${ }^{44}$ R. Brener, ${ }^{176}$ L. Brenner,${ }^{34}$ R. Brenner, ${ }^{168}$ S. Bressler, ${ }^{178}$ B. Brickwedde, ${ }^{97}$ D. L. Briglin, ${ }^{19}$ D. Britton, ${ }^{55}$ D. Britzger, ${ }^{12}$ I. Brock, ${ }^{22}$ R. Brock, ${ }^{104}$ G. Brooijmans, ${ }^{37}$ W. K. Brooks, ${ }^{143 e}$ E. Brost,${ }^{27}$ P. A. Bruckman de Renstrom, ${ }^{82}$ B. Brüers, ${ }^{44} \mathrm{D}$. Bruncko, ${ }^{26 \mathrm{~b}}$ A. Bruni, ${ }^{21 \mathrm{~b}} \mathrm{G}$. Bruni, ${ }^{21 \mathrm{~b}} \mathrm{M}$. Bruschi, ${ }^{21 \mathrm{~b}} \mathrm{~N}$. Bruscino ${ }^{70 \mathrm{a}, 70 \mathrm{~b}}$ L. Bryngemark, ${ }^{150}$ T. Buanes, ${ }^{15}$ Q. Buat, ${ }^{152}$ P. Buchholz, ${ }^{148}$ A. G. Buckley,${ }^{55}$ I. A. Budagov, ${ }^{77}$ M. K. Bugge, ${ }^{130}$ O. Bulekov, ${ }^{109}$ B. A. Bullard, ${ }^{57}$ T. J. Burch, ${ }^{118}$ S. Burdin, ${ }^{88}$ C. D. Burgard, ${ }^{44}$ A. M. Burger, ${ }^{126}$ B. Burghgrave, ${ }^{7}$ J. T. P. Burr, ${ }^{44}$ C. D. Burton, ${ }^{10}$ J. C. Burzynski, ${ }^{100}$ V. Büscher, ${ }^{97}$ P. J. Bussey,${ }^{55}$ J. M. Butler, ${ }^{23}$ C. M. Buttar,${ }^{55}$ J. M. Butterworth, ${ }^{92}$ W. Buttinger, ${ }^{140}$ C. J. Buxo Vazquez, ${ }^{104}$ A. R. Buzykaev, ${ }^{19 b, 119 a}$ G. Cabras, ${ }^{21 b, 21 a}$ S. Cabrera Urbán, ${ }^{170}$ D. Caforio, ${ }^{54}$ H. Cai, ${ }^{135}$ V. M. M. Cairo, ${ }^{150}$ O. Cakir,${ }^{3 a}$ N. Calace,${ }^{34}$ P. Calafiura, ${ }^{16}$ G. Calderini, ${ }^{132}$ P. Calfayan, ${ }^{63}$ G. Callea,${ }^{55}$ L. P. Caloba, ${ }^{78 b}$ A. Caltabiano, ${ }^{71 a, 71 b}$ S. Calvente Lopez, ${ }^{96}$ D. Calvet, ${ }^{36}$ S. Calvet, ${ }^{36}$ T. P. Calvet,,${ }^{99}$ M. Calvetti, ${ }^{6 a, 69 b}$ R. Camacho Toro, ${ }^{132}$ S. Camarda, ${ }^{34}$ D. Camarero Munoz,${ }^{96}$ P. Camarri, ${ }^{71 a, 71 b}$ M. T. Camerlingo, ${ }^{72 a, 72 b}$ D. Cameron, ${ }^{130}$ C. Camincher, ${ }^{172}$ M. Campanelli, ${ }^{92}$ A. Camplani,${ }^{38}$ V. Canale,${ }^{67 a, 67 b}$ A. Canesse, ${ }^{101}$ M. Cano Bret, ${ }^{75}$ J. Cantero, ${ }^{126}$ Y. Cao, ${ }^{169}$

M. Capua, ${ }^{39 b, 39 a}$ R. Cardarelli, ${ }^{71 a}$ F. Cardillo, ${ }^{170}$ G. Carducci, ${ }^{39 b, 39 a}$ T. Carli, ${ }^{34}$ G. Carlino, ${ }^{67 a}$ B. T. Carlson, ${ }^{135}$ E. M. Carlson, ${ }^{172,164 a}$ L. Carminati, ${ }^{66 a, 66 b}$ M. Carnesale, ${ }^{70 a, 70 b}$ R. M. D. Carney, ${ }^{150}$ S. Caron, ${ }^{116}$ E. Carquin, ${ }^{143 e}$ S. Carrá, ${ }^{44}$ G. Carratta, ${ }^{21 \mathrm{~b}, 21 \mathrm{a}}$ J. W. S. Carter, ${ }^{163}$ T. M. Carter, ${ }^{48}$ D. Casadei, ${ }^{31 \mathrm{c}}$ M. P. Casado, ${ }^{12, \mathrm{~h}}$ A. F. Casha, ${ }^{163}$ E. G. Castiglia, ${ }^{179}$ F. L. Castillo, ${ }^{59 a}$ L. Castillo Garcia, ${ }^{12}$ V. Castillo Gimenez, ${ }^{170}$ N. F. Castro, ${ }^{136 a, 136 e}$ A. Catinaccio, ${ }^{34}$ J. R. Catmore, ${ }^{130}$ A. Cattai ${ }^{34}$ V. Cavaliere ${ }^{27}$ N. Cavalli, ${ }^{21 b, 21 a}$ V. Cavasinni, ${ }^{69 a, 69 b}$ E. Celebi, ${ }^{11 b}$ F. Celli, ${ }^{131}$ K. Cerny, ${ }^{127}$ A. S. Cerqueira, ${ }^{78 a}$ A. Cerri, ${ }^{153}$ L. Cerrito, ${ }^{71 a, 71 b}$ F. Cerutti, ${ }^{16}$ A. Cervelli, ${ }^{21 b, 21 a}$ S. A. Cetin, ${ }^{11 b}$ Z. Chadi, ${ }^{33 a}$ D. Chakraborty, ${ }^{118}$ M. Chala, ${ }^{136 f}$ J. Chan, ${ }^{177}$ W. S. Chan, ${ }^{117}$ W. Y. Chan, ${ }^{88}$ J. D. Chapman, ${ }^{30}$ B. Chargeishvili, ${ }^{156 \mathrm{~b}}$ D. G. Charlton, ${ }^{19}$ T. P. Charman, ${ }^{90}$ M. Chatterjee, ${ }^{18}$ C. C. Chau, ${ }^{32}$ S. Chekanov, ${ }^{5}$ S. V. Chekulaev, ${ }^{164 a}$ G. A. Chelkov, ${ }^{77, i}$ A. Chen, ${ }^{103}$ B. Chen, ${ }^{158}$ C. Chen, ${ }^{58 a}$ C. H. Chen, ${ }^{76}$ H. Chen, ${ }^{13 \mathrm{c}}$ H. Chen, ${ }^{27}$ J. Chen, ${ }^{58 \mathrm{a}}$ J. Chen, ${ }^{37}$ J. Chen, ${ }^{24}$ S. Chen, ${ }^{133}$ S. J. Chen, ${ }^{13 \mathrm{c}}$ X. Chen, ${ }^{13 \mathrm{~b}}$ Y. Chen, ${ }^{58 \mathrm{a}}$ Y-H. Chen, ${ }^{44}$ C. L. Cheng, ${ }^{177}$ H. C. Cheng, ${ }^{60 a}$ H. J. Cheng, ${ }^{13 a}$ A. Cheplakov, ${ }^{77}$ E. Cheremushkina, ${ }^{44}$

R. Cherkaoui El Moursli, ${ }^{33 e}$ E. Cheu, ${ }^{6}$ K. Cheung, ${ }^{61}$ L. Chevalier, ${ }^{141}$ V. Chiarella, ${ }^{49}$ G. Chiarelli, ${ }^{69}$ G. Chiodini, ${ }^{65 a}$ A. S. Chisholm, ${ }^{19}$ A. Chitan, ${ }^{25 b}$ I. Chiu, ${ }^{160}$ Y. H. Chiu, ${ }^{172}$ M. V. Chizhov, ${ }^{77, j}$ K. Choi, ${ }^{10}$ A. R. Chomont, ${ }^{70 a, 70 b}$ Y. Chou, ${ }^{100}$ Y. S. Chow, ${ }^{117}$ L. D. Christopher, ${ }^{31 \mathrm{f}}$ M. C. Chu ${ }^{60 \mathrm{a}}$ X. Chu, ${ }^{13 \mathrm{a}, 13 \mathrm{~d}}$ J. Chudoba, ${ }^{137}$ J. J. Chwastowski, ${ }^{82}$ D. Cieri, ${ }^{112}$ K. M. Ciesla, ${ }^{82}$ V. Cindro, ${ }^{89}$ I. A. Cioară,${ }^{25 b}$ A. Ciocio, ${ }^{16}$ F. Cirotto, ${ }^{67 a, 67 b}$ Z. H. Citron, ${ }^{176, k}$ M. Citterio, ${ }^{66 a}$ D. A. Ciubotaru, ${ }^{25 b}$ 
B. M. Ciungu, ${ }^{163}$ A. Clark, ${ }^{52}$ P. J. Clark, ${ }^{48}$ S. E. Clawson, ${ }^{98}$ C. Clement,${ }^{43 a, 43 b}$ L. Clissa, ${ }^{21 b, 21 a}$ Y. Coadou, ${ }^{99}$ M. Cobal,${ }^{64 a 64 c}$ A. Coccaro, ${ }^{53 b}$ J. Cochran, ${ }^{76}$ R. F. Coelho Barrue, ${ }^{136 a}$ R. Coelho Lopes De Sa, ${ }^{100}$ S. Coelli, ${ }^{66 a}$ H. Cohen, ${ }^{158}$ A. E. C. Coimbra, ${ }^{34}$ B. Cole,${ }^{37}$ J. Collot, ${ }^{56}$ P. Conde Muiño, ${ }^{136 a, 136 h}$ S. H. Connell, ${ }^{31 \mathrm{c}}$ I. A. Connelly, ${ }^{55}$ E. I. Conroy, ${ }^{131}$ F. Conventi, ${ }^{67,1}$ H. G. Cooke, ${ }^{19}$ A. M. Cooper-Sarkar, ${ }^{131}$ F. Cormier, ${ }^{171}$ L. D. Corpe,${ }^{34}$ M. Corradi $,{ }^{70 a}, 70 b$ E. E. Corrigan, ${ }^{94}$ F. Corriveau, ${ }^{101, \mathrm{~m}}$ M. J. Costa, ${ }^{170}$ F. Costanza, ${ }^{4}$ D. Costanzo, ${ }^{146}$ B. M. Cote, ${ }^{124}$ G. Cowan, ${ }^{91}$ J. W. Cowley, ${ }^{30}$ J. Crane, ${ }^{98}$

K. Cranmer, ${ }^{122}$ R. A. Creager, ${ }^{133}$ S. Crépé-Renaudin, ${ }^{56}$ F. Crescioli, ${ }^{132}$ M. Cristinziani, ${ }^{148}$ M. Cristoforetti, ${ }^{73 a, 73 b, n}$ V. Croft, ${ }^{166}$ G. Crosetti, ${ }^{39 b, 39 a}$ A. Cueto, ${ }^{4}$ T. Cuhadar Donszelmann, ${ }^{167}$ H. Cui, ${ }^{13 a, 13 d}$ A. R. Cukierman, ${ }^{150}$ W. R. Cunningham, ${ }^{55}$ S. Czekierda ${ }^{82}$ P. Czodrowski, ${ }^{34}$ M. M. Czurylo, ${ }^{59 b}$ M. J. Da Cunha Sargedas De Sousa, ${ }^{58 a}$ J. V. Da Fonseca Pinto, ${ }^{78 b}$ C. Da Via, ${ }^{98}$ W. Dabrowski, ${ }^{81 a}$ T. Dado, ${ }^{45}$ S. Dahbi,${ }^{31 f}$ T. Dai, ${ }^{103}$ C. Dallapiccola, ${ }^{100}$ M. Dam, ${ }^{38}$ G. D'amen, ${ }^{27}$ V. D'Amico, ${ }^{72 a, 72 b}$ J. Damp, ${ }^{97}$ J. R. Dandoy, ${ }^{133}$ M. F. Daneri, ${ }^{28}$ M. Danninger, ${ }^{149}$ V. Dao, ${ }^{34}$ G. Darbo, ${ }^{53 b}$ S. Darmora, ${ }^{5}$ A. Dattagupta, ${ }^{128}$ S. D'Auria, ${ }^{66 a, 66 \mathrm{~b}}$ C. David,${ }^{164 \mathrm{~b}}$ T. Davidek, ${ }^{139}$ D. R. Davis, ${ }^{47}$ B. Davis-Purcell, ${ }^{32}$ I. Dawson,${ }^{90}$ K. De ${ }^{7}$ R. De Asmundis, ${ }^{67 a}$ M. De Beurs,${ }^{117}$ S. De Castro, ${ }^{21 b, 21 a}$ N. De Groot,${ }^{116}$ P. de Jong, ${ }^{117}$ H. De la Torre, ${ }^{104}$ A. De Maria, ${ }^{13 \mathrm{c}}$ D. De Pedis, ${ }^{70 a}$ A. De Salvo,${ }^{70 a}$ U. De Sanctis, ${ }^{71 a, 71 b}$ M. De Santis, ${ }^{71 a, 71 b}$ A. De Santo, ${ }^{153}$ J. B. De Vivie De Regie, ${ }^{56}$ D. V. Dedovich, ${ }^{77}$ J. Degens, ${ }^{117}$ A. M. Deiana, ${ }^{40}$ J. Del Peso, ${ }^{96}$ Y. Delabat Diaz,${ }^{44}$ F. Deliot, ${ }^{141}$ C. M. Delitzsch,${ }^{6}$ M. Della Pietra,${ }^{67 a, 67 b}$ D. Della Volpe, ${ }^{52}$ A. Dell'Acqua, ${ }^{34}$ L. Dell'Asta, ${ }^{66 a, 66 b}$ M. Delmastro, ${ }^{4}$ P. A. Delsart, ${ }^{56}$ S. Demers, ${ }^{179}$ M. Demichev, ${ }^{77}$ S. P. Denisov, ${ }^{120}$ L. D'Eramo, ${ }^{118}$ D. Derendarz, ${ }^{82}$ J. E. Derkaoui, ${ }^{33 d}$ F. Derue,${ }^{132}$ P. Dervan,${ }^{88}$ K. Desch,${ }^{22}$ K. Dette, ${ }^{163}$ C. Deutsch,${ }^{22}$ P. O. Deviveiros ${ }^{34}$ F. A. Di Bello, ${ }^{70 a, 70 b}$ A. Di Ciaccio,${ }^{71 a, 71 b}$ L. Di Ciaccio, ${ }^{4}$ C. Di Donato,${ }^{67 a, 67 b}$ A. Di Girolamo, ${ }^{34}$ G. Di Gregorio, ${ }^{69 a, 69 b}$ A. Di Luca ${ }^{73 a, 73 b}$ B. Di Micco, ${ }^{72 a, 72 b}$ R. Di Nardo, ${ }^{72 a, 72 b}$ C. Diaconu, ${ }^{99}$ F. A. Dias, ${ }^{117}$ T. Dias Do Vale, ${ }^{136 a}$ M. A. Diaz, ${ }^{143 a}$ F. G. Diaz Capriles, ${ }^{22}$ J. Dickinson, ${ }^{16}$ M. Didenko, ${ }^{170}$ E. B. Diehl, ${ }^{103}$ J. Dietrich, ${ }^{17}$ S. Díez Cornell, ${ }^{44}$ C. Diez Pardos, ${ }^{148}$ A. Dimitrievska, ${ }^{16}$ W. Ding, ${ }^{13 b}$ J. Dingfelder, ${ }^{22}$ S. J. Dittmeier, ${ }^{59 b}$ F. Dittus, ${ }^{34}$ F. Djama, ${ }^{99}$ T. Djobava, ${ }^{156 b}$ J. I. Djuvsland, ${ }^{15}$ M. A. B. Do Vale, ${ }^{144}$ D. Dodsworth, ${ }^{24}$ C. Doglioni, ${ }^{94}$ J. Dolejsi ${ }^{139}$ Z. Dolezal, ${ }^{139}$ M. Donadelli, ${ }^{78 c}$ B. Dong, ${ }^{58 c}$ J. Donini, ${ }^{36}$ A. D'onofrio, ${ }^{13 c}$ M. D'Onofrio, ${ }^{88}$ J. Dopke, ${ }^{140}$ A. Doria,${ }^{67 a}$ M. T. Dova,${ }^{86}$ A. T. Doyle,${ }^{55}$ E. Drechsler, ${ }^{149}$ E. Dreyer, ${ }^{149}$ T. Dreyer, ${ }^{51}$ A. S. Drobac, ${ }^{166}$ D. Du, ${ }^{58 b}$ T. A. du Pree,${ }^{117}$ F. Dubinin, ${ }^{108}$ M. Dubovsky,${ }^{26 a}$ A. Dubreuil,${ }^{52}$ E. Duchovni, ${ }^{176}$ G. Duckeck, ${ }^{111}$ O. A. Ducu, ${ }^{34,25 b}$ D. Duda, ${ }^{12}$ A. Dudarev,${ }^{34}$ M. D'uffizi, ${ }^{98}$ L. Duflot, ${ }^{62}$ M. Dührssen, ${ }^{34}$ C. Dülsen, ${ }^{178}$ A. E. Dumitriu, ${ }^{25 b}$ M. Dunford ${ }^{59 a}$ S. Dungs, ${ }^{45}$ A. Duperrin,${ }^{99}$ H. Duran Yildiz, ${ }^{3 a}$ M. Düren, ${ }^{54}$ A. Durglishvilii, ${ }_{156 b}$ B. Dutta,${ }^{44}$ D. Duvnjak, ${ }^{1}$ G. I. Dyckes, ${ }^{133}$ M. Dyndal, ${ }^{81 a}$ S. Dysch, ${ }^{98}$ B. S. Dziedzic,${ }^{82}$ B. Eckerova, ${ }^{26 a}$ M. G. Eggleston, ${ }^{47}$

E. Egidio Purcino De Souza, ${ }^{78 b}$ L. F. Ehrke, ${ }^{52}$ T. Eifert, ${ }^{7}$ G. Eigen, ${ }^{15}$ K. Einsweiler, ${ }^{16}$ T. Ekelof, ${ }^{168}$ Y. El Ghazali, ${ }^{33 b}$ H. El Jarrari, ${ }^{33 e}$ A. El Moussaouy, ${ }^{33 a}$ V. Ellajosyula, ${ }^{168}$ M. Ellert, ${ }^{168}$ F. Ellinghaus, ${ }^{178}$ A. A. Elliot, ${ }^{90}$ N. Ellis, ${ }^{34}$ J. Elmsheuser, ${ }^{27}$ M. Elsing, ${ }^{34}$ D. Emeliyanov, ${ }^{140}$ A. Emerman, ${ }^{37}$ Y. Enari, ${ }^{160}$ J. Erdmann, ${ }^{45}$ A. Ereditato, ${ }^{18}$ P. A. Erland, ${ }^{82}$ M. Errenst, ${ }^{178}$ M. Escalier, ${ }^{62}$ C. Escobar, ${ }^{170}$ O. Estrada Pastor, ${ }^{170}$ E. Etzion, ${ }^{158}$ G. Evans, ${ }^{136 a}$ H. Evans, ${ }^{63}$ M. O. Evans, ${ }^{153}$ A. Ezhilov, ${ }^{134}$ F. Fabbri, ${ }^{55}$ L. Fabbri, ${ }^{21 b, 21 a}$ V. Fabiani, ${ }^{116}$ G. Facini, ${ }^{174}$ R. M. Fakhrutdinov, ${ }^{120}$ S. Falciano,${ }^{70 a}$ P. J. Falke, ${ }^{22}$ S. Falke, ${ }^{34}$ J. Faltova, ${ }^{139}$ Y. Fan, ${ }^{13 a}$ Y. Fang, ${ }^{13 a}$ Y. Fang, ${ }^{13 a}$ G. Fanourakis, ${ }^{42}$ M. Fanti, ${ }^{66 a, 66 b}$ M. Faraj, ${ }^{58 c}$ A. Farbin, ${ }^{7}$ A. Farilla ${ }^{72 a}$ E. M. Farina, ${ }^{68 a, 68 b}$ T. Farooque ${ }^{104}$ S. M. Farrington,${ }^{48}$ P. Farthouat ${ }^{34}$ F. Fassi, ${ }^{33 e}$ D. Fassouliotis ${ }^{8}$ M. Faucci Giannelli, ${ }^{71 a, 71 b}$ W. J. Fawcett, ${ }^{30}$ L. Fayard, ${ }^{62}$ O. L. Fedin, ${ }^{134,0}$ M. Feickert, ${ }^{169}$ L. Feligioni, ${ }^{99}$ A. Fell, ${ }^{146}$ C. Feng, ${ }^{58 b}$ M. Feng, ${ }^{13 b}$ M. J. Fenton, ${ }^{167}$ A. B. Fenyuk, ${ }^{120}$ S. W. Ferguson, ${ }^{41}$ J. Ferrando, ${ }^{44}$ A. Ferrari, ${ }^{168}$ P. Ferrari, ${ }^{117}$ R. Ferrari, ${ }^{68 a}$ D. Ferrere, ${ }^{52}$ C. Ferretti, ${ }^{103}$ F. Fiedler, ${ }^{97}$ A. Filipčič,${ }^{89}$ F. Filthaut, ${ }^{116}$ M. C. N. Fiolhais,${ }^{136 a, 136 c, p}$ L. Fiorini, ${ }^{170}$ F. Fischer, ${ }^{111}$ W. C. Fisher, ${ }^{104}$ T. Fitschen, ${ }^{19}$ I. Fleck, ${ }^{148}$ P. Fleischmann, ${ }^{103}$ T. Flick, ${ }^{178}$ B. M. Flierl, ${ }^{111}$ L. Flores, ${ }^{133}$ L. R. Flores Castillo, ${ }^{60 a}$ F. M. Follega ${ }^{73 a, 73 b}$ N. Fomin,${ }^{15}$ J. H. Foo, ${ }^{163}$ G. T. Forcolin, ${ }^{73 a, 73 b}$ B. C. Forland ${ }^{63}$ A. Formica, ${ }^{141}$ F. A. Förster, ${ }^{12}$ A. C. Forti, ${ }^{98}$ E. Fortin, ${ }^{99}$ M. G. Foti, ${ }^{131}$ D. Fournier, ${ }^{62}$ H. Fox,${ }^{87}$ P. Francavilla, ${ }^{69 a, 69 b}$ S. Francescato,${ }^{70 a, 70 b}$ M. Franchini, ${ }^{21 b, 21 a}$ S. Franchino, ${ }^{59 a}$ D. Francis, ${ }^{34}$ L. Franco, ${ }^{4}$ L. Franconi, ${ }^{18}$ M. Franklin, ${ }^{57}$ G. Frattari, ${ }^{70 a, 70 b}$ A. C. Freegard, ${ }^{90}$ P. M. Freeman, ${ }^{19}$ B. Freund, ${ }^{107}$ W. S. Freund ${ }^{78 b}$ E. M. Freundlich,${ }^{45}$ D. Froidevaux,${ }^{34}$ J. A. Frost, ${ }^{131}$ Y. Fu, ${ }^{58 a}$ M. Fujimoto, ${ }^{123}$ E. Fullana Torregrosa, ${ }^{170}$ T. Fusayasu, ${ }^{113}$ J. Fuster, ${ }^{170}$ A. Gabrielli, ${ }^{21 b, 21 a}$ A. Gabrielli, ${ }^{34}$ P. Gadow, ${ }^{44}$ G. Gagliardi, ${ }^{53 b, 53 a}$ L. G. Gagnon, ${ }^{16}$ G. E. Gallardo, ${ }^{131}$ E. J. Gallas, ${ }^{131}$ B. J. Gallop, ${ }^{140}$ R. Gamboa Goni, ${ }^{90}$ K. K. Gan, ${ }^{124}$ S. Ganguly, ${ }^{176}$ J. Gao, ${ }^{58 a}$ Y. Gao ${ }^{48}$ Y. S. Gao, ${ }^{29, q}$ F. M. Garay Walls,${ }^{143 a}$ C. García, ${ }^{170}$ J. E. García Navarro, ${ }^{170}$ J. A. García Pascual, ${ }^{13 a}$ M. Garcia-Sciveres, ${ }^{16}$ R. W. Gardner,${ }^{35}$ D. Garg, ${ }^{75}$ S. Gargiulo, ${ }^{50}$ C. A. Garner, ${ }^{163}$ V. Garonne, ${ }^{130}$ S. J. Gasiorowski, ${ }^{145}$ P. Gaspar, ${ }^{78 b}$ G. Gaudio, ${ }^{68 \mathrm{a}}$ P. Gauzzi, ${ }^{70 a, 70 b}$ I. L. Gavrilenko, ${ }^{108}$ A. Gavrilyuk, ${ }^{121}$ C. Gay, ${ }^{171}$ G. Gaycken, ${ }^{44}$ E. N. Gazis, ${ }^{9}$ A. A. Geanta ${ }^{25 b}$ C. M. Gee, ${ }^{142}$ C. N. P. Gee, ${ }^{140}$ J. Geisen, ${ }^{94}$ M. Geisen, ${ }^{97}$ C. Gemme,${ }^{53 b}$ M. H. Genest, ${ }^{56}$ S. Gentile, ${ }^{70 a, 70 b}$ S. George, ${ }^{91}$ T. Geralis, ${ }^{42}$ L. O. Gerlach, ${ }^{51}$ P. Gessinger-Befurt, ${ }^{97}$ 
M. Ghasemi Bostanabad, ${ }^{172}$ M. Ghneimat, ${ }^{148}$ A. Ghosh, ${ }^{167}$ A. Ghosh,${ }^{75}$ B. Giacobbe,${ }^{21 b}$ S. Giagu, ${ }^{70 a, 70 b}$ N. Giangiacomi, ${ }^{163}$ P. Giannetti, ${ }^{69 a}$ A. Giannini, ${ }^{67,67 b}$ S. M. Gibson, ${ }^{91}$ M. Gignac, ${ }^{142}$ D. T. Gil,${ }^{81 b}$ B. J. Gilbert, ${ }^{37}$ D. Gillberg, ${ }^{32}$ G. Gilles, ${ }^{178}$ N. E. K. Gillwald, ${ }^{44}$ D. M. Gingrich, ${ }^{2, \mathrm{e}}$ M. P. Giordani, ${ }^{64,64 \mathrm{c}}$ P. F. Giraud,${ }^{141}$ G. Giugliarelli, ${ }^{64 \mathrm{a}, 64 \mathrm{c}}$ D. Giugni, ${ }^{6 \mathrm{a}}$

F. Giuli, ${ }^{71,71 b}$ I. Gkialas, ${ }^{8, r}$ E. L. Gkougkousis, ${ }^{12}$ P. Gkountoumis, ${ }^{9}$ L. K. Gladilin, ${ }^{110}$ C. Glasman, ${ }^{96}$ G. R. Gledhill, ${ }^{128}$ M. Glisic, ${ }^{128}$ I. Gnesi, ${ }^{39 b, s}$ M. Goblirsch-Kolb, ${ }^{24}$ D. Godin, ${ }^{107}$ S. Goldfarb, ${ }^{102}$ T. Golling, ${ }^{52}$ D. Golubkov, ${ }^{120}$ J. P. Gombas, ${ }^{104}$ A. Gomes, ${ }^{136 a, 136 b}$ R. Goncalves Gama, ${ }^{51}$ R. Gonçalo, ${ }^{136 a, 136 c}$ G. Gonella, ${ }^{128}$ L. Gonella, ${ }^{19}$ A. Gongadze, ${ }^{77}$ F. Gonnella, ${ }^{19}$ J. L. Gonski, ${ }^{37}$ S. González de la Hoz, ${ }^{170}$ S. Gonzalez Fernandez, ${ }^{12}$ R. Gonzalez Lopez, ${ }^{88}$ C. Gonzalez Renteria, ${ }^{16}$ R. Gonzalez Suarez, ${ }^{168}$ S. Gonzalez-Sevilla, ${ }^{52}$ G. R. Gonzalvo Rodriguez, ${ }^{170}$ R. Y. González Andana, ${ }^{143 a}$ L. Goossens, ${ }^{34}$ N. A. Gorasia, ${ }^{19}$ P. A. Gorbounov,${ }^{121}$ H. A. Gordon, ${ }^{27}$ B. Gorini, ${ }^{34}$ E. Gorini, ${ }^{65 a, 65 b}$ A. Gorišek, ${ }^{89}$ A. T. Goshaw, ${ }^{47}$ M. I. Gostkin, ${ }^{77}$ C. A. Gottardo, ${ }^{116}$ M. Gouighri, ${ }^{33 b}$ V. Goumarre, ${ }^{44}$ A. G. Goussiou, ${ }^{145}$ N. Govender, ${ }^{31 c}$ C. Goy, ${ }^{4}$ I. Grabowska-Bold, ${ }^{81 a}$ K. Graham, ${ }^{32}$ E. Gramstad, ${ }^{130}$ S. Grancagnolo, ${ }^{17}$ M. Grandi, ${ }^{153}$ V. Gratchev, ${ }^{134}$ P. M. Gravila, ${ }^{25 f}$ F. G. Gravili, ${ }^{65 a, 65 b}$ H. M. Gray, ${ }^{16}$ C. Grefe, ${ }^{22}$ I. M. Gregor, ${ }^{44}$ P. Grenier, ${ }^{150}$ K. Grevtsov, ${ }^{44}$ C. Grieco, ${ }^{12}$ N. A. Grieser, ${ }^{125}$ A. A. Grillo, ${ }^{142}$ K. Grimm, ${ }^{29, t}$ S. Grinstein, ${ }^{12, \mathrm{u}}$ J.-F. Grivaz, ${ }^{62}$ S. Groh, ${ }^{97}$ E. Gross, ${ }^{176}$ J. Grosse-Knetter, ${ }^{51}$ Z. J. Grout, ${ }^{92}$ C. Grud ${ }^{103}$ A. Grummer, ${ }^{115}$ J. C. Grundy, ${ }^{131}$ L. Guan, ${ }^{103}$ W. Guan, ${ }^{177}$ C. Gubbels, ${ }^{171}$ J. Guenther, ${ }^{34}$

J. G. R. Guerrero Rojas, ${ }^{170}$ F. Guescini, ${ }^{12}$ D. Guest, ${ }^{17}$ R. Gugel, ${ }^{97}$ A. Guida, ${ }^{44}$ T. Guillemin, ${ }^{4}$ S. Guindon, ${ }^{34}$ J. Guo, ${ }^{58 c}$ L. Guo,${ }^{62}$ Y. Guo, ${ }^{103}$ R. Gupta, ${ }^{44}$ S. Gurbuz, ${ }^{22}$ G. Gustavino, ${ }^{125}$ M. Guth,${ }^{50}$ P. Gutierrez, ${ }^{125}$ L. F. Gutierrez Zagazeta, ${ }^{133}$ C. Gutschow, ${ }^{92}$ C. Guyot, ${ }^{141}$ C. Gwenlan, ${ }^{131}$ C. B. Gwilliam, ${ }^{88}$ E. S. Haaland, ${ }^{130}$ A. Haas, ${ }^{122}$ M. Habedank, ${ }^{17}$ C. Haber, ${ }^{16}$ H. K. Hadavand, ${ }^{7}$ A. Hadef,${ }^{97}$ M. Haleem, ${ }^{173}$ J. Haley, ${ }^{126}$ J. J. Hall, ${ }^{146}$ G. Halladjian, ${ }^{104}$ G. D. Hallewell, ${ }^{99}$ L. Halser, ${ }^{18}$ K. Hamano, ${ }^{172}$ H. Hamdaoui, ${ }^{33 e}$ M. Hamer, ${ }^{22}$ G. N. Hamity, ${ }^{48}$ K. Han ${ }^{58 a}$ L. Han, ${ }^{13 \mathrm{c}}$ L. Han, ${ }^{58 a}$ S. Han, ${ }^{16}$ Y. F. Han, ${ }^{163}$ K. Hanagaki, ${ }^{79, v}$ M. Hance, ${ }^{142}$ M. D. Hank,${ }^{35}$ R. Hankache, ${ }^{98}$ E. Hansen, ${ }^{94}$ J. B. Hansen, ${ }^{38}$ J. D. Hansen, ${ }^{38}$ M. C. Hansen, ${ }^{22}$ P. H. Hansen, ${ }^{38}$ K. Hara, ${ }^{165}$ T. Harenberg, ${ }^{178}$ S. Harkusha, ${ }^{105}$ Y. T. Harris, ${ }^{131}$ P. F. Harrison, ${ }^{174}$ N. M. Hartman, ${ }^{150}$ N. M. Hartmann, ${ }^{111}$ Y. Hasegawa, ${ }^{147}$ A. Hasib, ${ }^{48}$ S. Hassani,${ }^{141}$ S. Haug,${ }^{18}$ R. Hauser, ${ }^{104}$ M. Havranek, ${ }^{138}$ C. M. Hawkes, ${ }^{19}$ R. J. Hawkings, ${ }^{34}$ S. Hayashida,${ }^{114}$ D. Hayden, ${ }^{104}$ C. Hayes, ${ }^{103}$ R. L. Hayes, ${ }^{171}$ C. P. Hays, ${ }^{131}$ J. M. Hays, ${ }^{90}$ H. S. Hayward ${ }^{88}$ S. J. Haywood, ${ }^{140}$ F. He, ${ }^{58 a}$ Y. He, ${ }^{161}$ Y. He,${ }^{132}$ M. P. Heath, ${ }^{48}$ V. Hedberg, ${ }^{94}$ A. L. Heggelund, ${ }^{130}$ N. D. Hehir, ${ }^{90}$

C. Heidegger, ${ }^{50}$ K. K. Heidegger, ${ }^{50}$ W. D. Heidorn, ${ }^{76}$ J. Heilman, ${ }^{32}$ S. Heim,,${ }^{44}$ T. Heim, ${ }^{16}$ B. Heinemann, ${ }^{44, w}$ J. G. Heinlein, ${ }^{133}$ J. J. Heinrich, ${ }^{128}$ L. Heinrich, ${ }^{34}$ J. Hejbal, ${ }^{137}$ L. Helary, ${ }^{44}$ A. Held, ${ }^{122}$ S. Hellesund, ${ }^{130}$ C. M. Helling, ${ }^{142}$ S. Hellman, ${ }^{43 a, 43 b}$ C. Helsens, ${ }^{34}$ R. C. W. Henderson, ${ }^{87}$ L. Henkelmann, ${ }^{30}$ A. M. Henriques Correia, ${ }^{34}$ H. Herde,${ }^{150}$ Y. Hernández Jiménez, ${ }^{152}$ H. Herr, ${ }^{97}$ M. G. Herrmann, ${ }^{111}$ T. Herrmann, ${ }^{46}$ G. Herten, ${ }^{50}$ R. Hertenberger ${ }^{111}$ L. Hervas, ${ }^{34}$ N. P. Hessey, ${ }^{164 a}$ H. Hibi, ${ }^{80}$ S. Higashino, ${ }^{79}$ E. Higón-Rodriguez, ${ }^{170}$ K. K. Hill, ${ }^{27}$ K. H. Hiller, ${ }^{44}$ S. J. Hillier, ${ }^{19}$ M. Hils, ${ }^{46}$ I. Hinchliffe, ${ }^{16}$ F. Hinterkeuser, ${ }^{22}$ M. Hirose, ${ }^{129}$ S. Hirose, ${ }^{165}$ D. Hirschbuehl, ${ }^{178}$ B. Hiti, ${ }^{89}$ O. Hladik, ${ }^{137}$ J. Hobbs, ${ }^{152}$ R. Hobincu, ${ }^{25 e}$ N. Hod, ${ }^{176}$ M. C. Hodgkinson, ${ }^{146}$ B. H. Hodkinson, ${ }^{30}$ A. Hoecker, ${ }^{34}$ J. Hofer, ${ }^{44}$ D. Hohn, ${ }^{50}$ T. Holm, ${ }^{22}$ T. R. Holmes, ${ }^{35}$ M. Holzbock, ${ }^{112}$ L. B. A. H. Hommels, ${ }^{30}$ B. P. Honan, ${ }^{98}$ T. M. Hong, ${ }^{135}$ J. C. Honig, ${ }^{50}$ A. Hönle, ${ }^{112}$

B. H. Hooberman, ${ }^{169}$ W. H. Hopkins, ${ }^{5}$ Y. Horii, ${ }^{114}$ P. Horn, ${ }^{46}$ L. A. Horyn, ${ }^{35}$ S. Hou, ${ }^{155}$ J. Howarth, ${ }^{55}$ J. Hoya, ${ }^{86}$ M. Hrabovsky, ${ }^{127}$ A. Hrynevich, ${ }^{106}$ T. Hryn'ova, ${ }^{4}$ P. J. Hsu,${ }^{61}$ S.-C. Hsu, ${ }^{145}$ Q. Hu, ${ }^{37}$ S. Hu, ${ }^{58 c}$ Y. F. Hu, ${ }^{13 a, 13 d, x}$ D. P. Huang,${ }^{92}$ X. Huang, ${ }^{13 c}$ Y. Huang, ${ }^{58 a}$ Y. Huang, ${ }^{13 a}$ Z. Hubacek, ${ }^{138}$ F. Hubaut, ${ }^{99}$ M. Huebner, ${ }^{22}$ F. Huegging, ${ }^{22}$ T. B. Huffman, ${ }^{131}$ M. Huhtinen, ${ }^{34}$ R. Hulsken, ${ }^{56}$ N. Huseynov, ${ }^{77, y}$ J. Huston, ${ }^{104}$ J. Huth, ${ }^{57}$ R. Hyneman, ${ }^{150}$ S. Hyrych, ${ }^{26 a}$ G. Iacobucci, ${ }^{52}$ G. Iakovidis, ${ }^{27}$ I. Ibragimov, ${ }^{148}$ L. Iconomidou-Fayard, ${ }^{62}$ P. Iengo, ${ }^{34}$ R. Ignazzi, ${ }^{38}$ R. Iguchi, ${ }^{160}$ T. Iizawa, ${ }^{52}$ Y. Ikegami, ${ }^{79}$

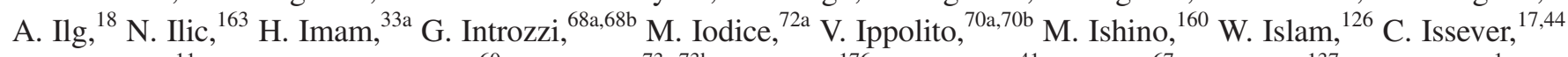
S. Istin, ${ }^{11 c, z}$ J. M. Iturbe Ponce, ${ }^{60 a}$ R. Iuppa, ${ }^{73 a, 73 b}$ A. Ivina, ${ }^{176}$ J. M. Izen, ${ }^{41}$ V. Izzo, ${ }^{67 a}$ P. Jacka, ${ }^{137}$ P. Jackson, ${ }^{1}$ R. M. Jacobs ${ }^{44}$ B. P. Jaeger, ${ }^{149}$ C. S. Jagfeld,${ }^{111}$ G. Jäkel, ${ }^{178}$ K. B. Jakobi, ${ }^{97}$ K. Jakobs, ${ }^{50}$ T. Jakoubek, ${ }^{176}$ J. Jamieson, ${ }^{55}$ K. W. Janas, ${ }^{81 a}$ G. Jarlskog, ${ }^{94}$ A. E. Jaspan,${ }^{88}$ N. Javadov, ${ }^{77, y}$ T. Javůrek, ${ }^{34}$ M. Javurkova, ${ }^{100}$ F. Jeanneau, ${ }^{141}$ L. Jeanty, ${ }^{128}$ J. Jejelava, ${ }^{156 a}$ P. Jenni, ${ }^{50, \text { aa }}$ S. Jézéquel, ${ }^{4}$ J. Jia, ${ }^{152}$ Z. Jia, ${ }^{13 c}$ Y. Jiang, ${ }^{58 a}$ S. Jiggins, ${ }^{50}$ J. Jimenez Pena, ${ }^{112}$ S. Jin, ${ }^{13 c}$ A. Jinaru, ${ }^{25 b}$ O. Jinnouchi, ${ }^{161}$ H. Jivan, ${ }^{31 f}$ P. Johansson, ${ }^{146}$ K. A. Johns, ${ }^{6}$ C. A. Johnson, ${ }^{63}$ E. Jones, ${ }^{174}$ R. W. L. Jones, ${ }^{87}$ T. J. Jones, ${ }^{88}$ J. Jovicevic, ${ }^{34}$ X. Ju, ${ }^{16}$ J. J. Junggeburth, ${ }^{34}$ A. Juste Rozas, ${ }^{12, u}$ A. Kaczmarska, ${ }^{82}$ M. Kado,${ }^{70 a, 70 b}$ H. Kagan ${ }^{124}$ M. Kagan, ${ }^{150}$ A. Kahn, ${ }^{37}$ C. Kahra, ${ }^{97}$ T. Kaji ${ }^{175}$ E. Kajomovitz,${ }^{157}$ C. W. Kalderon, ${ }^{27}$ A. Kaluza, ${ }^{97}$ A. Kamenshchikov, ${ }^{120}$ M. Kaneda, ${ }^{160}$

N. J. Kang, ${ }^{142}$ S. Kang, ${ }^{76}$ Y. Kano, ${ }^{114}$ J. Kanzaki, ${ }^{79}$ D. Kar, ${ }^{31 \mathrm{f}}$ K. Karava, ${ }^{131}$ M. J. Kareem, ${ }^{164 \mathrm{~b}}$ I. Karkanias, ${ }^{159}$

S. N. Karpov, ${ }^{77}$ Z. M. Karpova, ${ }^{77}$ V. Kartvelishvili, ${ }^{87}$ A. N. Karyukhin, ${ }^{120}$ E. Kasimi, ${ }^{159}$ C. Kato, ${ }^{58 d}$ J. Katzy, ${ }^{44}$ K. Kawade,${ }^{147}$ K. Kawagoee ${ }^{85}$ T. Kawaguchi,${ }^{114}$ T. Kawamoto, ${ }^{141}$ G. Kawamura, ${ }^{51}$ E. F. Kay, ${ }^{172}$ F. I. Kaya, ${ }^{166}$ S. Kazakos,${ }^{12}$ V. F. Kazanin, ${ }^{119 b, 119 a}$ Y. Ke, ${ }^{152}$ J. M. Keaveney, ${ }^{31 a}$ R. Keeler, ${ }^{172}$ J. S. Keller, ${ }^{32}$ D. Kelsey, ${ }^{153}$ J. J. Kempster, ${ }^{19}$ J. Kendrick, ${ }^{19}$ 
K. E. Kennedy, ${ }^{37}$ O. Kepka, ${ }^{137}$ S. Kersten, ${ }^{178}$ B. P. Kerševan, ${ }^{89}$ S. Ketabchi Haghighat, ${ }^{163}$ M. Khandoga, ${ }^{132}$ A. Khanov, ${ }^{126}$ A. G. Kharlamov, ${ }^{119 b, 119 a}$ T. Kharlamova, ${ }^{119 b, 119 a}$ E. E. Khoda, ${ }^{171}$ T. J. Khoo, ${ }^{17}$ G. Khoriauli, ${ }^{173}$ E. Khramov, ${ }^{77}$ J. Khubua, ${ }^{156 b}$ S. Kido, ${ }^{80}$ M. Kiehn, ${ }^{34}$ A. Kilgallon, ${ }^{128}$ E. Kim, ${ }^{161}$ Y. K. Kim, ${ }^{35}$ N. Kimura, ${ }^{92}$ A. Kirchhoff, ${ }^{51}$ D. Kirchmeier, ${ }^{46}$ J. Kirk, ${ }^{140}$ A. E. Kiryunin, ${ }^{112}$ T. Kishimoto, ${ }^{160}$ D. P. Kisliuk, ${ }^{163}$ V. Kitali, ${ }^{44}$ C. Kitsaki, ${ }^{9}$ O. Kivernyk, ${ }^{22}$ T. Klapdor-Kleingrothaus, ${ }^{50}$ M. Klassen, ${ }^{59}$ C. Klein, ${ }^{32}$ L. Klein, ${ }^{173}$ M. H. Klein, ${ }^{103}$ M. Klein, ${ }^{88}$ U. Klein, ${ }^{88}$ P. Klimek, ${ }^{34}$ A. Klimentov, ${ }^{27}$ F. Klimpel, ${ }^{34}$ T. Klingl, ${ }^{22}$ T. Klioutchnikova, ${ }^{34}$ F. F. Klitzner, ${ }^{111}$ P. Kluit, ${ }^{117}$ S. Kluth, ${ }^{112}$ E. Kneringer, ${ }^{74}$ T. M. Knight, ${ }^{163}$ A. Knue, ${ }^{50}$ D. Kobayashi, ${ }^{85}$ M. Kobel, ${ }^{46}$ M. Kocian, ${ }^{150}$ T. Kodama, ${ }^{160}$ P. Kodys, ${ }^{139}$ D. M. Koeck, ${ }^{153}$ P. T. Koenig, ${ }^{22}$ T. Koffas, ${ }^{32}$ N. M. Köhler, ${ }^{34}$ M. Kolb, ${ }^{141}$ I. Koletsou, ${ }^{4}$ T. Komarek, ${ }^{127}$ K. Köneke, ${ }^{50}$ A. X. Y. Kong, ${ }^{1}$ T. Kono, ${ }^{123}$ V. Konstantinides, ${ }^{92}$ N. Konstantinidis, ${ }^{92}$ B. Konya, ${ }^{94}$ R. Kopeliansky, ${ }^{63}$ S. Koperny, ${ }^{81 a}$ K. Korcyl, ${ }^{82}$ K. Kordas, ${ }^{159}$ G. Koren, ${ }^{158}$ A. Korn, ${ }^{92}$ S. Korn, ${ }^{51}$ I. Korolkov, ${ }^{12}$ E. V. Korolkova, ${ }^{146}$ N. Korotkova, ${ }^{110}$ O. Kortner, ${ }^{112}$ S. Kortner, ${ }^{112}$ V. V. Kostyukhin, ${ }^{146,162}$ A. Kotsokechagia, ${ }^{62}$ A. Kotwal, ${ }^{47}$ A. Koulouris, ${ }^{8}$ A. Kourkoumeli-Charalampidi, ${ }^{68 a, 68 b}$ C. Kourkoumelis, ${ }^{8}$ E. Kourlitis, ${ }^{5}$ R. Kowalewski, ${ }^{172}$ W. Kozanecki, ${ }^{141}$ A. S. Kozhin, ${ }^{120}$ V. A. Kramarenko, ${ }^{110}$ G. Kramberger, ${ }^{89}$ D. Krasnopevtsev, ${ }^{58 a}$ M. W. Krasny, ${ }^{132}$ A. Krasznahorkay, ${ }^{34}$ J. A. Kremer, ${ }^{97}$ J. Kretzschmar, ${ }^{88}$ K. Kreul, ${ }^{17}$ P. Krieger, ${ }^{163}$ F. Krieter, ${ }^{111}$ S. Krishnamurthy, ${ }^{100}$ A. Krishnan, ${ }^{59 b}$ M. Krivos, ${ }^{139}$ K. Krizka, ${ }^{16}$ K. Kroeninger, ${ }^{45}$ H. Kroha, ${ }^{112}$ J. Kroll, ${ }^{137}$ J. Kroll, ${ }^{133}$ K. S. Krowpman, ${ }^{104}$ U. Kruchonak, ${ }^{77}$ H. Krüger, ${ }^{22}$ N. Krumnack, ${ }^{76}$ M. C. Kruse, ${ }^{47}$ J. A. Krzysiak, ${ }^{82}$ A. Kubota, ${ }^{161}$ O. Kuchinskaia, ${ }^{162}$ S. Kuday, ${ }^{3 b}$ D. Kuechler, ${ }^{44}$ J. T. Kuechler, ${ }^{44}$ S. Kuehn, ${ }^{34}$ T. Kuhl, ${ }^{44}$ V. Kukhtin, ${ }^{77}$ Y. Kulchitsky, ${ }^{105, b b}$ S. Kuleshov, ${ }^{143 \mathrm{c}}$ M. Kumar, ${ }^{31 \mathrm{f}}$ N. Kumari, ${ }^{99}$ M. Kuna, ${ }^{56}$ A. Kupco, ${ }^{137}$ T. Kupfer, ${ }^{45}$ O. Kuprash, ${ }^{50}$ H. Kurashige, ${ }^{80}$ L. L. Kurchaninov, ${ }^{164 a}$ Y. A. Kurochkin, ${ }^{105}$ A. Kurova, ${ }^{109}$ M. G. Kurth, ${ }^{13 a, 13 d}$ E. S. Kuwertz, ${ }^{34}$ M. Kuze, ${ }^{161}$ A. K. Kvam, ${ }^{145}$ J. Kvita, ${ }^{127}$ T. Kwan, ${ }^{101}$ C. Lacasta, ${ }^{170}$ F. Lacava, ${ }^{70 a, 70 b}$ H. Lacker, ${ }^{17}$ D. Lacour, ${ }^{132}$ N. N. Lad, ${ }^{92}$ E. Ladygin, ${ }^{77}$ R. Lafaye, ${ }^{4}$ B. Laforge, ${ }^{132}$ T. Lagouri, ${ }^{143 \mathrm{~d}}$ S. Lai, ${ }^{51}$ I. K. Lakomiec, ${ }^{81 a}$ N. Lalloue, ${ }^{56}$ J. E. Lambert, ${ }^{125}$ S. Lammers, ${ }^{63}$ W. Lampl, ${ }^{6}$ C. Lampoudis, ${ }^{159}$ E. Lançon, ${ }^{27}$ U. Landgraf, ${ }^{50}$ M. P. J. Landon, ${ }^{90}$ V. S. Lang, ${ }^{50}$ J. C. Lange, ${ }^{51}$ R. J. Langenberg, ${ }^{100}$ A. J. Lankford, ${ }^{167}$ F. Lanni, ${ }^{27}$ K. Lantzsch, ${ }^{22}$ A. Lanza, ${ }^{68 a}$ A. Lapertosa, ${ }^{53 b, 53 a}$ J. F. Laporte, ${ }^{141}$ T. Lari, ${ }^{66 a}$ F. Lasagni Manghi, ${ }^{21 b, 21 a}$ M. Lassnig, ${ }^{34}$ V. Latonova, ${ }^{137}$ T. S. Lau, ${ }^{60 a}$ A. Laudrain, ${ }^{97}$ A. Laurier, ${ }^{32}$ M. Lavorgna, ${ }^{67 a, 67 b}$ S. D. Lawlor, ${ }^{91}$ M. Lazzaroni, ${ }^{66 a, 66 b}$ B. Le, ${ }^{98}$ A. Lebedev, ${ }^{76}$ M. LeBlanc, ${ }^{34}$ T. LeCompte, ${ }^{5}$ F. Ledroit-Guillon, ${ }^{56}$ A. C. A. Lee, ${ }^{92}$ C. A. Lee, ${ }^{27}$ G. R. Lee, ${ }^{15}$ L. Lee,${ }^{57}$ S. C. Lee, ${ }^{155}$ S. Lee ${ }^{76}$ L. L. Leeuw, ${ }^{31 c}$ B. Lefebvre, ${ }^{164 a}$ H. P. Lefebvre, ${ }^{91}$ M. Lefebvre, ${ }^{172}$ C. Leggett, ${ }^{16}$ K. Lehmann, ${ }^{149}$ N. Lehmann, ${ }^{18}$ G. Lehmann Miotto, ${ }^{34}$ W. A. Leight, ${ }^{44}$ A. Leisos, ${ }^{159, c c}$ M. A. L. Leite, ${ }^{78 c}$ C. E. Leitgeb, ${ }^{44}$ R. Leitner, ${ }^{139}$ K. J. C. Leney, ${ }^{40}$ T. Lenz, ${ }^{22}$ S. Leone, ${ }^{69}$ C. Leonidopoulos, ${ }^{48}$ A. Leopold, ${ }^{132}$ C. Leroy, ${ }^{107}$ R. Les, ${ }^{104}$ C. G. Lester, ${ }^{30}$ M. Levchenko, ${ }^{134}$ J. Levêque, ${ }^{4}$ D. Levin, ${ }^{103}$ L. J. Levinson, ${ }^{176}$ D. J. Lewis, ${ }^{19}$ B. Li, ${ }^{13 b}$

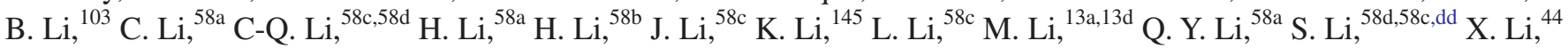
Y. Li ${ }^{44}$ Z. Li, ${ }^{58 b}$ Z. Li, ${ }^{131}$ Z. Li, ${ }^{101}$ Z. Li, ${ }^{88}$ Z. Liang, ${ }^{13 a}$ M. Liberatore, ${ }^{44}$ B. Liberti, ${ }^{71 a}$ K. Lie, ${ }^{60 c}$ K. Lin, ${ }^{104}$ R. A. Linck, ${ }^{63}$ R. E. Lindley, ${ }^{6}$ J. H. Lindon, ${ }^{2}$ A. Linss, ${ }^{44}$ A. L. Lionti, ${ }^{52}$ E. Lipeles, ${ }^{133}$ A. Lipniacka, ${ }^{15}$ T. M. Liss, ${ }^{169, \text { ee }}$ A. Lister, ${ }^{171}$ J. D. Little, ${ }^{7}$ B. Liu, ${ }^{13 a}$ B. X. Liu, ${ }^{149}$ J. B. Liu, ${ }^{58 a}$ J. K. K. Liu, ${ }^{35}$ K. Liu, ${ }^{58 d, 58 c}$ M. Liu, ${ }^{58 a}$ M. Y. Liu, ${ }^{58 a}$ P. Liu, ${ }^{13 a}$ X. Liu, ${ }^{58 a}$ Y. Liu, ${ }^{44}$ Y. Liu, ${ }^{13 c, 13 d}$ Y. L. Liu, ${ }^{103}$ Y. W. Liu, ${ }^{58 a}$ M. Livan, ${ }^{68 a, 68 b}$ A. Lleres, ${ }^{56}$ J. Llorente Merino, ${ }^{149}$ S. L. Lloyd, ${ }^{90}$ E. M. Lobodzinska, ${ }^{44}$ P. Loch, ${ }^{6}$ S. Loffredo, ${ }^{71 \mathrm{a}, 71 \mathrm{~b}}$ T. Lohse, ${ }^{17}$ K. Lohwasser, ${ }^{146}$ M. Lokajicek, ${ }^{137}$ J. D. Long, ${ }^{169}$ R. E. Long, ${ }^{87}$ I. Longarini, ${ }^{70 a, 70 b}$ L. Longo, ${ }^{34}$ R. Longo, ${ }^{169}$ I. Lopez Paz, ${ }^{12}$ A. Lopez Solis, ${ }^{44}$ J. Lorenz, ${ }^{111}$ N. Lorenzo Martinez, A. M. Lory, ${ }^{111}$ A. Lösle, ${ }^{50}$ X. Lou, ${ }^{43 a, 43 b}$ X. Lou, ${ }^{13 a}$ A. Lounis, ${ }^{62}$ J. Love, ${ }^{5}$ P. A. Love, ${ }^{87}$ J. J. Lozano Bahilo, ${ }^{170}$ G. Lu, ${ }^{13 a}$ M. Lu, ${ }^{58 \mathrm{a}}$ S. Lu, ${ }^{133}$ Y. J. Lu, ${ }^{61}$ H. J. Lubatti, ${ }^{145}$ C. Luci, ${ }^{70 a, 70 b}$ F. L. Lucio Alves, ${ }^{13 c}$ A. Lucotte, ${ }^{56}$ F. Luehring, ${ }^{63}$ I. Luise, ${ }^{152}$ L. Luminari, ${ }^{70 a}$ B. Lund-Jensen, ${ }^{151}$ N. A. Luongo, ${ }^{128}$ M. S. Lutz, ${ }^{158}$ D. Lynn, ${ }^{27}$ H. Lyons, ${ }^{88}$ R. Lysak, ${ }^{137}$ E. Lytken, ${ }^{94}$ F. Lyu, ${ }^{13 a}$ V. Lyubushkin, ${ }^{77}$ T. Lyubushkina, ${ }^{77}$ H. Ma, ${ }^{27}$ L. L. Ma, ${ }^{58 b}$ Y. Ma, ${ }^{92}$ D. M. Mac Donell, ${ }^{172}$ G. Maccarrone, ${ }^{49}$ C. M. Macdonald, ${ }^{146}$ J. C. MacDonald, ${ }^{146}$ R. Madar, ${ }^{36}$ W. F. Mader, ${ }^{46}$ M. Madugoda Ralalage Don, ${ }^{126}$ N. Madysa, ${ }^{46}$

J. Maeda, ${ }^{80}$ T. Maeno, ${ }^{27}$ M. Maerker, ${ }^{46}$ V. Magerl, ${ }^{50}$ J. Magro, ${ }^{64 a, 64 c}$ D. J. Mahon, ${ }^{37}$ C. Maidantchik, ${ }^{78 b}$ A. Maio, ${ }^{136 a, 136 b, 136 d}$ K. Maj, ${ }^{81 \mathrm{a}}$ O. Majersky, ${ }^{26 a}$ S. Majewski, ${ }^{128}$ N. Makovec, ${ }^{62}$ B. Malaescu, ${ }^{132}$ Pa. Malecki, ${ }^{82}$ V. P. Maleev, ${ }^{134}$ F. Malek, ${ }^{56}$ D. Malito, ${ }^{39 b, 39 a}$ U. Mallik, ${ }^{75}$ C. Malone, ${ }^{30}$ S. Maltezos, ${ }^{9}$ S. Malyukov, ${ }^{77}$ J. Mamuzic, ${ }^{170}$ G. Mancini, ${ }^{49}$ J. P. Mandalia, ${ }^{90}$ I. Mandić, ${ }^{89}$ L. Manhaes de Andrade Filho, ${ }^{78 a}$ I. M. Maniatis, ${ }^{159}$ M. Manisha, ${ }^{141}$ J. Manjarres Ramos, ${ }^{46}$ K. H. Mankinen, ${ }^{94}$ A. Mann, ${ }^{111}$ A. Manousos, ${ }^{74}$ B. Mansoulie, ${ }^{141}$ I. Manthos, ${ }^{159}$ S. Manzoni, ${ }^{117}$ A. Marantis, ${ }^{159, c c}$ L. Marchese, ${ }^{131}$ G. Marchiori, ${ }^{132}$ M. Marcisovsky, ${ }^{137}$ L. Marcoccia, ${ }^{71 a, 71 b}$ C. Marcon, ${ }^{94}$ M. Marjanovic, ${ }^{125}$ Z. Marshall, ${ }^{16}$ S. Marti-Garcia, ${ }^{170}$

T. A. Martin, ${ }^{174}$ V. J. Martin, ${ }^{48}$ B. Martin dit Latour, ${ }^{15}$ L. Martinelli, ${ }^{72 a, 72 b}$ M. Martinez, ${ }^{12, u}$ P. Martinez Agullo, ${ }^{170}$

V. I. Martinez Outschoorn, ${ }^{100}$ S. Martin-Haugh, ${ }^{140}$ V. S. Martoiu, ${ }^{25 b}$ A. C. Martyniuk, ${ }^{92}$ A. Marzin, ${ }^{34}$ S. R. Maschek, ${ }^{112}$ L. Masetti, ${ }^{97}$ T. Mashimo, ${ }^{160}$ R. Mashinistov, ${ }^{108}$ J. Masik, ${ }^{98}$ A. L. Maslennikov, ${ }^{119 b, 119 a}$ L. Massa, ${ }^{21 b, 21 a}$ P. Massarotti, ${ }^{67 a, 67 b}$ 
P. Mastrandrea, ${ }^{69 a, 69 \mathrm{~b}}$ A. Mastroberardino, ${ }^{39 \mathrm{~b}, 39 \mathrm{a}}$ T. Masubuchi, ${ }^{160}$ D. Matakias, ${ }^{27}$ T. Mathisen, ${ }^{168}$ A. Matic, ${ }^{111}$ N. Matsuzawa, ${ }^{160}$ J. Maurer, ${ }^{25 b}$ B. Maček, ${ }^{89}$ D. A. Maximov, ${ }^{119 b, 119 a}$ R. Mazini, ${ }^{155}$ I. Maznas, ${ }^{159}$ S. M. Mazza, ${ }^{142}$ C. Mc Ginn, ${ }^{27}$ J. P. Mc Gowan, ${ }^{101}$ S. P. Mc Kee, ${ }^{103}$ T. G. McCarthy, ${ }^{12}$ W. P. McCormack, ${ }^{16}$ E. F. McDonald, ${ }^{102}$ A. E. McDougall, ${ }^{117}$ J. A. Mcfayden, ${ }^{153}$ G. Mchedlidze, ${ }^{156 b}$ M. A. McKay,${ }^{40}$ K. D. McLean, ${ }^{172}$ S. J. McMahon, ${ }^{140}$ P. C. McNamara, ${ }^{102}$ R. A. McPherson, ${ }^{172, m}$ J. E. Mdhluli, ${ }^{31 f}$ Z. A. Meadows, ${ }^{100}$ S. Meehan, ${ }^{34}$ T. Megy, ${ }^{36}$ S. Mehlhase, ${ }^{111}$ A. Mehta, ${ }^{88}$ B. Meirose, ${ }^{41}$ D. Melini, ${ }^{157}$ B. R. Mellado Garcia, ${ }^{31 f}$ F. Meloni, ${ }^{44}$ A. Melzer, ${ }^{22}$ E. D. Mendes Gouveia, ${ }^{136 a}$

A. M. Mendes Jacques Da Costa, ${ }^{19}$ H. Y. Meng, ${ }^{163}$ L. Meng, ${ }^{34}$ S. Menke, ${ }^{112}$ M. Mentink, ${ }^{34}$ E. Meoni, ${ }^{39 b, 39 a}$ S. A. M. Merkt, ${ }^{135}$ C. Merlassino, ${ }^{131}$ P. Mermod, ${ }^{52, a}$ L. Merola, ${ }^{67,67 b}$ C. Meroni, ${ }^{66 a}$ G. Merz, ${ }^{103}$ O. Meshkov, ${ }^{110,108}$ J. K. R. Meshreki, ${ }^{148}$ J. Metcalfe, ${ }^{5}$ A. S. Mete ${ }^{5}$ C. Meyer, ${ }^{63}$ J-P. Meyer,${ }^{141}$ M. Michetti, ${ }^{17}$ R. P. Middleton, ${ }^{140}$ L. Mijović, ${ }^{48}$ G. Mikenberg, ${ }^{176}$ M. Mikestikova, ${ }^{137}$ M. Mikuž ${ }^{89}$ H. Mildner, ${ }^{146}$ A. Milic, ${ }^{163}$ C. D. Milke, ${ }^{40}$ D. W. Miller, ${ }^{35}$ L. S. Miller, ${ }^{32}$ A. Milov, ${ }^{176}$ D. A. Milstead, ${ }^{43,43 b}$ A. A. Minaenko, ${ }^{120}$ I. A. Minashvili, ${ }^{156 b}$ L. Mince, ${ }^{55}$ A. I. Mincer,${ }^{122}$ B. Mindur, ${ }^{81 a}$ M. Mineev, ${ }^{77}$ Y. Minegishi, ${ }^{160}$ Y. Mino,${ }^{83}$ L. M. Mir ${ }^{12}$ M. Miralles Lopez, ${ }^{170}$ M. Mironova, ${ }^{131}$ T. Mitani, ${ }^{175}$ V. A. Mitsou, ${ }^{170}$ M. Mittal ${ }^{58 c}$ O. Miu, ${ }^{163}$ P. S. Miyagawa, ${ }^{90}$ Y. Miyazaki, ${ }^{85}$ A. Mizukami, ${ }^{79}$ J. U. Mjörnmark,${ }^{94}$ T. Mkrtchyan, ${ }^{59 a}$ M. Mlynarikova, ${ }^{118}$ T. Moa, ${ }^{43 a, 43 b}$ S. Mobius,${ }^{51}$ K. Mochizuki, ${ }^{107}$ P. Moder, ${ }^{44}$ P. Mogg, ${ }^{111}$ S. Mohapatra,${ }^{37}$ G. Mokgatitswane, ${ }^{31 \mathrm{f}}$ B. Mondal, ${ }^{148}$ S. Mondal, ${ }^{138}$ K. Mönig, ${ }^{44}$ E. Monnier, ${ }^{99}$ A. Montalbano, ${ }^{149}$ J. Montejo Berlingen, ${ }^{34}$ M. Montella, ${ }^{124}$ F. Monticelli, ${ }^{86}$ N. Morange, ${ }^{62}$ A. L. Moreira De Carvalho, ${ }^{136 a}$ M. Moreno Llácer, ${ }^{170}$ C. Moreno Martinez, ${ }^{12}$ P. Morettini, ${ }^{53 \mathrm{~b}}$ M. Morgenstern, ${ }^{157}$ S. Morgenstern, ${ }^{174}$ D. Mori, ${ }^{149}$ M. Morii,${ }^{57}$ M. Morinaga, ${ }^{160}$ V. Morisbak, ${ }^{130}$ A. K. Morley, ${ }^{34}$ A. P. Morris, ${ }^{92}$ L. Morvaj, ${ }^{34}$ P. Moschovakos, ${ }^{34}$ B. Moser, ${ }^{117}$ M. Mosidze, ${ }^{156 b}$ T. Moskalets, ${ }^{50}$ P. Moskvitina, ${ }^{16}$ J. Moss, ${ }^{29, f f}$ E. J. W. Moyse, ${ }^{100}$ S. Muanza, ${ }^{99}$ J. Mueller, ${ }^{135}$ D. Muenstermann, ${ }^{87}$ G. A. Mullier, ${ }^{94}$ J. J. Mullin, ${ }^{133}$ D. P. Mungo, ${ }^{66,66 \mathrm{~b}}$ J. L. Munoz Martinez, ${ }^{12}$ F. J. Munoz Sanchez, ${ }^{98}$ M. Murin, ${ }^{98}$ P. Murin, ${ }^{26 \mathrm{~b}}$ W. J. Murray, ${ }^{174,140}$ A. Murrone, ${ }^{66 a, 66 b}$ J. M. Muse, ${ }^{125}$ M. Muškinja, ${ }^{16}$ C. Mwewa, ${ }^{27}$ A. G. Myagkov, ${ }^{120, i}$ A. A. Myers,${ }^{135}$ G. Myers, ${ }^{63}$ J. Myers, ${ }^{128}$ M. Myska, ${ }^{138}$ B. P. Nachman, ${ }^{16}$ O. Nackenhorst, ${ }^{45}$ A. Nag Nag, ${ }^{46}$ K. Nagai,${ }^{131}$ K. Nagano, ${ }^{79}$ J. L. Nagle,${ }^{27}$ E. Nagy, ${ }^{99}$ A. M. Nairz ${ }^{34}$ Y. Nakahama, ${ }^{114}$ K. Nakamura, ${ }^{79}$ H. Nanjo, ${ }^{129}$ F. Napolitano, ${ }^{59 a}$ R. Narayan, ${ }^{40}$ I. Naryshkin, ${ }^{134}$ M. Naseri, ${ }^{32}$ C. Nass, ${ }^{22}$ T. Naumann, ${ }^{44}$ G. Navarro, ${ }^{20 a}$ J. Navarro-Gonzalez, ${ }^{170}$ P. Y. Nechaeva, ${ }^{108}$ F. Nechansky, ${ }^{44}$ T. J. Neep, ${ }^{19}$ A. Negri, ${ }^{68 a, 68 b}$ M. Negrini, ${ }^{21 b}$ C. Nellist, ${ }^{116}$ C. Nelson, ${ }^{101}$ K. Nelson, ${ }^{103}$ M. E. Nelson, ${ }^{43 a, 43 b}$ S. Nemecek, ${ }^{137}$ M. Nessi, ${ }^{34, g g}$ M. S. Neubauer, ${ }^{169}$ F. Neuhaus, ${ }^{97}$ J. Neundorf, ${ }^{44}$ R. Newhouse, ${ }^{171}$ P. R. Newman, ${ }^{19}$ C. W. Ng, ${ }^{135}$ Y. S. Ng, ${ }^{17}$ Y. W. Y. Ng, ${ }^{167}$ B. Ngair, ${ }^{33 e}$ H. D. N. Nguyen, ${ }^{99}$ T. Nguyen Manh, ${ }^{107}$ R. B. Nickerson, ${ }^{131}$ R. Nicolaidou, ${ }^{141}$ D. S. Nielsen, ${ }^{38}$ J. Nielsen, ${ }^{142}$ M. Niemeyer, ${ }^{51}$ N. Nikiforou, ${ }^{10}$ V. Nikolaenko, ${ }^{120, i}$ I. Nikolic-Audit, ${ }^{132}$ K. Nikolopoulos, ${ }^{19}$ P. Nilsson, ${ }^{27}$ H. R. Nindhito, ${ }^{52}$ A. Nisati, ${ }^{70 a}$ N. Nishu, ${ }^{2}$ R. Nisius, ${ }^{12}$ T. Nitta, ${ }^{175}$ T. Nobe, ${ }^{160}$ D. L. Noel, ${ }^{30}$

Y. Noguchi, ${ }^{83}$ I. Nomidis, ${ }^{132}$ M. A. Nomura, ${ }^{27}$ M. B. Norfolk, ${ }^{146}$ R. R. B. Norisam, ${ }^{92}$ J. Novak, ${ }^{89}$ T. Novak, ${ }^{44}$ O. Novgorodova, ${ }^{46}$ L. Novotny, ${ }^{138}$ R. Novotny, ${ }^{115}$ L. Nozka, ${ }^{127}$ K. Ntekas, ${ }^{167}$ E. Nurse, ${ }^{92}$ F. G. Oakham, ${ }^{32, e}$ J. Ocariz, ${ }^{132}$ A. Ochi, ${ }^{80}$ I. Ochoa, ${ }^{136 a}$ J. P. Ochoa-Ricoux ${ }^{143 a}$ K. O'Connor, ${ }^{24}$ S. Oda,${ }^{85}$ S. Odaka, ${ }^{79}$ S. Oerdek,${ }^{51}$ A. Ogrodnik,${ }^{81 a}$ A. Oh,${ }^{98}$ C. C. Ohm, ${ }^{151}$ H. Oide, ${ }^{161}$ R. Oishi, ${ }^{160}$ M. L. Ojeda, ${ }^{163}$ Y. Okazaki, ${ }^{83}$ M. W. O'Keefe, ${ }^{88}$ Y. Okumura, ${ }^{160}$ A. Olariu, ${ }^{25 b}$ L. F. Oleiro Seabra, ${ }^{136 a}$ S. A. Olivares Pino, ${ }^{143 \mathrm{~d}}$ D. Oliveira Damazio, ${ }^{27}$ D. Oliveira Goncalves,${ }^{78 a}$ J. L. Oliver,${ }^{1}$ M. J. R. Olsson, ${ }^{167}$ A. Olszewski, ${ }^{82}$ J. Olszowska, ${ }^{82}$ Ö. O. Öncel, ${ }^{22}$ D. C. O’Neil, ${ }^{149}$ A. P. O’neill, ${ }^{131}$ A. Onofre, ${ }^{136 a, 136 e}$ P. U.E. Onyisi, ${ }^{10}$ H. Oppen, ${ }^{130}$ R. G. Oreamuno Madriz, ${ }^{118}$ M. J. Oreglia, ${ }^{35}$ G. E. Orellana, ${ }^{86}$ D. Orestano, ${ }^{72 a, 72 b}$ N. Orlando, ${ }^{12}$ R. S. Orr, ${ }^{163}$ V. O'Shea, ${ }^{55}$ R. Ospanov, ${ }^{58 a}$ G. Otero y Garzon, ${ }^{28}$ H. Otono, ${ }^{85}$ P. S. Ott,,${ }^{59}$ G. J. Ottino, ${ }^{16}$ M. Ouchrif, ${ }^{33 \mathrm{~d}}$ J. Ouellette, ${ }^{27}$ F. Ould-Saada, ${ }^{130}$ A. Ouraou, ${ }^{141, a}$ Q. Ouyang, ${ }^{13 \mathrm{a}}$ M. Owen, ${ }^{55}$ R. E. Owen, ${ }^{140}$ V. E. Ozcan, ${ }^{11 \mathrm{c}}$ N. Ozturk, ${ }^{7}$ S. Ozturk, ${ }^{11 \mathrm{c}}$ J. Pacalt, ${ }^{127}$ H. A. Pacey, ${ }^{30}$ K. Pachal,${ }^{47}$ A. Pacheco Pages, ${ }^{12}$ C. Padilla Aranda, ${ }^{12}$ S. Pagan Griso, ${ }^{16}$ G. Palacino, ${ }^{63}$ S. Palazzo, ${ }^{48}$ S. Palestini, ${ }^{34}$ M. Palka,${ }^{81 b}$ P. Palni, ${ }^{81 a}$ D. K. Panchal, ${ }^{10}$ C. E. Pandini,${ }^{52}$ J. G. Panduro Vazquez, ${ }^{91}$

P. Pani ${ }^{44}$ G. Panizzo, ${ }^{64 a, 64 c}$ L. Paolozzi,${ }^{52}$ C. Papadatos ${ }^{107}$ S. Parajuli, ${ }^{40}$ A. Paramonov, ${ }^{5}$ C. Paraskevopoulos, ${ }^{9}$ D. Paredes Hernandez, ${ }^{60 b}$ S. R. Paredes Saenz, ${ }^{131}$ B. Parida, ${ }^{176}$ T. H. Park, ${ }^{163}$ A. J. Parker ${ }^{29}$ M. A. Parker,${ }^{30}$ F. Parodi, ${ }^{53 b, 53 a}$ E. W. Parrish, ${ }^{118}$ J. A. Parsons, ${ }^{37}$ U. Parzefall ${ }^{50}$ L. Pascual Dominguez, ${ }^{158}$ V. R. Pascuzzi, ${ }^{16}$ F. Pasquali, ${ }^{117}$ E. Pasqualucci, ${ }^{70 a}$ S. Passaggio, ${ }^{53 \mathrm{~b}}$ F. Pastore, ${ }^{91}$ P. Pasuwan, ${ }^{43,43 \mathrm{~b}}$ J. R. Pater, ${ }^{98}$ A. Pathak, ${ }^{177}$ J. Patton, ${ }^{88}$ T. Pauly, ${ }^{34} \mathrm{~J}$. Pearkes, ${ }^{150}$ M. Pedersen, ${ }^{130}$ L. Pedraza Diaz, ${ }^{116}$ R. Pedro, ${ }^{16 a}$ T. Peiffer ${ }^{51}$ S. V. Peleganchuk, ${ }^{119 b, 119 a}$ O. Penc, ${ }^{138}$ C. Peng, ${ }^{60 b}$ H. Peng, ${ }^{58 a}$ M. Penzin, ${ }^{163}$ B. S. Peralva, ${ }^{78 a}$ M. M. Perego, ${ }^{62}$ A. P. Pereira Peixoto, ${ }^{16 a}$ L. Pereira Sanchez, ${ }^{43 a, 43 b}$ D. V. Perepelitsa, ${ }^{27}$

E. Perez Codina, ${ }^{164 a}$ M. Perganti, ${ }^{9}$ L. Perini,${ }^{66 a, 66 b}$ H. Pernegger,${ }^{34}$ S. Perrella, ${ }^{34}$ A. Perrevoort, ${ }^{117}$ K. Peters, ${ }^{44}$ R. F. Y. Peters, ${ }^{98}$ B. A. Petersen, ${ }^{34}$ T. C. Petersen, ${ }^{38}$ E. Petit, ${ }^{99}$ V. Petousis, ${ }^{138}$ C. Petridou, ${ }^{159}$ P. Petroff, ${ }^{62}$ F. Petrucci, ${ }^{72 a, 72 b}$ M. Pettee,${ }^{179}$ N. E. Pettersson, ${ }^{34}$ K. Petukhova, ${ }^{139}$ A. Peyaud, ${ }^{141}$ R. Pezoa,${ }^{143 e}$ L. Pezzotti, ${ }^{68 a, 68 b}$ G. Pezzullo, ${ }^{179}$ T. Pham, ${ }^{102}$ 
P. W. Phillips, ${ }^{140}$ M. W. Phipps, ${ }^{169}$ G. Piacquadio, ${ }^{152}$ E. Pianori, ${ }^{16}$ F. Piazza, ${ }^{66 a, 66 b}$ A. Picazio, ${ }^{100}$ R. Piegaia,${ }^{28}$ D. Pietreanu, ${ }^{25 b}$ J. E. Pilcher, ${ }^{35}$ A. D. Pilkington, ${ }^{98}$ M. Pinamonti, ${ }^{64 a, 64 c}$ J. L. Pinfold, ${ }^{2}$ C. Pitman Donaldson, ${ }^{92}$ D. A. Pizzi, ${ }^{32}$ L. Pizzimento, ${ }^{71 \mathrm{a}, 71 \mathrm{~b}}$ A. Pizzini, ${ }^{117}$ M.-A. Pleier, ${ }^{27}$ V. Plesanovs,${ }^{50}$ V. Pleskot, ${ }^{139}$ E. Plotnikova, ${ }^{77}$ P. Podberezko, ${ }^{119 b, 119 a}$ R. Poettgen, ${ }^{94}$ R. Poggi ${ }^{52}$ L. Poggioli, ${ }^{132}$ I. Pogrebnyak, ${ }^{104}$ D. Pohl,${ }^{22}$ I. Pokharel,${ }^{51}$ G. Polesello, ${ }^{68 a}$ A. Poley, ${ }^{149,164 a}$ A. Policicchio, ${ }^{70 a, 70 b}$ R. Polifka, ${ }^{139}$ A. Polini,${ }^{21 b}$ C. S. Pollard, ${ }^{44}$ Z. B. Pollock, ${ }^{124}$ V. Polychronakos, ${ }^{27}$ D. Ponomarenko, ${ }^{109}$ L. Pontecorvo, ${ }^{34}$ S. Popa ${ }^{25 a}$ G. A. Popeneciu, ${ }^{25 d}$ L. Portales, ${ }^{4}$ D. M. Portillo Quintero ${ }^{56}$ S. Pospisil,${ }^{138}$ P. Postolache,${ }^{25 c}$ K. Potamianos, ${ }^{131}$ I. N. Potrap, ${ }^{77}$ C. J. Potter,${ }^{30}$ H. Potti, ${ }^{1}$ T. Poulsen,${ }^{44}$ J. Poveda, ${ }^{170}$ T. D. Powell,${ }^{146}$ G. Pownall, ${ }^{44}$ M. E. Pozo Astigarraga ${ }^{34}$ A. Prades Ibanez, ${ }^{170}$ P. Pralavorio, ${ }^{99}$ M. M. Prapa,${ }^{42}$ S. Prell, ${ }^{76}$ D. Price, ${ }^{98}$ M. Primavera,${ }^{65 a}$ M. A. Principe Martin, ${ }^{96}$ M. L. Proffitt, ${ }^{145}$ N. Proklova, ${ }^{109}$ K. Prokofiev, ${ }^{60 c}$ F. Prokoshin, ${ }^{77}$ S. Protopopescu, ${ }^{27}$ J. Proudfoot, ${ }^{5}$ M. Przybycien ${ }^{81 a}$ D. Pudzha, ${ }^{134}$ P. Puzo,${ }^{62}$ D. Pyatiizbyantseva, ${ }^{109}$ J. Qian, ${ }^{103}$ Y. Qin, ${ }^{98}$ A. Quadt, ${ }^{51}$ M. Queitsch-Maitland,${ }^{34}$ G. Rabanal Bolanos, ${ }^{57}$ F. Ragusa, ${ }^{66 a, 66 b}$ G. Rahal, ${ }^{95}$ J. A. Raine, ${ }^{52}$ S. Rajagopalan, ${ }^{27}$ K. Ran,${ }^{13 a, 13 d}$ D. F. Rassloff, ${ }^{59 a}$

D. M. Rauch, ${ }^{44}$ S. Rave, ${ }^{97}$ B. Ravina,${ }^{55}$ I. Ravinovich, ${ }^{176}$ M. Raymond ${ }^{34}$ A. L. Read, ${ }^{130}$ N. P. Readioff, ${ }^{146}$

D. M. Rebuzzi, ${ }^{68 \mathrm{a}, 68 \mathrm{~b}}$ G. Redlinger, ${ }^{27}$ K. Reeves ${ }^{41}$ D. Reikher ${ }^{158}$ A. Reiss, ${ }^{97}$ A. Rej, ${ }^{148}$ C. Rembser ${ }^{34}$ A. Renardi, ${ }^{44}$ M. Renda, ${ }^{25 b}$ M. B. Rendel, ${ }^{112}$ A. G. Rennie, ${ }^{55}$ S. Resconi, ${ }^{66 a}$ E. D. Resseguie, ${ }^{16}$ S. Rettie, ${ }^{92}$ B. Reynolds, ${ }^{124}$ E. Reynolds, ${ }^{19}$ M. Rezaei Estabragh, ${ }^{178}$ O. L. Rezanova, ${ }^{19 b, 119 \mathrm{a}}$ P. Reznicek, ${ }^{139}$ E. Ricci, ${ }^{73 a, 73 b}$ R. Richter, ${ }^{112}$ S. Richter, ${ }^{44}$

E. Richter-Was, ${ }^{81 b}$ M. Ridel ${ }^{133}{ }^{13}$ P. Rieck, ${ }^{112}$ P. Riedler, ${ }^{34}$ O. Rifki, ${ }^{44}$ M. Rijssenbeek ${ }^{152}$ A. Rimoldi, ${ }^{68 a, 68 b}$ M. Rimoldi, ${ }^{44}$ L. Rinaldi, ${ }^{21 b}$ T. T. Rinn, ${ }^{169}$ M. P. Rinnagel, ${ }^{111}$ G. Ripellino, ${ }^{151}$ I. Riu, ${ }^{12}$ P. Rivadeneira, ${ }^{44}$ J. C. Rivera Vergara, ${ }^{172}$

F. Rizatdinova, ${ }^{126}$ E. Rizvi,${ }^{90}$ C. Rizzi,${ }^{52}$ B. A. Roberts, ${ }^{174}$ S. H. Robertson, ${ }^{101, m}$ M. Robin, ${ }^{44}$ D. Robinson, ${ }^{30}$ C. M. Robles Gajardo, ${ }^{143 \mathrm{e}}$ M. Robles Manzano,${ }^{97}$ A. Robson, ${ }^{55}$ A. Rocchi,${ }^{71 a, 71 b}$ C. Roda,${ }^{69 a, 69 b}$ S. Rodriguez Bosca,${ }^{59 a}$ A. Rodriguez Rodriguez, ${ }^{50}$ A. M. Rodríguez Vera ${ }^{164 b}$ S. Roe, ${ }^{34}$ J. Roggel, ${ }^{178}$ O. Røhne, ${ }^{130}$ R. A. Rojas, ${ }^{143}$ B. Roland, ${ }^{50}$ C. P. A. Roland, ${ }^{63}$ J. Roloff, ${ }^{27}$ A. Romaniouk, ${ }^{109}$ M. Romano, ${ }^{21 b, 21 \mathrm{a}}$ N. Rompotis, ${ }^{88}$ M. Ronzani, ${ }^{122}$ L. Roos, ${ }^{132}$ S. Rosati, ${ }^{70 a}$ G. Rosin, ${ }^{100}$ B. J. Rosser, ${ }^{133}$ E. Rossi, ${ }^{163}$ E. Rossi, ${ }^{4}$ E. Rossi, ${ }^{67 a, 67 b}$ L. P. Rossi, ${ }^{53 b}$ L. Rossini, ${ }^{44}$ R. Rosten, ${ }^{124}$ M. Rotaru, ${ }^{25 b}$ B. Rottler, ${ }^{50}$ D. Rousseau, ${ }^{62}$ D. Rousso,${ }^{30}$ G. Rovelli, ${ }^{68 a, 68 b}$ A. Roy, ${ }^{10}$ A. Rozanov, ${ }^{99}$ Y. Rozen, ${ }^{157}$ X. Ruan,${ }^{31 f}$ A. J. Ruby, ${ }^{88}$ T. A. Ruggeri, ${ }^{1}$ F. Rühr, ${ }^{50}$ A. Ruiz-Martinez, ${ }^{170}$ A. Rummler, ${ }^{34}$ Z. Rurikova,${ }^{50}$ N. A. Rusakovich,${ }^{77}$ H. L. Russell, ${ }^{34}$ L. Rustige, ${ }^{36}$ J. P. Rutherfoord, ${ }^{6}$ E. M. Rüttinger, ${ }^{146}$ M. Rybar, ${ }^{139}$ E. B. Rye, ${ }^{130}$ A. Ryzhov, ${ }^{120}$ J. A. Sabater Iglesias ${ }^{44}$ P. Sabatini, ${ }^{170}$ L. Sabetta, ${ }^{70 a, 70 b}$ H. F-W. Sadrozinski, ${ }^{142}$ R. Sadykov, ${ }^{77}$ F. Safai Tehrani, ${ }^{70 a}$ B. Safarzadeh Samani, ${ }^{153}$ M. Safdari, ${ }^{150}$ P. Saha, ${ }^{118}$ S. Saha ${ }^{101}$ M. Sahinsoy, ${ }^{112}$ A. Sahu, ${ }^{178}$ M. Saimpert, ${ }^{141}$ M. Saito, ${ }^{160}$ T. Saito, ${ }^{160}$ D. Salamani, ${ }^{52}$ G. Salamanna ${ }^{72 a, 72 b}$ A. Salnikov ${ }^{150}$ J. Salt, ${ }^{170}$ A. Salvador Salas, ${ }^{12}$ D. Salvatore, ${ }^{39 b, 39 a}$ F. Salvatore, ${ }^{153}$ A. Salzburger, ${ }^{34}$ D. Sammel, ${ }^{50}$ D. Sampsonidis, ${ }^{159}$ D. Sampsonidou, ${ }^{58 d, 58 c}$ J. Sánchez,${ }^{170}$ A. Sanchez Pineda, ${ }^{4}$ V. Sanchez Sebastian, ${ }^{170}$ H. Sandaker, ${ }^{130}$ C. O. Sander, ${ }^{44}$ I. G. Sanderswood, ${ }^{87}$ J. A. Sandesara, ${ }^{100}$ M. Sandhoff, ${ }^{178}$ C. Sandoval, ${ }^{20 b}$ D. P. C. Sankey, ${ }^{140}$ M. Sannino, ${ }^{53 b, 53 a}$ Y. Sano, ${ }^{114}$ A. Sansoni, ${ }^{49}$ C. Santoni, ${ }^{36}$ H. Santos, ${ }^{136 a, 136 b}$ S. N. Santpur, ${ }^{16}$ A. Santra, ${ }^{176}$ K. A. Saoucha, ${ }^{146}$ A. Sapronov, ${ }^{77}$ J. G. Saraiva, ${ }^{136 a, 136 \mathrm{~d}}$ O. Sasaki, ${ }^{79}$ K. Sato, ${ }^{165}$ C. Sauer, ${ }^{59 b}$ F. Sauerburger, ${ }^{50}$ E. Sauvan, ${ }^{4}$ P. Savard,${ }^{163, \mathrm{e}}$ R. Sawada, ${ }^{160}$ C. Sawyer, ${ }^{140}$ L. Sawyer,${ }^{93}$ I. Sayago Galvan, ${ }^{170}$ C. Sbarra, ${ }^{21 b}$ A. Sbrizzi, ${ }^{64 a, 64 c}$ T. Scanlon, ${ }^{92}$ J. Schaarschmidt, ${ }^{145}$ P. Schacht,${ }^{12}$ D. Schaefer,,${ }^{35}$ L. Schaefer, ${ }^{133}$ U. Schäfer, ${ }^{97}$ A. C. Schaffer, ${ }^{62}$ D. Schaile, ${ }^{111}$ R. D. Schamberger, ${ }^{152}$ E. Schanet, ${ }^{111}$ C. Scharf, ${ }^{17}$ N. Scharmberg, ${ }^{98}$ V. A. Schegelsky, ${ }^{134}$ D. Scheirich, ${ }^{139}$ F. Schenck, ${ }^{17}$ M. Schernau, ${ }^{167}$ C. Schiavi, ${ }^{53 b, 53 a}$ L. K. Schildgen, ${ }^{22}$ Z. M. Schillaci, ${ }^{24}$ E. J. Schioppa,${ }^{65 a, 65 b}$ M. Schioppa, ${ }^{39 b, 39 a}$ B. Schlag, ${ }^{97}$ K. E. Schleicher, ${ }^{50}$ S. Schlenker, ${ }^{34}$ K. Schmieden, ${ }^{97}$ C. Schmitt, ${ }^{97}$ S. Schmitt, ${ }^{44}$ L. Schoeffel, ${ }^{141}$ A. Schoening, ${ }^{59 b}$ P. G. Scholer, ${ }^{50}$ E. Schopf, ${ }^{131}$ M. Schott, ${ }^{97}$ J. Schovancova, ${ }^{34}$ S. Schramm, ${ }^{52}$ F. Schroeder, ${ }^{178}$ H-C. Schultz-Coulon, ${ }^{59 a}$ M. Schumacher, ${ }^{50}$ B. A. Schumm, ${ }^{142}$ Ph. Schune, ${ }^{141}$ A. Schwartzman, ${ }^{150}$ T. A. Schwarz, ${ }^{103} \mathrm{Ph}$. Schwemling, ${ }^{141}$ R. Schwienhorst, ${ }^{104}$ A. Sciandra, ${ }^{142}$ G. Sciolla,${ }^{24}$ F. Scuri, ${ }^{69 a}$ F. Scutti, ${ }^{102}$ C. D. Sebastiani, ${ }^{88}$ K. Sedlaczek, ${ }^{45}$ P. Seema, ${ }^{17}$ S. C. Seidel, ${ }^{115}$ A. Seiden, ${ }^{142}$ B. D. Seidlitz, ${ }^{27}$ T. Seiss, ${ }^{35}$ C. Seitz, ${ }^{44}$ J. M. Seixas, ${ }^{78 b}$ G. Sekhniaidze, ${ }^{67 a}$ S. J. Sekula, ${ }^{40}$ L. P. Selem, ${ }^{4}$ N. Semprini-Cesari, ${ }^{21 b, 21 a}$ S. Sen, ${ }^{47}$ C. Serfon, ${ }^{27}$ L. Serin, ${ }^{62}$ L. Serkin, ${ }^{64 a, 64 b}$ M. Sessa, ${ }^{58 a}$ H. Severini, ${ }^{125}$ S. Sevova, ${ }^{150}$ F. Sforza ${ }^{53 b, 53 a}$ A. Sfyrla, ${ }^{52}$ E. Shabalina, ${ }^{51}$ R. Shaheen, ${ }^{151}$ J. D. Shahinian, ${ }^{133}$ N. W. Shaikh, ${ }^{43 a, 43 b}$ D. Shaked Renous ${ }^{176}$ L. Y. Shan, ${ }^{13 a}$ M. Shapiro, ${ }^{16}$ A. Sharma ${ }^{34}$ A. S. Sharma, ${ }^{1}$ S. Sharma, ${ }^{44}$ P. B. Shatalov, ${ }^{121}$ K. Shaw, ${ }^{153}$ S. M. Shaw,${ }^{98}$ P. Sherwood, ${ }^{92}$ L. Shi, ${ }^{92}$ C. O. Shimmin,${ }^{179}$ Y. Shimogama, ${ }^{175}$ M. Shimojima, ${ }^{113}$ J. D. Shinner, ${ }^{91}$

I. P. J. Shipsey, ${ }^{131}$ S. Shirabe,${ }^{52}$ M. Shiyakova, ${ }^{77}$ J. Shlomi,${ }^{176}$ M. J. Shochet,${ }^{35}$ J. Shojaii, ${ }^{102}$ D. R. Shope, ${ }^{151}$ S. Shrestha, ${ }^{124}$ E. M. Shrif,${ }^{31 f}$ M. J. Shroff, ${ }^{172}$ E. Shulga ${ }^{176}$ P. Sicho, ${ }^{137}$ A. M. Sickles, ${ }^{169}$ E. Sideras Haddad,${ }^{31 f}$ O. Sidiropoulou, ${ }^{34}$ A. Sidoti, ${ }^{21 b, 21 \mathrm{a}}$ F. Siegert, ${ }^{46}$ Dj. Sijacki, ${ }^{14}$ M. V. Silva Oliveira, ${ }^{34}$ S. B. Silverstein, ${ }^{43 a}$ S. Simion, ${ }^{62}$ R. Simoniello, ${ }^{34}$ S. Simsek, ${ }^{11 \mathrm{~b}}$ P. Sinervo, ${ }^{163}$ V. Sinetckii, ${ }^{110}$ S. Singh, ${ }^{149}$ S. Sinha, ${ }^{44}$ S. Sinha, ${ }^{31 \mathrm{f}}$ M. Sioli, ${ }^{21 \mathrm{~b}, 21 \mathrm{a}}$ I. Siral, ${ }^{128}$ 
S. Yu. Sivoklokov, ${ }^{110}$ J. Sjölin, ${ }^{43 a, 43 b}$ A. Skaf, ${ }^{51}$ E. Skorda, ${ }^{94}$ P. Skubic, ${ }^{125}$ M. Slawinska, ${ }^{82}$ K. Sliwa, ${ }^{166}$ V. Smakhtin, ${ }^{176}$ B. H. Smart, ${ }^{140}$ J. Smiesko, ${ }^{139}$ S. Yu. Smirnov, ${ }^{109}$ Y. Smirnov, ${ }^{109}$ L. N. Smirnova, ${ }^{110, \text { hh }}$ O. Smirnova, ${ }^{94}$ E. A. Smith, ${ }^{35}$ H. A. Smith, ${ }^{131}$ M. Smizanska, ${ }^{87}$ K. Smolek, ${ }^{138}$ A. Smykiewicz, ${ }^{82}$ A. A. Snesarev, ${ }^{108}$ H. L. Snoek, ${ }^{117}$ S. Snyder, ${ }^{27}$ R. Sobie, ${ }^{172, \mathrm{~m}}$ A. Soffer, ${ }^{158}$ F. Sohns,${ }^{51}$ C. A. Solans Sanchez,${ }^{34}$ E. Yu. Soldatov, ${ }^{109}$ U. Soldevila, ${ }^{170}$ A. A. Solodkov, ${ }^{120}$ S. Solomon, ${ }^{50}$ A. Soloshenko, ${ }^{77}$ O. V. Solovyanov, ${ }^{120}$ V. Solovyev, ${ }^{134}$ P. Sommer, ${ }^{146}$ H. Son, ${ }^{166}$ A. Sonay, ${ }^{12}$ W. Y. Song, ${ }^{164 b}$ A. Sopczak, ${ }^{138}$ A. L. Sopio, ${ }^{92}$ F. Sopkova, ${ }^{26 \mathrm{~b}}$ S. Sottocornola, ${ }^{68 \mathrm{a}, 68 \mathrm{~b}}$ R. Soualah, ${ }^{64 \mathrm{a}, 64 \mathrm{c}}$ A. M. Soukharev, ${ }^{119 b, 119 \mathrm{a}}$ Z. Soumaimi, ${ }^{33 \mathrm{e}}$ D. South, ${ }^{44}$ S. Spagnolo, ${ }^{65 a, 65 b}$ M. Spalla, ${ }^{112}$ M. Spangenberg, ${ }^{174}$ F. Spanò,${ }^{91}$ D. Sperlich,,${ }^{50}$ T. M. Spieker, ${ }^{59 a}$ G. Spigo, ${ }^{34}$ M. Spina, ${ }^{153}$ D. P. Spiteri, ${ }^{55}$ M. Spousta, ${ }^{139}$ A. Stabile,${ }^{66,66 b}$ B. L. Stamas, ${ }^{118}$ R. Stamen, ${ }^{59 a}$ M. Stamenkovic, ${ }^{117}$ A. Stampekis, ${ }^{19}$ M. Standke, ${ }^{22}$ E. Stanecka, ${ }^{82}$ B. Stanislaus, ${ }^{34}$ M. M. Stanitzki, ${ }^{44}$ M. Stankaityte, ${ }^{131}$ B. Stapf,${ }^{44}$ E. A. Starchenko, ${ }^{120}$ G. H. Stark, ${ }^{142}$ J. Stark, ${ }^{99}$ D. M. Starko, ${ }^{164 b}$ P. Staroba,${ }^{138}$ P. Starovoitov, ${ }^{59 a}$ S. Stärz, ${ }^{101}$ R. Staszewski, ${ }^{82}$ G. Stavropoulos, ${ }^{42}$ P. Steinberg, ${ }^{27}$ A. L. Steinhebel, ${ }^{128}$ B. Stelzer, ${ }^{149,164 a}$ H. J. Stelzer, ${ }^{135}$ O. Stelzer-Chilton, ${ }^{164 a}$ H. Stenzel, ${ }^{54}$ T. J. Stevenson, ${ }^{153}$ G. A. Stewart, ${ }^{34}$ M. C. Stockton ${ }^{34}$ G. Stoicea, ${ }^{25 b}$ M. Stolarski, ${ }^{136 a}$ S. Stonjek, ${ }^{112}$ A. Straessner, ${ }^{46}$ J. Strandberg, ${ }^{151}$ S. Strandberg, ${ }^{43 a, 43 b}$ M. Strauss, ${ }^{125}$ T. Strebler, ${ }^{99}$ P. Strizenec, ${ }^{26 b}$ R. Ströhmer, ${ }^{173}$ D. M. Strom, ${ }^{128}$ L. R. Strom, ${ }^{44}$ R. Stroynowski, ${ }^{40}$ A. Strubig, ${ }^{43 a, 43 b}$ S. A. Stucci, ${ }^{27}$ B. Stugu, ${ }^{15}$ J. Stupak, ${ }^{125}$ N. A. Styles, ${ }^{44}$ D. Su, ${ }^{150}$ S. Su, ${ }^{58 a}$ W. Su, ${ }^{58 d, 146,58 c}$ X. Su, ${ }^{58 \mathrm{a}}$ N. B. Suarez, ${ }^{135}$ K. Sugizaki, ${ }^{160}$ V. V. Sulin, ${ }^{108}$ M. J. Sullivan, ${ }^{88}$ D. M. S. Sultan,${ }^{52}$ S. Sultansoy, ${ }^{3 \mathrm{c}}$ T. Sumida, ${ }^{83}$ S. Sun, ${ }^{103}$ S. Sun, ${ }^{177}$ X. Sun, ${ }^{98}$ O. Sunneborn Gudnadottir, ${ }^{168}$ C. J. E. Suster, ${ }^{154}$ M. R. Sutton, ${ }^{153}$ M. Svatos, ${ }^{137}$ M. Swiatlowski, ${ }^{164 a}$ T. Swirski, ${ }^{173}$ I. Sykora, ${ }^{26 a}$ M. Sykora ${ }^{139}$ T. Sykora, ${ }^{139}$ D. Ta, ${ }^{97}$ K. Tackmann, ${ }^{44, i i}$ A. Taffard,${ }^{167}$ R. Tafirout, ${ }^{164 a}$ E. Tagiev, ${ }^{120}$ R. H. M. Taibah,${ }^{132}$ R. Takashima, ${ }^{84}$ K. Takeda, ${ }^{80}$ T. Takeshita,${ }^{147}$ E. P. Takeva,${ }^{48}$ Y. Takubo,${ }^{79}$ M. Talby, ${ }^{99}$ A. A. Talyshev, ${ }^{119 b, 119 a}$ K. C. Tam, ${ }^{60 b}$ N. M. Tamir, ${ }^{158}$ A. Tanaka, ${ }^{160}$ J. Tanaka, ${ }^{160}$ R. Tanaka, ${ }^{62}$ Z. Tao, ${ }^{171}$

S. Tapia Araya, ${ }^{76}$ S. Tapprogge, ${ }^{97}$ A. Tarek Abouelfadl Mohamed, ${ }^{104}$ S. Tarem,${ }^{157}$ K. Tariq ${ }^{58 b}$ G. Tarna, ${ }^{25 b, j j}$ G. F. Tartarelli, ${ }^{66 \mathrm{a}}$ P. Tas, ${ }^{139}$ M. Tasevsky, ${ }^{137}$ E. Tassi, ${ }^{39 b, 39 a}$ G. Tateno, ${ }^{160}$ Y. Tayalati, ${ }^{33 \mathrm{e}}$ G. N. Taylor, ${ }^{102}$ W. Taylor, ${ }^{164 \mathrm{~b}}$ H. Teagle ${ }^{88}$ A. S. Tee,${ }^{87}$ R. Teixeira De Lima, ${ }^{150}$ P. Teixeira-Dias, ${ }^{91}$ H. Ten Kate,${ }^{34}$ J. J. Teoh, ${ }^{117}$ K. Terashi, ${ }^{160}$ J. Terron, ${ }^{96}$ S. Terzo, ${ }^{12}$ M. Testa, ${ }^{49}$ R. J. Teuscher ${ }^{163, \mathrm{~m}}$ N. Themistokleous, ${ }^{48}$ T. Theveneaux-Pelzer, ${ }^{17}$ D. W. Thomas, ${ }^{91}$ J. P. Thomas, ${ }^{19}$ E. A. Thompson, ${ }^{44}$ P. D. Thompson, ${ }^{19}$ E. Thomson, ${ }^{133}$ E. J. Thorpe, ${ }^{90}$ Y. Tian, ${ }^{51}$ V. O. Tikhomirov, ${ }^{108, k k}$ Yu. A. Tikhonov, ${ }^{119 b, 119 a}$ S. Timoshenko, ${ }^{109}$ P. Tipton, ${ }^{179}$ S. Tisserant, ${ }^{99}$ S. H. Tlou, ${ }^{31 \mathrm{f}}$ A. Tnourji, ${ }^{36}$ K. Todome, ${ }^{21 b, 21 a}$ S. Todorova-Nova, ${ }^{139} \mathrm{~S}$. Todt, ${ }^{46} \mathrm{M}$. Togawa, ${ }^{79} \mathrm{~J}$. Tojo, ${ }^{85} \mathrm{~S}$. Tokár, ${ }^{26 a} \mathrm{~K}$. Tokushuku, ${ }^{79}$ E. Tolley, ${ }^{124} \mathrm{R}$. Tombs,${ }^{30}$ M. Tomoto, ${ }^{79,114}$ L. Tompkins, ${ }^{150}$ P. Tornambe,${ }^{100}$ E. Torrence, ${ }^{128}$ H. Torres,${ }^{46}$ E. Torró Pastor, ${ }^{170}$ M. Toscani, ${ }^{28}$ C. Tosciri, ${ }^{35}$ J. Toth,${ }^{99,11}$ D. R. Tovey, ${ }^{146}$ A. Traeet, ${ }^{15}$ C. J. Treado, ${ }^{122}$ T. Trefzger,,${ }^{173}$ A. Tricoli, ${ }^{27}$ I. M. Trigger, ${ }^{164 a}$ S. Trincaz-Duvoid, ${ }^{132}$ D. A. Trischuk, ${ }^{171} \mathrm{~W}$. Trischuk, ${ }^{163} \mathrm{~B}$. Trocmé, ${ }^{56}$ A. Trofymov, ${ }^{62} \mathrm{C}$. Troncon, ${ }^{66 \mathrm{a}} \mathrm{F}$. Trovato, ${ }^{153} \mathrm{~L}$. Truong, ${ }^{31 \mathrm{c}} \mathrm{M}$. Trzebinski ${ }^{82}$ A. Trzupek, ${ }^{82}$ F. Tsai, ${ }^{152}$ A. Tsiamis, ${ }^{159}$ P. V. Tsiareshka, ${ }^{105, b b}$ A. Tsirigotis, ${ }^{159, c c}$ V. Tsiskaridze,${ }^{152}$ E. G. Tskhadadze, ${ }^{156 a}$ M. Tsopoulou, ${ }^{159}$ I. I. Tsukerman, ${ }^{121}$ V. Tsulaia, ${ }^{16}$ S. Tsuno,${ }^{79}$ O. Tsur, ${ }^{157}$ D. Tsybychev, ${ }^{152}$ Y. Tu, ${ }^{60 b}$ A. Tudorache, ${ }^{25 b}$ V. Tudorache, ${ }^{25 b}$ A. N. Tuna,${ }^{34}$ S. Turchikhin,${ }^{77}$ D. Turgeman, ${ }^{176}$ I. Turk Cakir, ${ }^{3 b, m m}$ R. J. Turner, ${ }^{19}$ R. Turra, ${ }^{66 a}$ P. M. Tuts, ${ }^{37}$ S. Tzamarias, ${ }^{159}$ P. Tzanis, ${ }^{9}$ E. Tzovara,${ }^{97}$ K. Uchida, ${ }^{160}$ F. Ukegawa, ${ }^{165}$ G. Unal,${ }^{34}$ M. Unal, ${ }^{10}$ A. Undrus,${ }^{27}$ G. Unel,,${ }^{167}$ F. C. Ungaro, ${ }^{102}$ K. Uno, ${ }^{160}$ J. Urban, ${ }^{26 b}$ P. Urquijo, ${ }^{102}$ G. Usai, ${ }^{7}$ R. Ushioda, ${ }^{161}$ M. Usman, ${ }^{107}$ Z. Uysal, ${ }^{11 d}$ V. Vacek, ${ }^{138}$ B. Vachon, ${ }^{101}$ K. O. H. Vadla, ${ }^{130}$ T. Vafeiadis, ${ }^{34}$ C. Valderanis, ${ }^{111}$ E. Valdes Santurio, ${ }^{43 a, 43 b}$ M. Valente,${ }^{164 a}$ S. Valentinetti, ${ }^{21 \mathrm{~b}, 21 \mathrm{a}}$ A. Valero, ${ }^{170}$ L. Valéry, ${ }^{44}$ R. A. Vallance, ${ }^{19}$ A. Vallier, ${ }^{99}$ J. A. Valls Ferrer, ${ }^{170}$ T. R. Van Daalen, ${ }^{12}$ P. Van Gemmeren, ${ }^{5}$ S. Van Stroud,${ }^{92}$ I. Van Vulpen, ${ }^{117}$ M. Vanadia, ${ }^{71 a, 71 b}$ W. Vandelli, ${ }^{34}$ M. Vandenbroucke, ${ }^{141}$

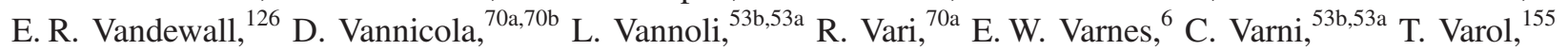
D. Varouchas, ${ }^{62}$ K. E. Varvell, ${ }^{154}$ M. E. Vasile, ${ }^{25 b}$ L. Vaslin, ${ }^{36}$ G. A. Vasquez, ${ }^{172}$ F. Vazeille, ${ }^{36}$ D. Vazquez Furelos,${ }^{12}$ T. Vazquez Schroeder, ${ }^{34}$ J. Veatch, ${ }^{51}$ V. Vecchio, ${ }^{98}$ M. J. Veen, ${ }^{117}$ I. Veliscek, ${ }^{131}$ L. M. Veloce,${ }^{163}$ F. Veloso, ${ }^{136 a, 136 c}$ S. Veneziano, ${ }^{70 a}$ A. Ventura, ${ }^{65 a, 65 b}$ A. Verbytskyi, ${ }^{112}$ M. Verducci, ${ }^{69 a, 69 b}$ C. Vergis,${ }^{22}$ M. Verissimo De Araujo, ${ }^{78 b}$ W. Verkerke, ${ }^{117}$ A. T. Vermeulen, ${ }^{117}$ J. C. Vermeulen, ${ }^{117}$ C. Vernieri, ${ }^{150}$ P. J. Verschuuren, ${ }^{91}$ M. L. Vesterbacka, ${ }^{122}$ M. C. Vetterli, ${ }^{149, e}$ N. Viaux Maira, ${ }^{143 e}$ T. Vickey, ${ }^{146}$ O. E. Vickey Boeriu, ${ }^{146}$ G. H. A. Viehhauser, ${ }^{131}$ L. Vigani, ${ }^{59 b}$ M. Villa, ${ }^{21 b, 21 a}$ M. Villaplana Perez, ${ }^{170}$ E. M. Villhauer, ${ }^{48}$ E. Vilucchi, ${ }^{49}$ M. G. Vincter ${ }^{32}$ G. S. Virdee, ${ }^{19}$ A. Vishwakarma, ${ }^{48}$ C. Vittori, ${ }^{21 b, 21 a}$ I. Vivarelli, ${ }^{153}$ V. Vladimirov, ${ }^{174}$ E. Voevodina, ${ }^{112}$ M. Vogel, ${ }^{178}$ P. Vokac, ${ }^{138}$ J. Von Ahnen, ${ }^{44}$ S. E. von Buddenbrock, ${ }^{31 f}$ E. Von Toerne, ${ }^{22}$ V. Vorobel, ${ }^{139}$ K. Vorobev, ${ }^{109}$ M. Vos,${ }^{170}$ J. H. Vossebeld, ${ }^{88}$ M. Vozak, ${ }^{98}$ N. Vranjes, ${ }^{14}$ M. Vranjes Milosavljevic, ${ }^{14}$ V. Vrba, ${ }^{138, a}$ M. Vreeswijk, ${ }^{117}$ N. K. Vu, ${ }^{99}$ R. Vuillermet, ${ }^{34}$ I. Vukotic, ${ }^{35}$ S. Wada ${ }^{165}$ C. Wagner, ${ }^{100}$ P. Wagner, ${ }^{22}$ W. Wagner, ${ }^{178}$ S. Wahdan, ${ }^{178}$ H. Wahlberg, ${ }^{86}$ R. Wakasa, ${ }^{165}$ M. Wakida, ${ }^{114}$ V. M. Walbrecht, ${ }^{112}$ J. Walder, ${ }^{140}$ R. Walker, ${ }^{111}$ S. D. Walker, ${ }^{91}$ W. Walkowiak, ${ }^{148}$ A. M. Wang, ${ }^{57}$ A. Z. Wang, ${ }^{178}$ C. Wang, ${ }^{58 a}$ 
C. Wang, ${ }^{58 \mathrm{c}}$ H. Wang, ${ }^{16}$ J. Wang, ${ }^{60 \mathrm{a}}$ P. Wang, ${ }^{40}$ R.-J. Wang, ${ }^{97}$ R. Wang, ${ }^{57}$ R. Wang, ${ }^{118}$ S. M. Wang, ${ }^{155}$ S. Wang, ${ }^{58 b}$ T. Wang, ${ }^{58 \mathrm{a}}$ W. T. Wang, ${ }^{58 \mathrm{a}}$ W. X. Wang, ${ }^{58 \mathrm{a}}$ X. Wang, ${ }^{169}$ Y. Wang, ${ }^{58 \mathrm{a}}$ Z. Wang, ${ }^{103}$ C. Wanotayaroj, ${ }^{34}$ A. Warburton, ${ }^{101}$ C. P. Ward,${ }^{30}$ R. J. Ward ${ }^{19}$ N. Warrack ${ }^{55}$ A. T. Watson, ${ }^{19}$ M. F. Watson, ${ }^{19}$ G. Watts, ${ }^{145}$ B. M. Waugh,${ }^{92}$ A. F. Webb, ${ }^{10}$ C. Weber ${ }^{27}$ M. S. Weber, ${ }^{18}$ S. A. Weber, ${ }^{32}$ S. M. Weber, ${ }^{59 a}$ C. Wei, ${ }^{58 a}$ Y. Wei ${ }^{131}$ A. R. Weidberg, ${ }^{131}$ J. Weingarten, ${ }^{45}$ M. Weirich,${ }^{97}$ C. Weiser, ${ }^{50}$ T. Wenaus,${ }^{27}$ B. Wendland, ${ }^{45}$ T. Wengler, ${ }^{34}$ S. Wenig, ${ }^{34}$ N. Wermes,${ }^{22}$ M. Wessels, ${ }^{59 a}$ K. Whalen, ${ }^{128}$ A. M. Wharton, ${ }^{87}$ A. S. White, ${ }^{57}$ A. White, ${ }^{7}$ M. J. White, ${ }^{1}$ D. Whiteson, ${ }^{167}$ W. Wiedenmann, ${ }^{177}$ C. Wiel,${ }^{46}$ M. Wielers,${ }^{140}$ N. Wieseotte, ${ }^{97}$ C. Wiglesworth, ${ }^{38}$ L. A. M. Wiik-Fuchs,${ }^{50}$ D. J. Wilbern, ${ }^{125}$ H. G. Wilkens, ${ }^{34}$ L. J. Wilkins, ${ }^{91}$

D. M. Williams, ${ }^{37}$ H. H. Williams, ${ }^{133}$ S. Williams, ${ }^{30}$ S. Willocq, ${ }^{100}$ P. J. Windischhofer, ${ }^{131}$ I. Wingerter-Seez, ${ }^{4}$

F. Winklmeier, ${ }^{128}$ B. T. Winter, ${ }^{50}$ M. Wittgen, ${ }^{150}$ M. Wobisch, ${ }^{93}$ A. Wolf,${ }^{97}$ R. Wölker, ${ }^{131}$ J. Wollrath, ${ }^{167}$ M. W. Wolter, ${ }^{82}$ H. Wolters, ${ }^{136 a, 136 c}$ V. W. S. Wong, ${ }^{172}$ A. F. Wongel, ${ }^{44}$ S. D. Worm, ${ }^{44}$ B. K. Wosiek, ${ }^{82}$ K. W. Woźniak, ${ }^{82}$ K. Wraight, ${ }^{55}$ J. Wu, ${ }^{13 a, 13 d}$ S. L. Wu, ${ }^{177}$ X. Wu ${ }^{52}$ Y. Wu, ${ }^{58 a}$ Z. Wu, ${ }^{141,58 a}$ J. Wuerzinger, ${ }^{131}$ T. R. Wyatt, ${ }^{98}$ B. M. Wynne, ${ }^{48}$ S. Xella, ${ }^{38}$ J. Xiang, ${ }^{60 c}$ X. Xiao, ${ }^{103}$ X. Xie, ${ }^{58 a}$ I. Xiotidis, ${ }^{153}$ D. Xu, ${ }^{13 a}$ H. Xu ${ }^{58 a}$ H. Xu, ${ }^{58 a}$ L. Xu ${ }^{58 a}$ R. Xu, ${ }^{133}$ W. Xu, ${ }^{103}$ Y. Xu, ${ }^{13 b}$ Z. Xu ${ }^{58 b}$ Z. Xu ${ }^{150}$ B. Yabsley, ${ }^{154}$ S. Yacoob,${ }^{31 a}$ N. Yamaguchi, ${ }^{85}$ Y. Yamaguchi, ${ }^{161}$ M. Yamatani, ${ }^{160}$ H. Yamauchi, ${ }^{165}$ T. Yamazaki, ${ }^{16}$ Y. Yamazaki, ${ }^{80}$ J. Yan, ${ }^{58 c}$ Z. Yan, ${ }^{23}$ H. J. Yang, ${ }^{58 c, 58 d}$ H. T. Yang, ${ }^{16}$ S. Yang, ${ }^{58 a}$ T. Yang, ${ }^{60 c}$ X. Yang, ${ }^{58 a}$ X. Yang, ${ }^{13 a}$ Y. Yang, ${ }^{160}$ Z. Yang, ${ }^{103,58 a}$ W-M. Yao, ${ }^{16}$ Y. C. Yap, ${ }^{44}$ H. Ye, ${ }^{13 \mathrm{c}}$ J. Ye,${ }^{40}$ S. Ye, ${ }^{27}$ I. Yeletskikh, ${ }^{77}$ M. R. Yexley,${ }^{87}$ P. Yin,${ }^{37}$ K. Yorita, ${ }^{175}$ K. Yoshihara,${ }^{76}$ C. J. S. Young, ${ }^{34}$ C. Young, ${ }^{150}$ R. Yuan,${ }^{58, n n}$ X. Yue, ${ }^{59 a}$ M. Zaazoua,${ }^{33 e}$ B. Zabinski, ${ }^{82}$ G. Zacharis, ${ }^{9}$ E. Zaffaroni, ${ }^{52}$ J. Zahreddine, ${ }^{99}$ A. M. Zaitsev, ${ }^{120, \mathrm{i}}$ T. Zakareishvili, ${ }^{156 \mathrm{~b}}$ N. Zakharchuk, ${ }^{32}$ S. Zambito, ${ }^{34}$ D. Zanzi ${ }^{50}$ S. V. Zeißner, ${ }^{45}$ C. Zeitnitz, ${ }^{178}$ G. Zemaityte, ${ }^{131}$ J. C. Zeng, ${ }^{169}$ O. Zenin, ${ }^{120}$ T. Ženiš ${ }^{26 a}$ S. Zenz, ${ }^{90}$ S. Zerradi, ${ }^{33 a}$ D. Zerwas, ${ }^{62}$ M. Zgubič, ${ }^{131}$ B. Zhang, ${ }^{13 \mathrm{c}}$ D. F. Zhang, ${ }^{13 \mathrm{~b}}$ G. Zhang, ${ }^{13 \mathrm{~b}}$ J. Zhang, ${ }^{5}$ K. Zhang, ${ }^{13 \mathrm{a}}$ L. Zhang, ${ }^{13 \mathrm{c}}$ M. Zhang, ${ }^{169}$ R. Zhang, ${ }^{177}$ S. Zhang, ${ }^{103}$ X. Zhang, ${ }^{58 \mathrm{c}}$ X. Zhang, ${ }^{58 \mathrm{~b}}$ Z. Zhang, ${ }^{62}$ P. Zhao, ${ }^{47}$ Y. Zhao, ${ }^{142}$ Z. Zhao, ${ }^{58 \mathrm{a}}$ A. Zhemchugov, ${ }^{77}$ Z. Zheng, ${ }^{103}$ D. Zhong, ${ }^{169}$ B. Zhou, ${ }^{103}$ C. Zhou, ${ }^{177}$ H. Zhou, ${ }^{6}$ N. Zhou, ${ }^{58 c}$ Y. Zhou, ${ }^{6}$ C. G. Zhu, ${ }^{58 b}$ C. Zhu, ${ }^{13 a, 13 d}$ H. L. Zhu, ${ }^{58 a}$ H. Zhu, ${ }^{13 a}$ J. Zhu, ${ }^{103}$ Y. Zhu, ${ }^{58 a}$ X. Zhuang, ${ }^{13 a}$ K. Zhukov, ${ }^{108}$ V. Zhulanov, ${ }^{19 b, 119 a}$ D. Zieminska, ${ }^{63}$ N. I. Zimine, ${ }^{77}$ S. Zimmermann, ${ }^{50, a}$ M. Ziolkowski, ${ }^{148}$ L. Živkovic,,${ }^{14}$ A. Zoccoli, ${ }^{21 b, 21 a}$ K. Zoch, ${ }^{52}$ T. G. Zorbas, ${ }^{146}$ O. Zormpa, ${ }^{42}$ W. Zou, ${ }^{37}$ and L. Zwalinski ${ }^{34}$

\title{
(ATLAS Collaboration)
}

\author{
${ }^{1}$ Department of Physics, University of Adelaide, Adelaide, Australia \\ ${ }^{2}$ Department of Physics, University of Alberta, Edmonton AB, Canada \\ ${ }^{3 a}$ Department of Physics, Ankara University, Ankara, Turkey \\ ${ }^{3 \mathrm{~b}}$ Istanbul Aydin University, Application and Research Center for Advanced Studies, Istanbul, Turkey \\ ${ }^{3 c}$ Division of Physics, TOBB University of Economics and Technology, Ankara, Turkey \\ ${ }^{4}$ LAPP, Univ. Savoie Mont Blanc, CNRS/IN2P3, Annecy, France \\ ${ }^{5}$ High Energy Physics Division, Argonne National Laboratory, Argonne Illinois, United States of America \\ ${ }^{6}$ Department of Physics, University of Arizona, Tucson Arizona, USA \\ ${ }^{7}$ Department of Physics, University of Texas at Arlington, Arlington Texas, USA \\ ${ }^{8}$ Physics Department, National and Kapodistrian University of Athens, Athens, Greece \\ ${ }^{9}$ Physics Department, National Technical University of Athens, Zografou, Greece \\ ${ }^{10}$ Department of Physics, University of Texas at Austin, Austin Texas, USA \\ ${ }^{11 a}$ Bahcesehir University, Faculty of Engineering and Natural Sciences, Istanbul, Turkey \\ ${ }^{11 \mathrm{~b}}$ Istanbul Bilgi University, Faculty of Engineering and Natural Sciences, Istanbul, Turkey \\ ${ }^{11 \mathrm{c}}$ Department of Physics, Bogazici University, Istanbul, Turkey \\ ${ }^{11 \mathrm{~d}}$ Department of Physics Engineering, Gaziantep University, Gaziantep, Turkey \\ ${ }^{12}$ Institut de Física d'Altes Energies (IFAE), Barcelona Institute of Science and Technology, \\ Barcelona, Spain \\ ${ }^{13 a}$ Institute of High Energy Physics, Chinese Academy of Sciences, Beijing, China \\ ${ }^{13 \mathrm{~b}}$ Physics Department, Tsinghua University, Beijing, China \\ ${ }^{13 c}$ Department of Physics, Nanjing University, Nanjing, China \\ ${ }^{13 \mathrm{~d}}$ University of Chinese Academy of Science (UCAS), Beijing, China \\ ${ }^{14}$ Institute of Physics, University of Belgrade, Belgrade, Serbia \\ ${ }^{15}$ Department for Physics and Technology, University of Bergen, Bergen, Norway \\ ${ }^{16}$ Physics Division, Lawrence Berkeley National Laboratory and University of California, \\ Berkeley California, USA \\ ${ }^{17}$ Institut für Physik, Humboldt Universität zu Berlin, Berlin, Germany
}


${ }^{18}$ Albert Einstein Center for Fundamental Physics and Laboratory for High Energy Physics, University of Bern, Bern, Switzerland

${ }^{19}$ School of Physics and Astronomy, University of Birmingham, Birmingham, United Kingdom

${ }^{20 \mathrm{a}}$ Facultad de Ciencias y Centro de Investigaciónes, Universidad Antonio Nariño, Bogotá, Colombia

${ }^{20 \mathrm{~b}}$ Departamento de Física, Universidad Nacional de Colombia, Bogotá, Colombia, Colombia

${ }^{21 \mathrm{a}}$ INFN Bologna and Universita' di Bologna, Dipartimento di Fisica, Italy

${ }^{21 \mathrm{~b}}$ INFN Sezione di Bologna, Italy

${ }^{22}$ Physikalisches Institut, Universität Bonn, Bonn, Germany

${ }^{23}$ Department of Physics, Boston University, Boston Massachusetts, USA

${ }^{24}$ Department of Physics, Brandeis University, Waltham Massachusetts, USA

${ }^{25 a}$ Transilvania University of Brasov, Brasov, Romania

${ }^{25 \mathrm{~b}}$ Horia Hulubei National Institute of Physics and Nuclear Engineering, Bucharest, Romania

${ }^{25 \mathrm{c}}$ Department of Physics, Alexandru Ioan Cuza University of Iasi, Iasi, Romania

${ }^{25 \mathrm{~d}}$ National Institute for Research and Development of Isotopic and Molecular Technologies, Physics Department, Cluj-Napoca, Romania

${ }^{25 e}$ University Politehnica Bucharest, Bucharest, Romania

${ }^{25 \mathrm{f}}$ West University in Timisoara, Timisoara, Romania

${ }^{26 a}$ Faculty of Mathematics, Physics and Informatics, Comenius University, Bratislava, Slovak Republic

${ }^{26 \mathrm{~b}}$ Department of Subnuclear Physics, Institute of Experimental Physics of the Slovak Academy of Sciences, Kosice, Slovak Republic

${ }^{27}$ Physics Department, Brookhaven National Laboratory, Upton New York, USA

${ }^{28}$ Departamento de Física, Universidad de Buenos Aires, Buenos Aires, Argentina

${ }^{29}$ California State University, California, USA

${ }^{30}$ Cavendish Laboratory, University of Cambridge, Cambridge, United Kingdom

${ }^{31 \mathrm{a}}$ Department of Physics, University of Cape Town, Cape Town, South Africa

${ }^{31 \mathrm{~b}}$ iThemba Labs, Western Cape, South Africa

${ }^{31 \mathrm{c}}$ Department of Mechanical Engineering Science, University of Johannesburg, Johannesburg, South Africa

${ }^{31 \mathrm{~d}}$ National Institute of Physics, University of the Philippines Diliman, Philippines

${ }^{31 \mathrm{e}}$ University of South Africa, Department of Physics, Pretoria, South Africa

${ }^{31 \mathrm{f}}$ School of Physics, University of the Witwatersrand, Johannesburg, South Africa

${ }^{32}$ Department of Physics, Carleton University, Ottawa ON, Canada

${ }^{33 a}$ Faculté des Sciences Ain Chock, Réseau Universitaire de Physique des Hautes Energies-Université Hassan II, Casablanca, Morocco

${ }^{33 \mathrm{~b}}$ Faculté des Sciences, Université Ibn-Tofail, Kénitra, Morocco

${ }^{33 c}$ Faculté des Sciences Semlalia, Université Cadi Ayyad, LPHEA-Marrakech, Morocco

${ }^{33 \mathrm{~d}}$ LPMR, Faculté des Sciences, Université Mohamed Premier, Oujda, Morocco ${ }^{33 \mathrm{e}}$ Faculté des sciences, Université Mohammed V, Rabat, Morocco

${ }^{33 \mathrm{f}}$ Mohammed VI Polytechnic University, Ben Guerir, Morocco

${ }^{34}$ CERN, Geneva, Switzerland

${ }^{35}$ Enrico Fermi Institute, University of Chicago, Chicago Illinois, USA

${ }^{36}$ LPC, Université Clermont Auvergne, CNRS/IN2P3, Clermont-Ferrand, France

${ }^{37}$ Nevis Laboratory, Columbia University, Irvington New York, USA

${ }^{38}$ Niels Bohr Institute, University of Copenhagen, Copenhagen, Denmark

${ }^{39 a}$ Dipartimento di Fisica, Università della Calabria, Rende, Italy

${ }^{39 b}$ INFN Gruppo Collegato di Cosenza, Laboratori Nazionali di Frascati, Italy

${ }^{40}$ Physics Department, Southern Methodist University, Dallas Texas, USA

${ }^{41}$ Physics Department, University of Texas at Dallas, Richardson Texas, USA

${ }^{42}$ National Centre for Scientific Research "Demokritos", Agia Paraskevi, Greece

${ }^{43 a}$ Department of Physics, Stockholm University, Sweden

${ }^{43 \mathrm{~b}}$ Oskar Klein Centre, Stockholm, Sweden

${ }^{44}$ Deutsches Elektronen-Synchrotron DESY, Hamburg and Zeuthen, Germany

${ }^{45}$ Lehrstuhl für Experimentelle Physik IV, Technische Universität Dortmund, Dortmund, Germany

${ }^{46}$ Institut für Kern- und Teilchenphysik, Technische Universität Dresden, Dresden, Germany

${ }^{47}$ Department of Physics, Duke University, Durham North Carolina, USA

${ }^{48}$ SUPA-School of Physics and Astronomy, University of Edinburgh, Edinburgh, United Kingdom

${ }^{49}$ INFN e Laboratori Nazionali di Frascati, Frascati, Italy

${ }^{50}$ Physikalisches Institut, Albert-Ludwigs-Universität Freiburg, Freiburg, Germany

${ }^{51}$ II. Physikalisches Institut, Georg-August-Universität Göttingen, Göttingen, Germany

${ }^{52}$ Département de Physique Nucléaire et Corpusculaire, Université de Genève, Genève, Switzerland 
${ }^{53 a}$ Dipartimento di Fisica, Università di Genova, Genova, Italy

${ }^{53 \mathrm{~b}}$ INFN Sezione di Genova, Italy

${ }^{54}$ II. Physikalisches Institut, Justus-Liebig-Universität Giessen, Giessen, Germany

${ }^{55}$ SUPA-School of Physics and Astronomy, University of Glasgow, Glasgow, United Kingdom

${ }^{56}$ LPSC, Université Grenoble Alpes, CNRS/IN2P3, Grenoble INP, Grenoble, France

${ }^{57}$ Laboratory for Particle Physics and Cosmology, Harvard University, Cambridge Massachusetts, USA

${ }^{58 a}$ Department of Modern Physics and State Key Laboratory of Particle Detection and Electronics,

University of Science and Technology of China, Hefei, China

${ }^{58 \mathrm{~b}}$ Institute of Frontier and Interdisciplinary Science and Key Laboratory of Particle Physics and Particle Irradiation (MOE), Shandong University, Qingdao, China

${ }^{58 \mathrm{c}}$ School of Physics and Astronomy, Shanghai Jiao Tong University, Key Laboratory for Particle Astrophysics and Cosmology (MOE), SKLPPC, Shanghai, China

${ }^{58 \mathrm{~d}}$ Tsung-Dao Lee Institute, Shanghai, China

${ }^{59 a}$ Kirchhoff-Institut für Physik, Ruprecht-Karls-Universität Heidelberg, Heidelberg, Germany

${ }^{59 \mathrm{~b}}$ Physikalisches Institut, Ruprecht-Karls-Universität Heidelberg, Heidelberg, Germany

${ }^{60 \mathrm{a}}$ Department of Physics, Chinese University of Hong Kong, Shatin, N.T., Hong Kong, China

${ }^{60 \mathrm{~b}}$ Department of Physics, University of Hong Kong, Hong Kong, China

${ }^{60 \mathrm{c}}$ Department of Physics and Institute for Advanced Study, Hong Kong University of Science and

Technology, Clear Water Bay, Kowloon, Hong Kong, China

${ }^{61}$ Department of Physics, National Tsing Hua University, Hsinchu, Taiwan

${ }^{62}$ IJCLab, Université Paris-Saclay, CNRS/IN2P3, 91405, Orsay, France

${ }^{63}$ Department of Physics, Indiana University, Bloomington Indiana, USA

${ }^{64 a}$ INFN Gruppo Collegato di Udine, Sezione di Trieste, Udine, Italy

${ }^{64 \mathrm{~b}}$ ICTP, Trieste, Italy

${ }^{64 c}$ Dipartimento Politecnico di Ingegneria e Architettura, Università di Udine, Udine, Italy

${ }^{65 a}$ INFN Sezione di Lecce, Italy

${ }^{65 \mathrm{~b}}$ Dipartimento di Matematica e Fisica, Università del Salento, Lecce, Italy

${ }^{66 a}$ INFN Sezione di Milano, Italy

${ }^{66 \mathrm{~b}}$ Dipartimento di Fisica, Università di Milano, Milano, Italy

${ }^{67 a}$ INFN Sezione di Napoli, Italy

${ }^{67 \mathrm{~b}}$ Dipartimento di Fisica, Università di Napoli, Napoli, Italy

${ }^{68 \mathrm{a}}$ INFN Sezione di Pavia, Italy

${ }^{68 \mathrm{~b}}$ Dipartimento di Fisica, Università di Pavia, Pavia, Italy

${ }^{69 a}$ INFN Sezione di Pisa, Italy

${ }^{69 \mathrm{~b}}$ Dipartimento di Fisica E. Fermi, Università di Pisa, Pisa, Italy

${ }^{70 a}$ INFN Sezione di Roma, Italy

${ }^{70 \mathrm{~b}}$ Dipartimento di Fisica, Sapienza Università di Roma, Roma, Italy

${ }^{71 a}$ INFN Sezione di Roma Tor Vergata, Italy

${ }^{71 \mathrm{~b}}$ Dipartimento di Fisica, Università di Roma Tor Vergata, Roma, Italy

${ }^{72 \mathrm{a}}$ INFN Sezione di Roma Tre, Italy

${ }^{72 \mathrm{~b}}$ Dipartimento di Matematica e Fisica, Università Roma Tre, Roma, Italy

${ }^{73 a}$ INFN-TIFPA, Italy

${ }^{73 b}$ Università degli Studi di Trento, Trento, Italy

${ }^{74}$ Institut für Astro- und Teilchenphysik, Leopold-Franzens-Universität, Innsbruck, Austria

${ }^{75}$ University of Iowa, Iowa City Iowa, USA

${ }^{76}$ Department of Physics and Astronomy, Iowa State University, Ames Iowa, USA

${ }^{77}$ Joint Institute for Nuclear Research, Dubna, Russia

${ }^{78}$ Departamento de Engenharia Elétrica, Universidade Federal de Juiz de Fora (UFJF),

Juiz de Fora, Brazil

${ }^{78 b}$ Universidade Federal do Rio De Janeiro COPPE/EE/IF, Rio de Janeiro, Brazil

${ }^{78 c}$ Instituto de Física, Universidade de São Paulo, São Paulo, Brazil

${ }^{79}$ KEK, High Energy Accelerator Research Organization, Tsukuba, Japan

${ }^{80}$ Graduate School of Science, Kobe University, Kobe, Japan

${ }^{81 \mathrm{a} A G H}$ University of Science and Technology, Faculty of Physics and Applied Computer Science, Krakow, Poland

${ }^{81 \mathrm{~b}}$ Marian Smoluchowski Institute of Physics, Jagiellonian University, Krakow, Poland

${ }^{82}$ Institute of Nuclear Physics Polish Academy of Sciences, Krakow, Poland

${ }^{83}$ Faculty of Science, Kyoto University, Kyoto, Japan

${ }^{84}$ Kyoto University of Education, Kyoto, Japan 
${ }^{85}$ Research Center for Advanced Particle Physics and Department of Physics, Kyushu University, Fukuoka, Japan

${ }^{86}$ Instituto de Física La Plata, Universidad Nacional de La Plata and CONICET, La Plata, Argentina

${ }^{87}$ Physics Department, Lancaster University, Lancaster, United Kingdom

${ }^{88}$ Oliver Lodge Laboratory, University of Liverpool, Liverpool, United Kingdom

${ }^{89}$ Department of Experimental Particle Physics, Jožef Stefan Institute and Department of Physics, University of Ljubljana, Ljubljana, Slovenia

${ }^{90}$ School of Physics and Astronomy, Queen Mary University of London, London, United Kingdom

${ }^{91}$ Department of Physics, Royal Holloway University of London, Egham, United Kingdom

${ }^{92}$ Department of Physics and Astronomy, University College London, London, United Kingdom

${ }^{93}$ Louisiana Tech University, Ruston Louisiana, USA

${ }^{94}$ Fysiska institutionen, Lunds universitet, Lund, Sweden

${ }^{95}$ Centre de Calcul de l'Institut National de Physique Nucléaire et de Physique des Particules (IN2P3), Villeurbanne, France

${ }^{96}$ Departamento de Física Teorica C-15 and CIAFF, Universidad Autónoma de Madrid, Madrid, Spain

${ }^{97}$ Institut für Physik, Universität Mainz, Mainz, Germany

${ }^{98}$ School of Physics and Astronomy, University of Manchester, Manchester, United Kingdom

${ }^{99}$ CPPM, Aix-Marseille Université, CNRS/IN2P3, Marseille, France

${ }^{100}$ Department of Physics, University of Massachusetts, Amherst Massachusetts, USA

${ }^{101}$ Department of Physics, McGill University, Montreal QC, Canada

${ }^{102}$ School of Physics, University of Melbourne, Victoria, Australia

${ }^{103}$ Department of Physics, University of Michigan, Ann Arbor Michigan, USA

${ }^{104}$ Department of Physics and Astronomy, Michigan State University, East Lansing Michigan, USA

${ }^{105}$ B.I. Stepanov Institute of Physics, National Academy of Sciences of Belarus, Minsk, Belarus

${ }^{106}$ Research Institute for Nuclear Problems of Byelorussian State University, Minsk, Belarus

${ }^{107}$ Group of Particle Physics, University of Montreal, Montreal QC, Canada

${ }^{108}$ P.N. Lebedev Physical Institute of the Russian Academy of Sciences, Moscow, Russia

${ }^{109}$ National Research Nuclear University MEPhI, Moscow, Russia

${ }^{110}$ D.V. Skobeltsyn Institute of Nuclear Physics, M.V. Lomonosov Moscow State University,

Moscow, Russia

${ }^{111}$ Fakultät für Physik, Ludwig-Maximilians-Universität München, München, Germany

${ }^{112}$ Max-Planck-Institut für Physik (Werner-Heisenberg-Institut), München, Germany

${ }^{113}$ Nagasaki Institute of Applied Science, Nagasaki, Japan

${ }^{114}$ Graduate School of Science and Kobayashi-Maskawa Institute, Nagoya University, Nagoya, Japan

${ }^{115}$ Department of Physics and Astronomy, University of New Mexico, Albuquerque New Mexico, USA

${ }^{116}$ Institute for Mathematics, Astrophysics and Particle Physics, Radboud University/Nikhef, Nijmegen, Netherlands

${ }^{117}$ Nikhef National Institute for Subatomic Physics and University of Amsterdam, Amsterdam, Netherlands

${ }^{118}$ Department of Physics, Northern Illinois University, DeKalb Illinois, USA

${ }^{119 a}$ Budker Institute of Nuclear Physics and NSU, SB RAS, Novosibirsk, Russia

${ }^{119 b}$ Novosibirsk State University Novosibirsk, Russia

${ }^{120}$ Institute for High Energy Physics of the National Research Centre Kurchatov Institute, Protvino, Russia

${ }^{121}$ Institute for Theoretical and Experimental Physics named by A.I. Alikhanov of National Research

Centre "Kurchatov Institute", Moscow, Russia

${ }^{122}$ Department of Physics, New York University, New York New York, USA

${ }^{123}$ Ochanomizu University, Otsuka, Bunkyo-ku, Tokyo, Japan

${ }^{124}$ The Ohio State University, Columbus Ohio, USA

${ }^{125}$ Homer L. Dodge Department of Physics and Astronomy, University of Oklahoma, Norman Oklahoma, USA

${ }^{126}$ Department of Physics, Oklahoma State University, Stillwater Oklahoma, USA

${ }^{127}$ Palacký University, Joint Laboratory of Optics, Olomouc, Czech Republic

${ }^{128}$ Institute for Fundamental Science, University of Oregon, Eugene, Oregon, USA

${ }^{129}$ Graduate School of Science, Osaka University, Osaka, Japan

${ }^{130}$ Department of Physics, University of Oslo, Oslo, Norway

${ }^{131}$ Department of Physics, Oxford University, Oxford, United Kingdom

${ }^{132}$ LPNHE, Sorbonne Université, Université de Paris, CNRS/IN2P3, Paris, France

${ }^{133}$ Department of Physics, University of Pennsylvania, Philadelphia Pennsylvania, USA

${ }^{134}$ Konstantinov Nuclear Physics Institute of National Research Centre "Kurchatov Institute", PNPI, St. Petersburg, Russia

${ }^{135}$ Department of Physics and Astronomy, University of Pittsburgh, Pittsburgh Pennsylvania, USA 
${ }^{136 a}$ Laboratório de Instrumentação e Física Experimental de Partículas-LIP, Lisboa, Portugal

${ }^{136 \mathrm{~b}}$ Departamento de Física, Faculdade de Ciências, Universidade de Lisboa, Lisboa, Portugal

${ }^{136 c}$ Departamento de Física, Universidade de Coimbra, Coimbra, Portugal

${ }^{136 \mathrm{~d}}$ Centro de Física Nuclear da Universidade de Lisboa, Lisboa, Portugal

${ }^{136 e}$ Departamento de Física, Universidade do Minho, Braga, Portugal

${ }^{136 f}$ Departamento de Física Teórica y del Cosmos, Universidad de Granada, Granada (Spain), Spain

${ }^{136 \mathrm{~g}}$ Dep Física and CEFITEC of Faculdade de Ciências e Tecnologia, Universidade Nova de Lisboa, Caparica, Portugal

${ }^{136 h}$ Instituto Superior Técnico, Universidade de Lisboa, Lisboa, Portugal

${ }^{137}$ Institute of Physics of the Czech Academy of Sciences, Prague, Czech Republic

${ }^{138}$ Czech Technical University in Prague, Prague, Czech Republic

${ }^{139}$ Charles University, Faculty of Mathematics and Physics, Prague, Czech Republic

${ }^{140}$ Particle Physics Department, Rutherford Appleton Laboratory, Didcot, United Kingdom

${ }^{141}$ IRFU, CEA, Université Paris-Saclay, Gif-sur-Yvette, France

${ }^{142}$ Santa Cruz, Institute for Particle Physics, University of California Santa Cruz, Santa Cruz California, USA

${ }^{143 a}$ Departamento de Física, Pontificia Universidad Católica de Chile, Santiago, Chile

${ }^{143 \mathrm{~b}}$ Universidad de la Serena, La Serena, Chile

${ }^{143 c}$ Universidad Andres Bello, Department of Physics, Santiago, Chile

${ }^{143 \mathrm{~d}}$ Instituto de Alta Investigación, Universidad de Tarapacá, Arica, Chile

${ }^{143 e}$ Departamento de Física, Universidad Técnica Federico Santa María, Valparaíso, Chile

${ }^{144}$ Universidade Federal de São João del Rei (UFSJ), São João del Rei, Brazil

${ }^{145}$ Department of Physics, University of Washington, Seattle Washington, USA

${ }^{146}$ Department of Physics and Astronomy, University of Sheffield, Sheffield, United Kingdom

${ }^{147}$ Department of Physics, Shinshu University, Nagano, Japan

${ }^{148}$ Department Physik, Universität Siegen, Siegen, Germany

${ }^{149}$ Department of Physics, Simon Fraser University, Burnaby BC, Canada

${ }^{150}$ SLAC National Accelerator Laboratory, Stanford California, USA

${ }^{151}$ Department of Physics, Royal Institute of Technology, Stockholm, Sweden

${ }^{152}$ Departments of Physics and Astronomy, Stony Brook University, Stony Brook New York, USA

${ }^{153}$ Department of Physics and Astronomy, University of Sussex, Brighton, United Kingdom

${ }^{154}$ School of Physics, University of Sydney, Sydney, Australia

${ }^{155}$ Institute of Physics, Academia Sinica, Taipei, Taiwan

${ }^{156 a}$ E. Andronikashvili Institute of Physics, Iv. Javakhishvili Tbilisi State University, Tbilisi, Georgia

${ }^{156 b}$ High Energy Physics Institute, Tbilisi State University, Tbilisi, Georgia

${ }^{157}$ Department of Physics, Technion, Israel Institute of Technology, Haifa, Israel

${ }^{158}$ Raymond and Beverly Sackler School of Physics and Astronomy, Tel Aviv University, Tel Aviv, Israel

${ }^{159}$ Department of Physics, Aristotle University of Thessaloniki, Thessaloniki, Greece

${ }^{160}$ International Center for Elementary Particle Physics and Department of Physics, University of Tokyo, Tokyo, Japan

${ }^{161}$ Department of Physics, Tokyo Institute of Technology, Tokyo, Japan

${ }^{162}$ Tomsk State University, Tomsk, Russia

${ }^{163}$ Department of Physics, University of Toronto, Toronto ON, Canada ${ }^{164 a}$ TRIUMF, Vancouver BC, Canada

${ }^{164 \mathrm{~b}}$ Department of Physics and Astronomy, York University, Toronto ON, Canada

${ }^{165}$ Division of Physics and Tomonaga Center for the History of the Universe, Faculty of Pure and Applied Sciences, University of Tsukuba, Tsukuba, Japan

${ }^{166}$ Department of Physics and Astronomy, Tufts University, Medford Massachusetts, USA

${ }^{167}$ Department of Physics and Astronomy, University of California Irvine, Irvine California, USA

${ }^{168}$ Department of Physics and Astronomy, University of Uppsala, Uppsala, Sweden

${ }^{169}$ Department of Physics, University of Illinois, Urbana Illinois, USA

${ }^{170}$ Instituto de Física Corpuscular (IFIC), Centro Mixto Universidad de Valencia-CSIC, Valencia, Spain

${ }^{171}$ Department of Physics, University of British Columbia, Vancouver BC, Canada

${ }^{172}$ Department of Physics and Astronomy, University of Victoria, Victoria BC, Canada

${ }^{173}$ Fakultät für Physik und Astronomie, Julius-Maximilians-Universität Würzburg, Würzburg, Germany

${ }^{174}$ Department of Physics, University of Warwick, Coventry, United Kingdom

${ }^{175}$ Waseda University, Tokyo, Japan

${ }^{176}$ Department of Particle Physics and Astrophysics, Weizmann Institute of Science, Rehovot, Israel

${ }^{177}$ Department of Physics, University of Wisconsin, Madison Wisconsin, USA 
${ }^{178}$ Fakultät für Mathematik und Naturwissenschaften, Fachgruppe Physik,
Bergische Universität Wuppertal, Wuppertal, Germany
${ }^{179}$ Department of Physics, Yale University, New Haven Connecticut, USA

${ }^{\mathrm{a}}$ Deceased.

${ }^{\mathrm{b}}$ Also at Department of Physics, King's College London, London, United Kingdom.

${ }^{\mathrm{c}}$ Also at Istanbul University, Dept. of Physics, Istanbul, Turkey.

${ }^{\mathrm{d}}$ Also at Instituto de Fisica Teorica, IFT-UAM/CSIC, Madrid, Spain.

${ }^{\mathrm{e}}$ Also at TRIUMF, Vancouver BC, Canada.

${ }^{\mathrm{f}}$ Also at Department of Physics, University of Fribourg, Fribourg, Switzerland.

${ }^{g}$ Also at Department of Physics and Astronomy, University of Louisville, Louisville, Kentucky, USA.

${ }^{\mathrm{h}}$ Also at Departament de Fisica de la Universitat Autonoma de Barcelona, Barcelona, Spain.

${ }^{\mathrm{i}}$ Also at Moscow Institute of Physics and Technology State University, Dolgoprudny, Russia.

${ }_{\text {j}}$ Also at Faculty of Physics, Sofia University, 'St. Kliment Ohridski', Sofia, Bulgaria.

${ }^{\mathrm{k}}$ Also at Department of Physics, Ben Gurion University of the Negev, Beer Sheva, Israel.

${ }^{1}$ Also at Universita di Napoli Parthenope, Napoli, Italy.

${ }^{\mathrm{m}}$ Also at Institute of Particle Physics (IPP), Canada.

${ }^{\mathrm{n}}$ Also at Bruno Kessler Foundation, Trento, Italy.

${ }^{\circ}$ Also at Department of Physics, St. Petersburg State Polytechnical University, St. Petersburg, Russia.

${ }^{\mathrm{p}}$ Also at Borough of Manhattan Community College, City University of New York, New York New York, USA.

${ }^{\mathrm{q}}$ Also at Department of Physics, California State University, Fresno, USA.

${ }^{\mathrm{r}}$ Also at Department of Financial and Management Engineering, University of the Aegean, Chios, Greece.

${ }^{\mathrm{s}}$ Also at Centro Studi e Ricerche Enrico Fermi, Italy.

${ }^{t}$ Also at Department of Physics, California State University, East Bay, Hayward, California, USA.

${ }^{u}$ Also at Institucio Catalana de Recerca i Estudis Avancats, ICREA, Barcelona, Spain.

${ }^{v}$ Also at Graduate School of Science, Osaka University, Osaka, Japan.

${ }^{\mathrm{w}}$ Also at Physikalisches Institut, Albert-Ludwigs-Universität Freiburg, Freiburg, Germany.

${ }^{\mathrm{x}}$ Also at University of Chinese Academy of Sciences (UCAS), Beijing, China.

${ }^{y}$ Also at Institute of Physics, Azerbaijan Academy of Sciences, Baku, Azerbaijan.

${ }^{\mathrm{z}}$ Also at Yeditepe University, Physics Department, Istanbul, Turkey.

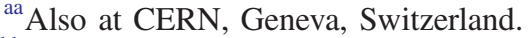

${ }^{\mathrm{bb}}$ Also at Joint Institute for Nuclear Research, Dubna, Russia.

${ }^{c c}$ Also at Hellenic Open University, Patras, Greece.

${ }^{\mathrm{dd}}$ Also at Center for High Energy Physics, Peking University, China.

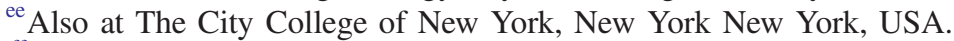

${ }^{\mathrm{ff}}$ Also at Department of Physics, California State University, Sacramento, USA.

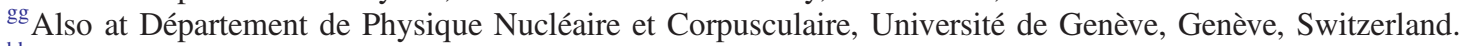

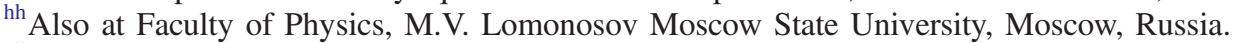

${ }^{\text {ii } A l s o ~ a t ~ I n s t i t u t ~ f u ̈ r ~ E x p e r i m e n t a l p h y s i k, ~ U n i v e r s i t a ̈ t ~ H a m b u r g, ~ H a m b u r g, ~ G e r m a n y . ~}$

${ }^{\mathrm{jj}}$ Also at CPPM, Aix-Marseille Université, CNRS/IN2P3, Marseille, France.

${ }^{\mathrm{kk}}$ Also at National Research Nuclear University MEPhI, Moscow, Russia.

${ }^{11}$ Also at Institute for Particle and Nuclear Physics, Wigner Research Centre for Physics, Budapest, Hungary.

${ }^{\mathrm{mm}}$ Also at Giresun University, Faculty of Engineering, Giresun, Turkey.

${ }^{\mathrm{nn}}$ Also at Department of Physics and Astronomy, Michigan State University, East Lansing Michigan, USA. 